\title{
ENDOMORPHISM ALGEBRAS OF MOTIVES ATTACHED TO ELLIPTIC MODULAR FORMS
}

\author{
ALEXANDER F. BROWN AND EKNATH P. GHATE
}

\begin{abstract}
We study the endomorphism algebra of the motive attached to a non-CM elliptic modular cusp form. We prove that this algebra has a subalgebra isomorphic to a certain crossed product algebra $X$. The Tate conjecture predicts that $X$ is the full endomorphism algebra of the motive. We also investigate the Brauer class of $X$. For example we show that if the nebentypus is real and $p$ is a prime that does not divide the level, then the local behaviour of $X$ at a place lying above $p$ is essentially determined by the corresponding valuation of the $p$-th Fourier coefficient of the form.
\end{abstract}

\section{Contents}

1. Introduction

2. Endomorphism algebras of modular motives 6

2.1. Motives

2.2. Modular motives

2.3. Crossed product structure 10

2.4. Tate conjecture for $M_{f} \quad 27$

2.5. The Brauer class of $X \quad 27$

3. Local behaviour of $X \quad 29$

3.1. Infinite places 29

3.2. Modular crystals 29

3.3. Good places 31

3.4. Bad places 34

4. The case of quadratic twisting 36

4.1. Symbols 37

4.2. The case $a_{p}=0 \quad 43$

4.3. Numerical examples 44

References $\quad 45$

\section{INTRODUCTION}

In this paper we study the endomorphism algebras of motives attached to elliptic modular cusp forms.

2000 Mathematics Subject Classification. Primary: 11G18.

Key words and phrases. Endomorphism algebras, modular motives, Tate conjecture, filtered $(\phi, N)$-modules, Newton polygons, symbols. 
Let $f=\sum a_{n} q^{n}$ be a primitive cusp form of weight $k \geq 2$, level $N \geq 1$ and nebentypus $\epsilon$. Here primitive means that $f$ is a normalized newform that is a common eigenform of all the Hecke operators of level $N$.

When $k>2$ let us denote by $M_{f}$ the Grothendieck motive attached to $f$ constructed by Scholl in [Sch90]. It is a pure motive defined over $\mathbb{Q}$ of rank 2 and weight $k-1$. Let $\operatorname{End}\left(M_{f}\right)$ denote the endomorphisms of $M_{f}$ which are defined over $\overline{\mathbb{Q}}$ and which are defined using the 'cohomological equivalence' equivalence relation. Set $X_{f}:=\operatorname{End}\left(M_{f}\right) \otimes \mathbb{Q}$.

Our first result concerns the structure of $X_{f}$. To state it we need some notation. Assume that $k \geq 2$. Let $E=\mathbb{Q}\left(a_{n}\right)$ denote the Hecke field of $f$. It is well known that $E$ is either a totally real or a CM number field. Now assume that $f$ does not have complex multiplication. A pair $\left(\gamma, \chi_{\gamma}\right)$ where $\gamma \in \operatorname{Aut}(E)$ and $\chi_{\gamma}$ is an $E$-valued Dirichlet character is said to be an extra twist for $f$ if $a_{p}^{\gamma}=a_{p} \cdot \chi_{\gamma}(p)$ for all but finitely many primes $p$. Since $f$ does not have complex multiplication the primitive character associated to $\chi_{\gamma}$ is uniquely determined by $\gamma$. Let $\Gamma$ denote the set of $\gamma \in \operatorname{Aut}(E)$ such that $f$ has a twist by $\left(\gamma, \chi_{\gamma}\right)$ for some $E$-valued Dirichlet character $\chi_{\gamma}$. It turns out that $\Gamma$ is an abelian subgroup of $\operatorname{Aut}(E)$.

For a Dirichlet character $\chi$ let $G(\chi)=\sum_{a=1}^{r} \chi_{0}(a) e^{2 \pi i a / r}$ denote the Gauss sum of the primitive Dirichlet character $\chi_{0}$, of conductor say $r$, associated to $\chi$. For $\gamma$, $\delta \in \Gamma$ set

$$
c(\gamma, \delta)=\frac{G\left(\chi_{\gamma}^{-1}\right) G\left(\chi_{\delta}^{-\gamma}\right)}{G\left(\chi_{\gamma \delta}^{-1}\right)}
$$

where $\chi_{\delta}^{-\gamma}:=\left(\chi_{\delta}^{-1}\right)^{\gamma}$. Then $c \in \mathrm{Z}^{2}\left(\Gamma, E^{\times}\right)$is a 2-cocycle which turns out to be $E^{\times}$-valued. Let $X$ denote the associated crossed product algebra defined as follows. For each $\gamma \in \Gamma$ let $x_{\gamma}$ denote a formal symbol. Then as an $E$-vector space $X$ is finite dimensional with basis the symbols $x_{\gamma}$

$$
X=\bigoplus_{\gamma \in \Gamma} E x_{\gamma}
$$

and has algebra structure given by the relations

$$
\begin{aligned}
x_{\gamma} \cdot e & =\gamma(e) x_{\gamma} \\
x_{\gamma} \cdot x_{\delta} & =c(\gamma, \delta) x_{\gamma \delta},
\end{aligned}
$$

where $e \in E$ and $\gamma, \delta \in \Gamma$. The first result of this paper is the following theorem (Theorem 2.3.8 in the text).

Theorem 1.0.1. If $f$ does not have complex multiplication and has weight $k>2$ then $X_{f}$ contains an algebra isomorphic to $X$.

Let us recall some history. Assume now that $k=2$. Then Shimura has constructed an abelian variety $A_{f}$ defined over $\mathbb{Q}$ associated to $f$. It is a quotient of the Jacobian of the modular curve over $\mathbb{Q}$ which parameterizes elliptic curves with a point of order $N$. Let $X_{f}=\operatorname{End}\left(A_{f}\right) \otimes \mathbb{Q}$ where $\operatorname{End}\left(A_{f}\right)$ denotes the endomorphisms of $A_{f}$ defined over $\overline{\mathbb{Q}}$. Then, if $f$ does not have complex multiplication, Ribet [Rib80] and Momose [Mom81] have shown that $X_{f}$ is isomorphic to the crossed product algebra $X$. In fact it was the original work of Ribet and Momose in the weight two case that has inspired us to investigate the situation in higher weights. We remark here that the paper [Mom81] also concerns the case $k>2$ but as it was written before [Sch90] it does not explicitly discuss the endomorphism 
algebra of the motive $M_{f}$. Instead in [Mom81] Momose shows that $X$ is isomorphic to a sub-algebra of the endomorphism algebra of the (Betti) realization of $M_{f}$.

Here are a few words about the proof of Theorem 1.0.1. Of key importance are the twisting operators studied by Shimura in [Shi73]. There are exactly as many such operators as there are extra twists $\left(\gamma, \chi_{\gamma}\right)$ for $f$. The main thrust of the proof is to show that each of these twisting operators, which lives a priori in the world of linear algebra, actually arises as a realization of an endomorphism of $M_{f}$. We then show that the finite set of endomorphisms of $M_{f}$ one so obtains, along with the Hecke operators, generates a sub-algebra of $X_{f}$ isomorphic to $X$.

The proof of Theorem 1.0.1 follows the strategy used in the weight two abelian variety case by Ribet in [Rib80]. As in that paper it seems necessary to introduce an auxiliary eigenform $g$ of level $N^{2}$ which is constructed from $f$ by stripping away all the Fourier coefficients which meet $N$. We prove that the endomorphism algebra of $M_{g}$, the motive attached to $g$, contains the crossed product algebra $X$. When $a_{p}=0$ for each $p$ dividing $N$ then $f=g$ and Theorem 1.0.1 follows in this case. When $f \neq g$ we show $M_{g}$ and $M_{f}$ still have isomorphic endomorphism algebras (see Proposition 2.3.9) thereby proving Theorem 1.0.1 in general. The proof of Theorem 1.0.1 occupies most of Section 2.

Shimura has shown [Shi71] that if $f$ has complex multiplication and $k=2$ then the abelian variety $A_{f}$ is isogenous to a self-product of an elliptic curve with complex multiplication by an imaginary quadratic field, say $L$. It follows that in this case $X_{f}$ is isomorphic to a matrix algebra over $L$. We have not investigated the structure of $X_{f}$ when $f$ has complex multiplication and $k>2$.

When $f$ does not have complex multiplication and $k>2$, the question as to whether $X_{f}$ is isomorphic to $X$ is closely related to the Tate conjecture for the motive $M_{f}$. To explain this more precisely we need some notation. Let $\ell$ be a fixed prime and let $M_{\ell}$ denote the $\ell$-adic realization of $M_{f}$. It is a free $E \otimes \mathbb{Q}_{\ell}$ module of rank 2 equipped with an action of the absolute Galois group of $\mathbb{Q}$. For an arbitrary number field $K$ let us denote by $G_{K}$ the absolute Galois group of $K$. Then there is a natural map

$$
\alpha_{K}: \operatorname{End}_{K}\left(M_{f}\right) \otimes \mathbb{Q}_{\ell} \rightarrow \operatorname{End}_{G_{K}}\left(M_{\ell}\right)
$$

where the subscript $K$ on the left of the arrow means that we only consider those endomorphisms that are defined over $K$ and the subscript $G_{K}$ on the right is meant to denote those endomorphisms of the $\mathbb{Q}_{\ell}$-vector space $M_{\ell}$ that commute with the action of $G_{K}$. The Tate conjecture for $M_{f}$ is the statement that $\alpha_{K}$ is an isomorphism for every number field $K$. We prove this subject to an injectivity constraint (see Corollary 2.4.2 in the text).

Corollary 1.0.2 (Tate conjecture). Assume that $\alpha_{K}$ is injective for some suffciently large number field $K$. Then $\alpha_{K}$ is an isomorphism for all number fields $K$. Moreover, in this case, $X_{f}$ is isomorphic to $X$.

The Tate conjecture for the abelian variety $A_{f}$ was proved by Ribet when $k=2$ and $f$ does not have complex multiplication in [Rib80]. As Ribet points out there, if $f$ has complex multiplication and $k=2$ then the Tate conjecture for $A_{f}$ follows from the result of Shimura alluded to above. It is now known that the Tate conjecture holds for any abelian variety defined over a number field by the work of Faltings [Fal83]. 
From now on we will think of the abelian variety $A_{f}$ as the motive attached to $f$ in the case $k=2$. Thus we set $M_{f}=A_{f}$ in this case. Then $X_{f}$ denotes the endomorphism algebra of the motive attached to $f$ in all weights $k \geq 2$. We shall also assume from now on that $f$ does not have complex multiplication.

Let $F$ be the number field contained in $E$ which is the fixed field of $\Gamma$. Then $X$ is a central simple algebra over $F$ and is easily seen to be 2 -torsion when considered as an element of the Brauer group of $F$. As a result $X$ is either a matrix algebra over $F$ or a matrix algebra over a quaternion division algebra over $F$. Ribet has remarked that it seems difficult to distinguish these cases by pure thought. Ribet's remark pertains to the case $k=2$ (in which case $X$ is isomorphic to $X_{f}$ ) but after our work in higher weight it is also relevant when $k \geq 2$.

One of the chief motivations for writing this paper was to prove new results that allow us to distinguish these cases. Our initial goal was to generalize Ribet's weight 2 results in [Rib81] concerning the structure of $X$ to higher weight. Some of these generalizations turned out to be routine and others have yielded some surprises. On the other hand along the way we were able to prove results that are new even for $k=2$. Here is a more detailed description of these results.

Recall that by global class field theory there is an injection $\operatorname{Br}(F) \hookrightarrow \oplus_{v} \operatorname{Br}\left(F_{v}\right)$ where $v$ runs through the places of $F$ and $F_{v}$ is the completion of $F$ at $v$. Thus to study the Brauer class of $X$ it suffices to study its image $X_{v}=X \otimes_{F} F_{v}$ for each place $v$ of $F$ under the above map. Since $X$ is 2-torsion in the Brauer group of $F$, the algebra $X_{v}$ is a fortiori either a matrix algebra over $F_{v}$ or a matrix algebra over a quaternion division algebra over $F_{v}$.

Momose [Mom81] has already determined the structure of $X_{v}$ when $v$ is an infinite place of $F$. In fact all such places must be real ( $F$ is a totally real number field since if $E$ is a CM field then complex conjugation is always an element of $\Gamma$ ). Momose proves that $X_{v}$ is a matrix algebra over $\mathbb{R}$ if and only if $k$ is even. So let us assume for the rest of the introduction that $v$ is a finite place of $F$, say of residue characteristic $p$. For convenience of exposition we shall say that $v$ is 'good' if $p$ is prime to $N$ and that $v$ is 'bad' otherwise.

Assume that $v$ is good. Then a short computation shows that $a_{p}^{2} \epsilon(p)^{-1} \in F$. Ribet has shown that if $k=2$ then $X_{v}$ is a matrix algebra over $F_{v}$ if $v\left(a_{p}^{2} \epsilon(p)^{-1}\right)=0$. We generalize this as follows (see Theorems 3.3.1 and 3.3.2 in the text).

Theorem 1.0.3. Assume $v$ is good. If $k \geq 2$ then $X_{v}$ is a matrix algebra over $F_{v}$ if

- $0 \leq v\left(a_{p}^{2} \epsilon(p)^{-1}\right)<k-1$, and,

- $w\left(a_{p}\right)$ has odd denominator where $w \mid v$ is any place of $E$.

Note that the second condition is automatically satisfied if $v\left(a_{p}^{2} \epsilon(p)^{-1}\right)=0$, so this is indeed a generalization of Ribet's result. The above theorem yields, at least in principle, new information even when $k=2$.

The proof of Theorem 1.0.3 is based on a study of the filtered $(\phi, N)$-module associated, by the theory of Fontaine, to the restriction to a decomposition group at $p$ of the $w$-adic Galois representations attached to $f$ (see Section 3 ). The underlying principle of the proof is simple and can be stated roughly as follows. Under hypotheses such as the above on the valuations of $a_{p}$ the slopes of the crystalline Frobenius naturally break the filtered $(\phi, N)$-module into two pieces. Since the 
relevant endomorphism algebra must preserve these individual pieces it is forced to split, that is it is forced to be a matrix algebra over $F_{v}$.

A shortcoming of Theorem 1.0.3 is that it does not tell us when $X_{v}$ is not a matrix algebra over $F_{v}$. With this in mind we now restrict our attention to a class of cusp forms $\mathcal{Q}$ whose members have endomorphism algebras that are more amenable to computation (see Section 4 ). We take $\mathcal{Q}$ to be the class of primitive non-CM forms for which all the extra twists $\chi_{\gamma}$ are quadratic characters. It is not hard to see that $\mathcal{Q}$ consists exactly of the primitive non-CM forms whose nebentypus character $\epsilon$ is real.

For $f \in \mathcal{Q}$ we prove a formula which expresses the Brauer class of $X$ in terms of symbols. (See Theorem 4.1.3. After our work was completed, it was pointed out to us that a similar formula was proved earlier by Quer [Que98] in the case $k=2$.) The entries of these symbols are built in a simple way out of the divisors of $N$ and certain Fourier coefficients which are completely determined by the extra twists of $f$. The explicit nature of this formula allows us to determine the structure of $X_{v}$ at all the places of $F$. When $v$ is a good finite place the answer we obtain is surprisingly simple (see Theorem 4.1.11 in the text).

Theorem 1.0.4. Suppose that $f \in \mathcal{Q}$ and that $k \geq 2$. Suppose that $v$ is good. Assume $p \neq 2$. If $a_{p} \neq 0$ then $X_{v}$ is a matrix algebra over $F_{v}$ if and only if the integer

$$
m_{v}:=\left[F_{v}: \mathbb{Q}_{p}\right] \cdot v\left(a_{p}^{2} \epsilon(p)^{-1}\right)
$$

is even, except possibly in the exceptional case that $p$ splits in all the quadratic fields cut out by the level $N$, in which case $X_{v}$ is necessarily a matrix algebra over $F_{v}$.

Roughly speaking Theorem 1.0.4 says that the local behaviour of $X$ at the good $p$-adic places is essentially determined by the parity of the $p$-adic valuations of the $p^{\text {th }}$ Fourier coefficient of $f$ !

For a more precise explanation of the exceptional case in the statement of Theorem 1.0.4 we refer the reader to Theorem 4.1.11 in the text. We remark here that Theorem 1.0.4 is also true when $p=2$ if we assume that $F=\mathbb{Q}$. We have not treated the general case when $p=2$ since we have not yet found a suitable reference for wild symbols for number fields other than $\mathbb{Q}$. On the other hand Theorem 1.0.4 makes no sense if $a_{p}=0$ since in this case $m_{v}$ blows up. Yet it turns out that a similar result also holds even when $a_{p}=0$ : one simply replaces $a_{p}$ in the statement above by any non-zero Fourier coefficient $a_{p^{\dagger}}$ where $p^{\dagger}$ is a prime satisfying $p p^{\dagger} \equiv 1$ $\bmod N$ (see Proposition 4.2.1).

Let us now turn our attention to the bad places $v$ of $F$ (see Section 3.4). Let $N_{p}$ denote the exponent of $p$ dividing the level $N$ and let $C_{p}$ denote the exponent of $p$ dividing the conductor $C$ of $\epsilon$. Note that $N_{p} \geq 1$ by hypothesis and $N_{p} \geq C_{p}$. Our results at the bad places are broken down according to how $N_{p}$ compares with $C_{p}$. This roughly corresponds to how the local factor at $p$ of the automorphic representation attached to $f$ behaves, namely whether it is in the ramified principal series, is Steinberg, is supercuspidal, etc.

To give some examples of our results suppose that $p \| N$ and that $p \mid C$, so that the local automorphic representation at $p$ is in the ramified principal series. We then have the following theorem (see Theorem 3.4.2). 
Theorem 1.0.5. Say that $k \geq 2$. Say that $v$ is bad with $N_{p}=C_{p}=1$. If one of $w\left(a_{p}\right)$ or $w\left(\bar{a}_{p}\right)$ vanishes for each place $w$ of $E$ lying over $v$ then $X_{v}$ is a matrix algebra over $F_{v}$.

On the other hand suppose that $p \| N$ but that $p \nmid C$. Then the local automorphic factor at $p$ is a twist by an unramified character of the Steinberg representation. For brevity we shall say that $f$ is Steinberg at $p$, or that we are in the Steinberg case. In this case we have the following generalization of a result of Ribet in weight two (see Theorem 3.4.6).

Theorem 1.0.6. Suppose that $v$ is bad with $N_{p}=1$ and $C_{p}=0$. If $k \geq 2$ is even then $X_{v}$ is a matrix algebra over $F_{v}$. In fact $X$ is a matrix algebra over $F$.

We give an example which shows that it is possible for $X_{v}$ to be ramified when $k$ is odd. Thus the naive generalization to higher weight of Ribet's weight two theorem in the Steinberg case is false! However we show (see Theorem 3.4.8) that when $k$ is odd then $X$ is unramified everywhere except possibly at the places of $F$ lying above $p$ and $\infty$. An amusing corollary of this is that if $k$ is odd and $f$ is Steinberg at at least two primes then $X$ is ramified exactly at infinity (see Corollary 3.4.9).

An important part of the proofs of Theorems 1.0.5 and 1.0.6 is based, like the proof of Theorem 1.0.3, on an analysis of the slopes of the filtered $(\phi, N)$-modules attached to the local Galois representations in the above cases. These filtered modules have been written down explicitly by Breuil [Bre01] in the good cases $(p \nmid N)$, and in some bad cases $(p \mid N)$. In this context see also the original work of Fontaine and Mazur [FM83], and of Volkov [Vol01] (for elliptic curves). It seems desirable therefore to have a complete classification of the filtered $(\phi, N)$-modules attached to primitive cusp forms in all cases. This problem was posed by Breuil in [Bre01].

We end the paper with some numerical examples to illustrate the theorems proved in this paper. The computations were done with the help of our program Endohecke which makes extensive use of the modular symbols engine from W. Stein's C++ program Hecke. More detailed output from this program may appear elsewhere.

\section{ENDOMORPHism ALGEBRAS OF MODUlAR MOTIVES}

Let us keep the notation of the introduction. In particular $f$ is a primitive form without complex multiplication of weight $k \geq 2, M_{f}$ is the motive attached to $f$, $X_{f}$ is the $\mathbb{Q}$-algebra of endomorphisms of $M_{f}, E$ is the number field generated by the Fourier coefficients $a_{n}$ of $f$, and $F$ is the subfield of $E$ fixed by $\Gamma$.

In this section we show that $X_{f}$ contains a sub-algebra which is isomorphic to the crossed product algebra $X$. We explain how this statement is connected to the Tate conjecture for the motive $M_{f}$. We show $X$ is a central simple algebra over $F$ which is 2-torsion in the Brauer group of $F$. We prove that $X$ is trivial in the Brauer group of $F$ if all the elements of $X$ are defined over the real numbers.

2.1. Motives. For the convenience of the reader we start by recalling some of the basic definitions and properties of Grothendieck motives. We follow the introduction of [BR93, Section 1].

Let $K \subset \mathbb{C}$ be a number field equipped with a fixed embedding into $\mathbb{C}$. Let $X$ and $Y$ be smooth projective varieties defined over $K$ and suppose that each 
geometrically irreducible component of $X$ has dimension $d$. Let $Z(X \times Y)$ be the rational vector space generated by the irreducible sub-varieties of $X \times Y$, defined over $K$, of pure codimension $d$. Let $Z_{h}(X \times Y)$ be the quotient

$$
Z_{h}(X \times Y):=Z(X \times Y) / \sim
$$

where $\sim$ is the cohomological equivalence relation; i.e. for any $Z \in Z_{h}(X \times Y)$, $Z \sim 0$ if and only if the image of $Z$ in $\mathrm{H}_{B}^{2 d}(X \times Y)(d)$ under the cycle class map is zero. Endow $Z_{h}(X \times Y)$ with the multiplication operation given by composition product of correspondences.

An effective motive is a pair $M=(X, p)$ where $p \in Z_{h}(X \times X)$ is a projector, that is it satisfies $p^{2}=p$. If $N=(Y, q)$ is another effective motive then $\operatorname{Hom}(M, N)$ is defined to be

$$
\operatorname{Hom}(M, N):=\frac{\left\{Z \in Z_{h}(X \times Y): Z \circ p=q \circ Z\right\}}{\left\{Z \in Z_{h}(X \times Y): Z \circ p=q \circ Z=0\right\}} .
$$

The category of effective motives over $K$ thus defined has a tensor structure given by

$$
M \otimes N:=(X \times Y, p \times q) .
$$

Let $L$ be the effective motive $\left(\mathbb{P}^{1}, 1-Z\right)$ where $Z \in Z_{h}\left(\mathbb{P}^{1} \times \mathbb{P}^{1}\right)$ is the class of the cycle $\mathbb{P}^{1} \times\{$ point $\}$. The functor sending $M$ to $M \otimes L$ is fully faithful, so that it can be formally inverted.

Definition 2.1.1. A motive is a pair $(M, a)$, where $M$ is an effective motive and $a \in \mathbb{Z}$. $(M, a)$, also denoted $M(a)$, is the $a$-fold Tate twist of $M$. If $N(b)$ is another motive then $\operatorname{Hom}(M(a), N(b))$ is defined as

$$
\operatorname{Hom}(M(a), N(b)):=\operatorname{Hom}\left(M \otimes L^{r-a}, N \otimes L^{r-b}\right)
$$

for any $r \geq \max \{a, b\}$.

This defines the category of Grothendieck motives over $K$. In this category projectors have kernels and images. However it is not known whether this category is abelian. If however one uses numerical equivalence instead of cohomological equivalence to define morphisms then the resulting category is known to be abelian and semi-simple [Jan92].

The effective motive $M=(X, p)$ has a realization in each of the standard cohomology theories. For instance there is the Betti realization

$$
M_{B}=\operatorname{Im}\left(p_{B}: \mathrm{H}_{B}^{*}(X) \rightarrow \mathrm{H}_{B}^{*}(X)\right)
$$

of $M$, which depends on the choice of embedding $K \subset \mathbb{C}$. Similarly, for each prime $\ell$ there is the $\ell$-adic realization

$$
M_{\ell}=\operatorname{Im}\left(p_{\ell}: \mathrm{H}_{\ell}^{*}(X) \rightarrow \mathrm{H}_{\ell}^{*}(X)\right)
$$

of $M$, which comes equipped with an action of $\operatorname{Gal}(\overline{\mathbb{Q}} / \mathbb{Q})$. The realizations of the motive $M(a)$ for $a \in \mathbb{Z}$ are easily described in terms of the those of the effective motive $M: M(a)_{B}=M_{B} \otimes(2 \pi i)^{a} \mathbb{Q}$ and $M(a)_{\ell}=M_{\ell} \otimes \chi_{\ell}^{a}$ where $\chi_{\ell}$ is the $\ell$-th cyclotomic character. 
2.2. Modular motives. Let $f$ be a primitive elliptic modular cusp form. In this section we recall the definition of the motive $M_{f}$ attached to $f$. When $f$ has weight 2 then $M_{f}$ is well understood: in this case it is an abelian variety. So we shall assume that $f$ has weight larger than 2 .

Write $k+2$ for the weight of $f$ and $n=N$ for the level of $f$. This change in notation will be in force for the remainder of this section and is done in order to conform to the notation used by Scholl in [Sch90]. Thus we assume that $k>0$. We shall also assume that $n \geq 3$ although this is not necessary (cf. [Sch90]).

Let $M_{n}$ denote the modular curve over $\mathbb{Q}$ representing the functor which associates to any $\mathbb{Q}$-scheme $S$ the set of isomorphism classes of elliptic curves $E$ over $S$ with level $n$ structure $\sigma: E[n] \stackrel{\sim}{\rightarrow}(\mathbb{Z} / n)^{2}$. Here $E[n]:=\operatorname{ker}([n]: E \rightarrow E)$. Let $j: M_{n} \hookrightarrow \bar{M}_{n}$ denote the smooth compactification of $M_{n}$. Let

$$
\pi: X_{n} \rightarrow M_{n}
$$

denote the universal elliptic curve and let $\bar{\pi}: \bar{X}_{n} \rightarrow \bar{M}_{n}$ denote the universal generalized elliptic curve. These objects exists since $n \geq 3$. Let

$$
\bar{\pi}_{k}: \bar{X}_{n}^{k} \rightarrow \bar{M}_{n}
$$

denote the $k$-fold fiber product of $\bar{X}_{n}$ with itself over $\bar{M}_{n}$ and let $\bar{X}_{n}^{k}$ denote the canonical desingularization of $\bar{X}_{n}^{k}$ constructed by Deligne.

The group $(\mathbb{Z} / n)^{2}$ acts on $\bar{X}_{n}$ by translation in the fibers and $\mu_{2}$ acts on $\bar{X}_{n}$ by inversion in the fibers. This induces an action of the semi-direct product $(\mathbb{Z} / n)^{2} \rtimes \mu_{2}$ on $\bar{X}_{n}$. Let $\Sigma_{k}$ be the symmetric group on $k$ letters. Then the group

$$
\Gamma_{k}=\left((\mathbb{Z} / n)^{2} \rtimes \mu_{2}\right)^{k} \rtimes \Sigma_{k}
$$

acts on $\bar{X}_{n}^{k}$. By the canonical nature of the desingularization this action extends to an action of $\Gamma_{k}$ on $\overline{\bar{X}}_{n}^{k}$.

Let $\varepsilon: \Gamma_{k} \rightarrow\{ \pm 1\}$ denote the homomorphism which is trivial on $(\mathbb{Z} / n)^{2 k}$, is the product map on $\mu_{2}^{k}$ and is the sign character on $\Sigma_{k}$. Let

$$
\Pi_{\varepsilon}=\frac{1}{\left|\Gamma_{k}\right|} \sum_{g \in \Gamma_{k}} \varepsilon(g) \cdot g \quad \in \mathbb{Z}[1 /(2 n \cdot k !)]\left[\Gamma_{k}\right]
$$

denote the projector attached to $\varepsilon$ and let $W_{n}=\left(\overline{\bar{X}}_{n}^{k}, \Pi_{\varepsilon}\right)$ be the associated motive.

The group $\mathrm{GL}_{2}(\mathbb{Z} / n)$ acts on $\bar{X}_{n}$ via

$$
\gamma:(e \in E, \sigma) \mapsto(e \in E, \gamma \circ \sigma),
$$

where $\gamma \in \mathrm{GL}_{2}(\mathbb{Z} / n)$. Here we allow $E$ to be a generalized elliptic curve, or Néron $n$-gon in the sense of [DR73]. This action extends in the obvious way to an action of $\mathrm{GL}_{2}(\mathbb{Z} / n)$ on $\bar{X}_{n}^{k}$.

Lemma 2.2.2. This action of $\mathrm{GL}_{2}(\mathbb{Z} / n)$ on $\bar{X}_{n}^{k}$ is $\Pi_{\varepsilon}$-equivariant.

Proof. Consider first the actions of $\mathrm{GL}_{2}(\mathbb{Z} / n)$ and $(\mathbb{Z} / n)^{2}$ on $\bar{X}_{n}$. For any point $(e \in E, \sigma)$ of $\bar{X}_{n}$ and $\gamma \in \mathrm{GL}_{2}(\mathbb{Z} / n)$ the correspondence $\left(\sum_{g \in(\mathbb{Z} / n)^{2}} g\right) \circ \gamma$ applied to $(e, \sigma)$ gives the formal sum

$$
\sum_{g \in(\mathbb{Z} / n)^{2}}\left(e+(\gamma \circ \sigma)^{-1}(g), \gamma \circ \sigma\right) .
$$


On the other hand $\gamma \circ \sum_{g \in(\mathbb{Z} / n)^{2}} g$ applied to $(e, \sigma)$ produces

$$
\sum_{g \in(\mathbb{Z} / n)^{2}}\left(e+\sigma^{-1}(g), \gamma \circ \sigma\right) .
$$

As these two formal sums are equal the correspondence on $\bar{X}_{n}$, and hence on $\bar{X}_{n}^{k}$, given by $\sum_{g \in(\mathbb{Z} / n)^{2}} g$ commutes with $\gamma$. Since the action of $\gamma$ on $\bar{X}_{n}^{k}$ commutes with the action of $\mu^{2 k}$ and $\Sigma_{k}$ we see that $\gamma$ commutes with the action of $\Pi_{\varepsilon}$ as well.

The (left) action of $\mathrm{GL}_{2}(\mathbb{Z} / n)$ on $\bar{X}_{n}^{k}$ extends to an action on $\overline{\bar{X}}_{n}^{k}$ which by the lemma above is $\Pi_{\varepsilon}$-equivariant and hence gives a (right) action of $\mathrm{GL}_{2}(\mathbb{Z} / n)$ on $W_{n}$. Let

$$
\Gamma_{n}=\left\{\left(\begin{array}{ll}
* & 0 \\
0 & 1
\end{array}\right) \in \mathrm{GL}_{2}(\mathbb{Z} / n)\right\} .
$$

The notation should not cause any confusion with the group denoted by $\Gamma_{k}$ above. Let $\Gamma_{n}^{1}$ denote the subgroup of $\mathrm{GL}_{2}(\mathbb{Z} / n)$ of matrices of the form $\left(\begin{array}{c}* * * \\ 0\end{array}\right)$. Let $I_{f}$ denote the annhilator of $f$ in the $\Gamma_{1}(n)$ Hecke algebra. Scholl defines $M_{f}$ to be the sub-motive of $W_{n}^{\Gamma_{n}^{1}} \subset W_{n}^{\Gamma_{n}}$ which is the kernel of $I_{f}$ acting on $W_{n}^{\Gamma_{n}^{1}}$. The motive $M_{f}$ is defined over $\mathbb{Q}$, has rank 2 , weight $k+1$ and, via the action of the Hecke operators, coefficients in $E$.

In this paper we will be interested in studying the endomorphisms of $M_{f}$ which are defined over $\overline{\mathbb{Q}}$. To this end set $X_{f}=\operatorname{End}\left(M_{f}\right) \otimes \mathbb{Q}$, where $\operatorname{End}\left(M_{f}\right)$ denotes the endomorphisms of $M_{f}$ which are defined over arbitrary number fields. Note here that tensoring by $\mathbb{Q}$ in the definition of $X_{f}$ is actually redundant in view of the definitions made in Section 2.1, but we write it anyway since in the abelian variety situation it is really the algebra $X_{f}=\operatorname{End}\left(A_{f}\right) \otimes \mathbb{Q}$ and not $\operatorname{End}\left(A_{f}\right)$ which was studied by Momose and Ribet.

In the next section we will find it easier as to work as in [Rib80] with an auxiliary modular form $g$ which is constructed from $f$ as follows:

$$
g=\sum_{(m, n)=1} a_{m} q^{m} .
$$

Thus the $q$-expansion of $g$ is obtained from that of $f$ by stripping all the Fourier coefficients of $f$ which meet the level $n$. A short computation shows that

$$
g(z)=\sum_{d \mid n} \mu(d) \cdot a_{d} \cdot f(d z)
$$

where $\mu$ denotes the Möbius function. The relation (2.2.3) shows that $g$ is in fact a cusp form, and that it satisfies the usual automorphy property with respect to elements in $\Gamma_{1}\left(n^{2}\right)$. In fact if $\Gamma_{2}(n) \supset \Gamma_{1}\left(n^{2}\right)$ denotes the congruence subgroup defined by

$$
\Gamma_{2}(n)=\left\{\left(\begin{array}{ll}
a & b \\
c & d
\end{array}\right) \in \mathrm{SL}_{2}(\mathbb{Z}) \mid a \equiv d \equiv 1 \bmod n, c \equiv 0 \bmod n^{2}\right\}
$$

then $g \in S_{k}\left(\Gamma_{2}(n)\right) \subset S_{k}\left(\Gamma_{1}\left(n^{2}\right)\right)$ since $f(d z) \in S_{k}\left(\Gamma_{2}(n)\right)$ for each $d \mid n$. It is also easily checked that $g$ is an eigenform of all the Hecke operators of level $n^{2}$. 
One may attach a motive $M_{g}$ to $g$ mimicking Scholl's construction. One simply replaces $n$ by $n^{2}$ to obtain the motive $W_{n^{2}}$ as above. Let $I_{g}$ denote the annhilator of $g$ in the $\Gamma_{1}\left(n^{2}\right)$ Hecke algebra. Let

$$
\Gamma_{n}^{2}=\left\{\left(\begin{array}{cc}
* & * \\
0 & 1(n)
\end{array}\right) \in \mathrm{GL}_{2}\left(\mathbb{Z} / n^{2}\right)\right\}
$$

where $1(n)$ means that the $(2,2)$ entry satisfies $x \equiv 1 \bmod n$. We define $M_{g}$ to be the sub-motive of $W_{n^{2}}^{\Gamma_{n}^{2}} \subset W_{n^{2}}^{\Gamma_{n^{2}}}$ which is the kernel of $I_{g}$ acting on $W_{n^{2}}^{\Gamma_{n}^{2}}$. Then the motive $M_{g}$, like $M_{f}$, is defined over $\mathbb{Q}$, has rank 2 , weight $k+1$ and coefficients in E.

2.3. Crossed product structure. In this section we will show that $X_{f}$, the $\mathbb{Q}$ algebra of endomorphisms of $M_{f}$, contains a sub-algebra isomorphic to the crossed product algebra $X$.

Let $\gamma \in \operatorname{Aut}(E)$ and let $\chi$ be a Dirichlet character that takes values in $E^{\times}$. We say that $f$ has an extra twist or simply a twist by $(\gamma, \chi)$ if

$$
a_{p}^{\gamma}=\chi_{\gamma}(p) \cdot a_{p}
$$

for all but finitely many primes $p$. Ribet calls such a phenomenon an inner twist in [Rib80] but we prefer to use the above terminology. When $\epsilon$ is non-trivial and $f$ does not have complex multiplication the prototype of an extra twist for $f$ is $\left(c, \epsilon^{-1}\right)$ where $c$ denotes complex conjugation on the CM field $E$.

The reader might envision an apparently more general definition of an extra twist wherein $\gamma$ is an embedding of $E$ into $\overline{\mathbb{Q}}$ and $\chi$ is a $\overline{\mathbb{Q}}$-valued Dirichlet character. However it turns out that both $\gamma$ and $\chi$ are then necessarily $E$-valued. Indeed by comparing determinants of the Galois representations associated to $f$ and $f^{\gamma}$ one sees that $\epsilon^{\gamma-1}=\chi^{2}$. Let $m$ be the order of $\epsilon$. If $m$ is even then the order of $\epsilon^{\gamma-1}$ divides $m / 2$ so that the order of $\chi$ divides $m$. On the other hand if $m$ is odd then the order of $\epsilon^{\gamma-1}$ divides $m$ so that the order of $\chi$ divides $2 m$. Since $\mathbb{Q}\left(\mu_{2 m}\right)=\mathbb{Q}\left(\mu_{m}\right)$ if $m$ is odd we see that in either case $\mathbb{Q}(\chi) \subset \mathbb{Q}\left(\mu_{m}\right)=\mathbb{Q}(\epsilon)$. But it is well known that $\mathbb{Q}(\epsilon) \subset E$. Thus $\chi$ is $E$-valued. The relation (2.3.1) along with the fact that the $a_{p}$ 's generate $E$ show that $\gamma$ is also $E$-valued.

If $f$ has a twist by $(\gamma, \chi)$ then the primitive Dirichlet character associated to $\chi$ is uniquely determined by $\gamma$ since $f$ does not have complex multiplication. We therefore write $\chi_{\gamma}$ for $\chi$.

Let $\Gamma \subset \operatorname{Aut}(E)$ denote the subgroup of $\gamma \in \operatorname{Aut}(E)$ such that $f$ has a twist by $\left(\gamma, \chi_{\gamma}\right)$ for some $E$-valued Dirichlet character $\chi_{\gamma}$. The following observation is due to Ribet [Rib80] (in the weight two case) and will not be used in the sequel.

Lemma 2.3.2. $\Gamma$ is an abelian subgroup of $\operatorname{Aut}(E)$.

Proof. As Momose has observed in [Mom81], for each $\gamma \in \Gamma$ there exists an integer $k$ (depending on $\gamma$ ) so that $\epsilon^{\gamma-1}=\epsilon^{2 k}$; in view of the relation $\chi_{\gamma}^{2}=\epsilon^{\gamma-1}$ each $\chi_{\gamma}$ is the product of a quadratic character and a power of $\epsilon$. A short computation using the identity

$$
\chi_{\gamma \delta}=\chi_{\gamma} \chi_{\delta}^{\gamma}
$$

now shows that the character corresponding to $\gamma \delta$ is the same as the character corresponding to $\delta \gamma$. 
Let $M$ denote the set of $E$-valued Dirichlet characters. By (2.3.3) the map

$$
\gamma \mapsto \chi_{\gamma}
$$

defines a cocycle $c^{\prime}: \Gamma \rightarrow M$ and therefore determines a cohomology class in $\mathrm{H}^{1}(\Gamma, M)$. The relation $\chi_{\gamma}^{2}=\epsilon^{\gamma-1}$ shows that this class is 2-torsion.

Consider the 2-cocycle $c \in \mathrm{Z}^{2}\left(\Gamma, E^{\times}\right)$defined by

$$
c(\gamma, \delta)=\frac{G\left(\chi_{\gamma}^{-1}\right) G\left(\chi_{\delta}^{-\gamma}\right)}{G\left(\chi_{\gamma \delta}^{-1}\right)}
$$

where $G(\chi)$ is the Gauss sum of the primitive Dirichlet character associated to $\chi$. The relation (2.3.3) shows that $c(\gamma, \delta)$ is a actually a Jacobi sum. That $c$ is $E$-valued follows from a standard formula for the action of Jacobi sums under $\operatorname{Gal}(\overline{\mathbb{Q}} / E)$ along with the fact that the characters involved in the definition of $c(\gamma, \delta)$ are all $E$-valued. Let $X$ be the central simple algebra attached to $c$ defined as follows. For each $\gamma \in \Gamma$ let $x_{\gamma}$ denote a formal symbol. Consider the $E$-vector space $X$ with basis the symbols $x_{\gamma}$ :

$$
X=\bigoplus_{\gamma \in \Gamma} E x_{\gamma}
$$

Let $F$ denote the fixed field of $\Gamma$. Make $X$ into an $F$-algebra via the relations

$$
\begin{aligned}
x_{\gamma} \cdot e & =\gamma(e) x_{\gamma} \\
x_{\gamma} \cdot x_{\delta} & =c(\gamma, \delta) x_{\gamma \delta}
\end{aligned}
$$

where $e \in E$ and $\gamma, \delta \in \Gamma$. Clearly $F$ is the center of $X$. The following proposition, which was observed by Serre in the case $k=2$ (cf. [Rib80, page 49]), gives some more information about $F$.

Proposition 2.3.5. $F$ is the subfield of $E$ generated by the elements $a_{p}^{2} \epsilon(p)^{-1}$ as $p$ varies through the prime numbers such that $p \nmid N$.

Proof. Let $\left(\gamma, \chi_{\gamma}\right)$ be an extra twist for $f$. We claim that $a_{p}^{\gamma}=a_{p} \cdot \chi_{\gamma}(p)$ for all $p \nmid N$. Indeed, since the claim holds for all but finitely many primes $p$, the primitive form associated to the form $\sum a_{n} \cdot \chi_{\gamma}(n) q^{n}$ must, by uniqueness results for newforms, equal the primitive level $N$ form $f^{\gamma}=\sum a_{n}^{\gamma} q^{n}$. It follows that if $r$ is the conductor of $\chi_{\gamma}$ then the claim holds for all primes $p \nmid r N$. But a short computation using [AL78, Theorem 3.1 ii b)], which is concerned with the exact level of twists of primitive forms, shows that $r \mid N$. The claim follows.

To prove the proposition note that the relations $a_{p}^{\gamma}=a_{p} \chi_{\gamma}(p)$ and $\chi_{\gamma}^{2}=\epsilon^{\gamma-1}$ show that each $\gamma \in \Gamma$ fixes $a_{p}^{2} \epsilon(p)^{-1}$ for $p \nmid N$. So $\mathbb{Q}\left(a_{p}^{2} \epsilon(p)^{-1}: p \nmid N\right)$ is contained in the fixed field of $\Gamma$, namely $F$. (This is the only part of the proposition that we need in the sequel). To show that $F$ is in fact generated by these elements we refer the reader to [Rib92, 5.3] where this is proved for $k=2$ and note that Ribet's proof generalizes verbatim to higher weight.

There is a natural homomorphism (cf. [Rib81, Proposition 2])

$$
\mathrm{H}^{1}(\Gamma, M) \longrightarrow \mathrm{H}^{2}\left(\Gamma, E^{\times}\right)
$$

which takes the class of $c^{\prime}$ to the class of $c$. Under the inflation map

$$
\text { Inf : } \mathrm{H}^{2}\left(\Gamma, E^{\times}\right)=\operatorname{Br}(E / F) \hookrightarrow \mathrm{H}^{2}\left(G_{F}, \bar{F}^{\times}\right)=\operatorname{Br}(F),
$$


$X$ may be considered as an element of the Brauer group $\operatorname{Br}(F)$ of $F$. Since the class of $c^{\prime}$ is 2-torsion, the class of $X$ lies in $\operatorname{Br}(F)[2]$.

The importance of the algebra $X$, at least when $k=2$, stems from the following theorem of Ribet [Rib80, Theorem 5.1] and Momose [Mom81, Theorem 4.1].

Theorem 2.3.6. If $f$ is of weight 2 then $X_{f}$ is isomorphic to $X$.

Returning to the general case let $M_{B}$ denote the Betti realization of $M_{f}$. It is a free $E$-module of rank 2. In [Mom81, Theorem 3.1 i)] Momose proves the following result.

Theorem 2.3.7. $\operatorname{End}\left(M_{B}\right)$ contains a sub-algebra isomorphic to $X$.

In fact Momose's paper precedes Scholl's work [Sch90] by about ten years so the motive $M_{f}$ was not available to him at the time. Momose was therefore forced to work with the realizations of $M_{f}$. However as we now show some of Momose's ideas (as well as some of Ribet's and Shimura's) can be extended to show that $X_{f}$ itself contains a crossed product algebra. We have:

Theorem 2.3.8. If $f$ has weight $k>2$ then $X_{f}$ contains a sub-algebra isomorphic to $X$.

To order to prove the theorem we state the following proposition. Let $g=$ $\sum_{(n, N)=1} a_{n} q^{n}$ be the eigenform of level $N^{2}$ introduced in the previous section and let $M_{g}$ be the associated motive. Let us set $X_{g}=\operatorname{End}\left(M_{g}\right) \otimes \mathbb{Q}$.

Proposition 2.3.9. If $N \geq 3$ then $X_{f}$ is isomorphic to $X_{g}$.

Proof of Theorem 2.3.8. During this proof let $k+2$ be the weight of $f$ and $n=N$ be the level. This change of notation is made as in the previous section to conform to the notation used by Scholl in [Sch90]. The case $k=0$ is Theorem 2.3.6 so we shall assume that $k>0$. The theorem is vacuously true in the cases $n=1$ and 2 since in these cases $X$ is the trivial algebra. We shall therefore assume that $n \geq 3$.

We shall show that $X_{g}$ contains a sub-algebra isomorphic to $X$. The proof will proceed via several lemmas. Theorem 2.3.8 will then follow from Proposition 2.3.9 which we prove after we prove the theorem.

Recall that if $\gamma \in \mathrm{GL}_{2}(\mathbb{Q})$ has positive determinant then $\gamma$ induces a slashing operation on cusp forms $h$ of weight $\nu$ given by

$$
\left.h\right|_{\gamma}(z)=\operatorname{det}(\gamma)^{\nu / 2} j(\gamma, z)^{-\nu} h(\gamma z)
$$

Let $S:=S_{k+2}\left(\Gamma_{2}(n)\right)$ and let $T \subset S$ be the subspace spanned by $g$ and its Galois conjugates. For each extra twist $\left(\gamma, \chi_{\gamma}\right)$ of $f$ define the twisting operator on $T$ by

$$
\eta_{\gamma}^{*}:=\sum_{u} \alpha_{u / r}^{*} \circ \chi_{\gamma}^{-1}(u)
$$

where $r$ is the conductor of $\chi_{\gamma}, u$ varies over a set of representatives of the integers prime to $r$ and $\alpha_{u / r}^{*}$ is the operation of slashing by the matrix $\alpha_{u / r}:=\left(\begin{array}{cc}1 & u / r \\ 0 & 1\end{array}\right)$. Note that $\chi_{\gamma}^{-1}(u)$, a priori an element of $E$, acts on $T$ not as scalar multiplication but via the map

$$
E \rightarrow \operatorname{End}(T), \quad e \mapsto\left(g^{\sigma} \mapsto e^{\sigma} g^{\sigma}\right) .
$$

A short computation shows that

$$
\eta_{\gamma}^{*}\left(g^{\sigma}\right)=G\left(\chi_{\gamma}^{-\sigma}\right) \cdot g^{\gamma \sigma}
$$


for all Galois conjugates $g^{\sigma}$ of $g$. Thus the $\eta_{\gamma}^{*}$ are endomorphisms of $T$. In [Rib80, Theorem (5.1)] it is shown that

$$
\begin{aligned}
e \circ \eta_{\gamma}^{*} & =\eta_{\gamma}^{*} \circ \gamma(e) \\
\eta_{\delta}^{*} \circ \eta_{\gamma}^{*} & =\eta_{\gamma \delta}^{*} \circ c(\gamma, \delta)
\end{aligned}
$$

as endomorphisms of $T$ where $c(\gamma, \delta)$ is the 2-cocycle defined in (2.3.4). Although only weight 2 forms were considered in [Rib80] the proof of these facts are independent of the weight of $g$. Thus there is a map from $X^{\mathrm{op}}$, the opposite algebra of $X$, to the sub-algebra of $\operatorname{End}(T)$ generated over $E$ by the $\eta_{\gamma}^{*}$. Since $X$ is 2-torsion in the Brauer group of $F$ (cf. [Rib81, page 273]) we see that $X^{\mathrm{op}}$ is isomorphic to $X$. In particular we obtain a map from $X$ to this sub-algebra of $\operatorname{End}(T)$. It is not hard to see that this map is an isomorphism (injectivity follows from the fact that $X$ has no non-trivial two-sided ideals and surjectivity is obvious).

We now show that the $\eta_{\gamma}^{*}$ are motivic, that is they come from endomorphisms $\eta_{\gamma}$ of the motive $M_{g}: \eta_{\gamma}^{*}=\mathrm{H}_{B}\left(\eta_{\gamma}\right)$ where $\mathrm{H}_{B}\left(\eta_{\gamma}\right)$ is the (scalar extension to $\mathbb{R}$ ) of the Betti realization of $\eta_{\gamma}$. It might be worth remarking that in this proof we follow the convention that maps that are adorned with $\mathrm{a}^{*}$ act on spaces of cusp forms. So * does not denote pull-back unless explicitly mentioned otherwise.

Fix an extra twist $\left(\gamma, \chi_{\gamma}\right)$ of $f$. In particular fix the conductor $r$ of $\chi_{\gamma}$. As remarked in the proof of Proposition 2.3.5 we have $r \mid n$. Let $\mathcal{H}$ denote the upper half plane. Since $\alpha_{1 / r}$ normalizes $\Gamma_{2}(n)$ the matrix $\alpha_{1 / r}$ induces a map $\alpha_{1 / r}$ : $\Gamma_{2}(n) \backslash \mathcal{H} \rightarrow \Gamma_{2}(n) \backslash \mathcal{H}$ given by $z \mapsto \alpha_{1 / r}(z)=z+1 / r$.

Lemma 2.3.10. The map

$$
z \mapsto\left(\mathbb{C} /(\mathbb{Z}+\mathbb{Z} z), \sigma: 1 / n^{2} \mapsto\left(\begin{array}{l}
1 \\
0
\end{array}\right), z / n^{2} \mapsto\left(\begin{array}{l}
0 \\
1
\end{array}\right)\right)
$$

where the triple consists of an elliptic curve over $\mathbb{C}$ together with a level $n$-structure, induces an isomorphism

$$
\theta: \Gamma_{2}(n) \backslash \mathcal{H} \stackrel{\sim}{\longrightarrow} \Gamma_{n}^{2} \backslash M_{n^{2}}(\mathbb{C}) .
$$

Proof. Let us show that $\theta$ is well defined. Assume that $w=\gamma z$ for $\gamma=\left(\begin{array}{ll}a & b \\ c & d\end{array}\right) \in$ $\Gamma_{2}(n)$. Then $w$ maps to

$$
\left(\mathbb{C} /(\mathbb{Z}+\mathbb{Z} w), 1 / n^{2} \mapsto\left(\begin{array}{l}
1 \\
0
\end{array}\right), w / n^{2} \mapsto\left(\begin{array}{l}
0 \\
1
\end{array}\right)\right)
$$

which, by multiplication by $c z+d$, is isomorphic to the tuple

$$
\left(\mathbb{C} /(\mathbb{Z}+\mathbb{Z} z), 1 / n^{2} \mapsto\left(\begin{array}{c}
a \\
-c
\end{array}\right), z / n^{2} \mapsto\left(\begin{array}{c}
-b \\
d
\end{array}\right)\right)
$$

where $a, b$, etc. also denote the images of $a, b$, etc. in $\mathbb{Z} / n^{2}$. Since $\left(\begin{array}{cc}a & -b \\ -c & d\end{array}\right) \in \Gamma_{n}^{2}$ the $\Gamma_{n}^{2}$-classes to which $w$ and $z$ map under $\theta$ are the same. This shows that $\theta$ is well defined. To show injectivity assume $\theta(z)$ and $\theta(w)$ are $\Gamma_{n}^{2}$-equivalent. Then

$$
\begin{aligned}
& \left(\mathbb{C} /(\mathbb{Z}+\mathbb{Z} z), 1 / n^{2} \mapsto\left(\begin{array}{l}
1 \\
0
\end{array}\right), z / n^{2} \mapsto\left(\begin{array}{l}
0 \\
1
\end{array}\right)\right) \\
& \quad \sim\left(\mathbb{C} /(\mathbb{Z}+\mathbb{Z} w), 1 / n^{2} \mapsto\left(\begin{array}{l}
1 \\
0
\end{array}\right), w / n^{2} \mapsto\left(\begin{array}{l}
0 \\
1
\end{array}\right)\right)
\end{aligned}
$$

where $\sim$ denotes $\Gamma_{n}^{2}$-equivalence. In particular we see that

$$
\mathbb{C} /(\mathbb{Z}+\mathbb{Z} w) \cong \mathbb{C} /(\mathbb{Z}+\mathbb{Z} z)
$$

so that $w=\gamma z$ for some $\gamma=\left(\begin{array}{ll}a & b \\ c & d\end{array}\right) \in \mathrm{SL}_{2}(\mathbb{Z})$. As above

$$
\begin{aligned}
& \left(\mathbb{C} /(\mathbb{Z}+\mathbb{Z} z), 1 / n^{2} \mapsto\left(\begin{array}{c}
1 \\
0
\end{array}\right), z / n^{2} \mapsto\left(\begin{array}{l}
0 \\
1
\end{array}\right)\right) \\
& \quad \sim\left(\mathbb{C} /(\mathbb{Z}+\mathbb{Z} w), 1 / n^{2} \mapsto\left(\begin{array}{c}
a \\
-c
\end{array}\right), w / n^{2} \mapsto\left(\begin{array}{c}
-b \\
d
\end{array}\right)\right) .
\end{aligned}
$$


This means that there is a $g \in \Gamma_{n}^{2}$ such that $g \cdot\left(\begin{array}{cc}a & -b \\ -c & d\end{array}\right)=1$. One easily sees that this forces $g$ to lie in $\Gamma_{2}(n)$ so that $w$ and $z$ define the same $\Gamma_{2}(n)$-class. This proves that $\theta$ is injective. Finally to show surjectivity let $(E, \sigma) \in M_{n^{2}}(\mathbb{C})$. Then $E \cong \mathbb{C} /(\mathbb{Z}+\mathbb{Z} z)$ for some $z \in \mathcal{H}$. Since $\Gamma_{n^{2}} \subset \Gamma_{n}^{2}$ we may always assume that the level structure induced by $\sigma$ on $\mathbb{C} /(\mathbb{Z}+\mathbb{Z} z)$ differs by the standard level structure by an element $\bar{\gamma} \in \mathrm{SL}_{2}\left(\mathbb{Z} / n^{2}\right)$. Then by replacing $z$ by $\gamma z$ where $\gamma$ is a lift of $\gamma$ to $\mathrm{SL}_{2}(\mathbb{Z})$ we see that $\theta$ takes $z$ to the $\Gamma_{n}^{2}$-class of $(E, \sigma)$. This proves that $\theta$ is surjective.

In view of the lemma it is reasonable to expect that $\alpha_{1 / r}$ has a motivic avatar. This is in fact true: it is a correspondence $\alpha: M_{n^{2}} \rightarrow M_{n^{2}}$ which we now define. Fix $(E, \sigma) \in M_{n^{2}}$. Let $d_{\sigma}$ be the integer $\bmod n^{2}$ defined by

$$
e_{n^{2}}\left(\sigma^{-1}(1,0), \sigma^{-1}(0,1)\right)^{d_{\sigma}}=\zeta_{n^{2}}
$$

where $e_{n^{2}}: E\left[n^{2}\right] \times E\left[n^{2}\right] \rightarrow \mu_{n^{2}}$ is the Weil paring and $\zeta_{n^{2}}=e^{2 \pi i / n^{2}} \in \mu_{n^{2}}$.

Assume momentarily that $d_{\sigma}=1$. Let $H$ denote the subgroup of $E\left[r^{2}\right] \subset E\left[n^{2}\right]$ defined by

$$
H=\sigma^{-1}\left\langle\left(\begin{array}{c}
n^{2} / r^{2} \\
n^{2} / r
\end{array}\right),\left(\begin{array}{c}
n^{2} / r \\
0
\end{array}\right)\right\rangle .
$$

Let $E^{\prime}=E / H$ and let $p: E \rightarrow E^{\prime}$ denote the canonical projection. We now define an $n^{2}$-level structure $\sigma^{\prime}$ on $E^{\prime}$ as follows. Choose any extension $\hat{\sigma}: E\left[r^{2} n^{2}\right] \stackrel{\sim}{\longrightarrow}$ $\left(\mathbb{Z} / r^{2} n^{2}\right)^{2}$ of $\sigma$, that is choose $\hat{\sigma}$ making the following diagram commute:

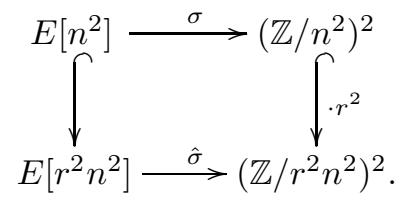

Let $\overline{\hat{\sigma}}: E\left[r^{2} n^{2}\right] / H \stackrel{\sim}{\longrightarrow}\left(\mathbb{Z} / r^{2} n^{2}\right)^{2} / \hat{\sigma}(H)$ be the map induced by $\hat{\sigma}$. It is not difficult to see that $\hat{\sigma}(H)$ is the kernel of the endomorphism of $\left(\mathbb{Z} / r^{2} n^{2}\right)^{2}$ given by $\left(\begin{array}{cc}r & -1 \\ 0 & r\end{array}\right)$. The map defined as the composition of the maps

$$
E / H\left[n^{2}\right] \hookrightarrow E\left[r^{2} n^{2}\right] / H \stackrel{\overline{\hat{\sigma}}}{\sim}\left(\mathbb{Z} / r^{2} n^{2}\right)^{2} / \hat{\sigma}(H) \stackrel{\left(\begin{array}{cc}
r & -1 \\
0 & r
\end{array}\right)}{\longrightarrow}\left(\mathbb{Z} / r^{2} n^{2}\right)^{2}
$$

actually takes values in the $n^{2}$ torsion of $\left(\mathbb{Z} / r^{2} n^{2}\right)^{2}$, that is, in the image of $\cdot r^{2}$ : $\left(\mathbb{Z} / n^{2}\right)^{2} \hookrightarrow\left(\mathbb{Z} / r^{2} n^{2}\right)^{2}$. Thus the above sequence of maps induces a map

$$
E / H\left[n^{2}\right] \rightarrow\left(\mathbb{Z} / n^{2}\right)^{2},
$$

which further is clearly an isomorphism. Denote this map by $\sigma^{\prime}$.

The above construction of $\sigma^{\prime}$ depends on a choice: the choice of the extension $\hat{\sigma}$ of $\sigma$. Let $\Sigma^{\prime}=\Sigma^{\prime}(\sigma)$ denote the set of all distinct $\sigma^{\prime}$ s obtained via the construction above. Since any two extensions $\hat{\sigma}_{1}$ and $\hat{\sigma}_{2}$ of $\sigma$ that make (2.3.11) commute differ by an element of $A \in \mathrm{GL}_{2}\left(\mathbb{Z} / r^{2} n^{2}\right)$ with $A \equiv 1 \bmod n^{2}$, a short computation shows that any two elements $\sigma_{1}^{\prime}$ and $\sigma_{2}^{\prime}$ in $\Sigma^{\prime}$ differ by an element of $\Gamma_{n}^{2}$. Let $s$ denote the cardinality of $\Sigma^{\prime}$. Then $s$ is independent of $\sigma$ and $E$, though it depends on $n$ and $r$.

Now assume that $d_{\sigma}$ is arbitrary. There is a unique element $\gamma_{\sigma} \in \Gamma_{n^{2}}$ such that $d_{\gamma_{\sigma} \sigma}=1$. In fact a short computation shows that $\gamma_{\sigma}=\left(\begin{array}{cc}d_{\sigma}^{-1} & 0 \\ 0 & 1\end{array}\right)$. Applying the above construction with $\sigma$ replaced by $\gamma_{\sigma} \sigma$ we again obtain $E^{\prime}$ and $\Sigma^{\prime}$. 
Now define

$$
\begin{aligned}
\alpha: M_{n^{2}} & \rightarrow M_{n^{2}} \\
(E, \sigma) & \mapsto \frac{1}{s} \sum_{\Sigma^{\prime}\left(\gamma_{\sigma} \sigma\right)}\left(E^{\prime},\left(\gamma_{\sigma} \sigma\right)^{\prime}\right) .
\end{aligned}
$$

The correspondence $\alpha$ is evidently defined over $\mathbb{Q}\left(\zeta_{n^{2}}\right)$. For a point $(E, \sigma) \in M_{n^{2}}$, let $[E, \sigma]$ denotes its class in $\Gamma_{n}^{2} \backslash M_{n^{2}}$. Then we have:

Lemma 2.3.14. The correspondence $\alpha: M_{n^{2}} \rightarrow M_{n^{2}}$ induces a map

$$
\alpha: \Gamma_{n}^{2} \backslash M_{n^{2}} \rightarrow \Gamma_{n}^{2} \backslash M_{n^{2}}
$$

which takes $[E, \sigma]$ to $\left[E^{\prime},\left(\gamma_{\sigma} \sigma\right)^{\prime}\right]$.

Proof. As we have remarked above any two elements of $\Sigma^{\prime}$ differ by an element of $\Gamma_{n}^{2}$. So the $s$ distinct points $\left(E^{\prime},\left(\gamma_{\sigma} \sigma\right)^{\prime}\right)$ become one point $\left[E^{\prime},\left(\gamma_{\sigma} \sigma\right)^{\prime}\right]$ in the quotient. We now show that $\alpha$ on the quotient $\Gamma_{n}^{2} \backslash M_{n^{2}}$ is well defined. To do this we must show that if $\gamma \in \Gamma_{n}^{2}$ then $[\alpha(E, \sigma)]=[\alpha(E, \gamma \sigma)]$. Since $\Gamma_{n}^{2}=\Gamma_{n^{2}} \overline{\Gamma_{2}(n)}$ where ${ }^{-}$denotes the reduction map $\mathrm{SL}_{2}(\mathbb{Z}) \rightarrow \mathrm{SL}_{2}\left(\mathbb{Z} / n^{2}\right)$ it suffices to show this in the two special cases: $\gamma \in \Gamma_{n^{2}}$ and $\gamma \in \overline{\Gamma_{2}(n)}$. So assume that $\gamma \in \Gamma_{n^{2}}$. Then $d_{\gamma \sigma}=\operatorname{det}(\gamma) d_{\sigma}$ and

$$
\gamma_{\gamma \sigma}=\gamma_{\sigma} \cdot\left(\begin{array}{cc}
\operatorname{det}(\gamma)^{-1} & 0 \\
0 & 1
\end{array}\right)=\gamma_{\sigma} \gamma^{-1}
$$

so that $\left(E, \gamma_{\gamma \sigma} \gamma \sigma\right)=\left(E, \gamma_{\sigma} \sigma\right)$. So clearly $[\alpha(E, \gamma \sigma)]=[\alpha(E, \sigma)]$. On the other hand assume that $\gamma \in \overline{\Gamma_{2}(n)}$. Since $\alpha(E, \gamma \sigma)=\alpha\left(E, \gamma^{\prime} \gamma_{\sigma} \sigma\right)$ for some $\gamma^{\prime} \in \overline{\Gamma_{2}(n)}$ and $\alpha(E, \sigma)=\alpha\left(E, \gamma_{\sigma} \sigma\right)$ by definition we may as well assume that $d_{\sigma}=1$. A short computation shows that elements of $\overline{\Gamma_{2}(n)}$ fix the subgroup

$$
\left\langle\left(\begin{array}{c}
n^{2} / r^{2} \\
n^{2} / r
\end{array}\right),\left(\begin{array}{c}
n^{2} / r \\
0
\end{array}\right)\right\rangle
$$

of $\left(\mathbb{Z} / n^{2}\right)^{2}$. In particular $E^{\prime}=E / H$ is the same whether we define $H$ as the preimage of the above subgroup under $\gamma \sigma$ or under $\sigma$. Now pick any lift $\hat{\gamma}$ of $\gamma$ to $\Gamma_{2}(n) \subset \mathrm{SL}_{2}(\mathbb{Z})$. If $\gamma^{\prime}$ is defined by

$$
\gamma^{\prime}=\left(\begin{array}{cc}
r & -1 \\
0 & r
\end{array}\right) \hat{\gamma}\left(\begin{array}{cc}
r & -1 \\
0 & r
\end{array}\right)^{-1}
$$

then $\bar{\gamma}^{\prime} \in \Gamma_{n}^{2}$ and another short computation shows that $\Sigma^{\prime}(\gamma \sigma)=\bar{\gamma}^{\prime} \circ \Sigma^{\prime}(\sigma)$. In sum we see that $[\alpha(E, \gamma \sigma)]=\left[E^{\prime},(\gamma \sigma)^{\prime}\right]=\left[E^{\prime}, \bar{\gamma}^{\prime} \cdot \sigma^{\prime}\right]=\left[\bar{\gamma}^{\prime} \cdot\left(E^{\prime}, \sigma^{\prime}\right)\right]=\left[\bar{\gamma}^{\prime} \cdot \alpha(E, \sigma)\right]=$ $[\alpha(E, \sigma)]$. This shows that the map $\alpha$ is well defined on the quotient $\Gamma_{n}^{2} \backslash M_{n^{2}}$ and proves the lemma.

Clearly the morphism $\alpha$ on the quotient $\Gamma_{n}^{2} \backslash M_{n^{2}}$ commutes with the correspondence $\alpha$ defined in (2.3.13) with respect to the projection map $M_{n^{2}} \rightarrow \Gamma_{n}^{2} \backslash M_{n^{2}}$. Moreover as the following lemma shows the map $\alpha$ on $\Gamma_{n}^{2} \backslash M_{n^{2}}$ is the moduli theoretic version of $\alpha_{1 / r}$.

Lemma 2.3.16. The following diagram commutes:

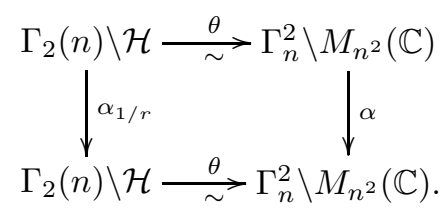


Proof. Let $z \in \mathcal{H}$. Then

$$
\begin{aligned}
\theta([z]) & =\left[\left(\mathbb{C} /(\mathbb{Z}+\mathbb{Z} z), 1 / n^{2} \mapsto\left(\begin{array}{l}
1 \\
0
\end{array}\right), z / n^{2} \mapsto\left(\begin{array}{l}
0 \\
1
\end{array}\right)\right)\right] \\
& =\left[\left(E:=\mathbb{C} /(\mathbb{Z} r+\mathbb{Z} r z), \sigma: r / n^{2} \mapsto\left(\begin{array}{l}
1 \\
0
\end{array}\right), r z / n^{2} \mapsto\left(\begin{array}{l}
0 \\
1
\end{array}\right)\right)\right]
\end{aligned}
$$

where the second equality is induced by multiplication by $r$. Clearly $d_{\sigma}=1$. A short computation shows that $H$ is the subgroup of $E\left[r^{2}\right]$ generated by 1 and $z+1 / r$. To define $\alpha$ we must write down $\left(E^{\prime}, \sigma^{\prime}\right)$ where $E^{\prime}=E / H$ and $\sigma^{\prime}$ is the level $n^{2}$ structure on $E^{\prime}$ defined in (2.3.12). To this end let $\hat{\sigma}$ be the $r^{2} n^{2}$-level structure on $E$ defined by

$$
\hat{\sigma}: 1 / r n^{2} \mapsto\left(\begin{array}{l}
1 \\
0
\end{array}\right), z / r n^{2} \mapsto\left(\begin{array}{l}
0 \\
1
\end{array}\right)
$$

Then $E / H\left[n^{2}\right]=\hat{\sigma}^{-1}\left\langle\left(\begin{array}{l}r \\ 0\end{array}\right),\left(\begin{array}{l}1 \\ r\end{array}\right)\right\rangle / H$ and a short computation shows that

$$
\begin{aligned}
\sigma^{\prime}\left(\hat{\sigma}^{-1}\left(\begin{array}{l}
r \\
0
\end{array}\right)+H\right) & =\left(\begin{array}{l}
1 \\
0
\end{array}\right) \in\left(\mathbb{Z} / n^{2}\right), \\
\sigma^{\prime}\left(\hat{\sigma}^{-1}\left(\begin{array}{l}
1 \\
r
\end{array}\right)+H\right) & =\left(\begin{array}{l}
0 \\
1
\end{array}\right) \in\left(\mathbb{Z} / n^{2}\right) .
\end{aligned}
$$

Now $\alpha\left([\theta([z]))=\alpha([(E, \sigma)])=\left[\left(E^{\prime}, \sigma^{\prime}\right)\right]\right.$ where $E^{\prime}$ and $\sigma^{\prime}$ are defined as above. On the other hand

$$
\begin{aligned}
\theta\left(\left[\alpha_{1 / r}(z)\right]\right) & =\left[\left(\mathbb{C} /(\mathbb{Z}+\mathbb{Z}(z+1 / r)), 1 / n^{2} \mapsto\left(\begin{array}{l}
1 \\
0
\end{array}\right),(z+1 / r) / n^{2} \mapsto\left(\begin{array}{l}
0 \\
1
\end{array}\right)\right)\right] \\
& =\left[\left(E^{\prime}, \sigma^{\prime}\right)\right]
\end{aligned}
$$

since $1 / n^{2}=\hat{\sigma}^{-1}\left(\begin{array}{c}r \\ 0\end{array}\right)$ and $(z+1 / r) / n^{2}=\hat{\sigma}^{-1}\left(\begin{array}{l}1 \\ r\end{array}\right)$.

We now extend the definition of $\alpha$ to the cusps. To do this we need to recall some facts from [DR73]. Recall that a Néron $n$-gon is a polygon $C$ obtained by gluing $n$ marked copies of $\mathbb{P}^{1}$ together, the point 0 of the $i$-th copy being glued to the point $\infty$ of the $i+1$-th copy. Thus $C$ is a curve of genus 1 whose normalization is $\mathbb{P}^{1} \times \mathbb{Z} / n$. The inverse image of $C^{\text {reg }}$ in its normalization is $\mathbb{G}_{m} \times \mathbb{Z} / n$. This has a natural group structure whose $n$-torsion subgroup, which we denote by $C[n]$, is $\mu_{n} \times \mathbb{Z} / n$. A level $n$-structure on $C$ is an isomorphism $\sigma: C[n] \stackrel{\sim}{\rightarrow}(\mathbb{Z} / n)^{2}$.

Now consider the following two types of automorphisms of $\mathbb{P}^{1} \times \mathbb{Z} / n$ :

$$
\tau:(x, i) \mapsto\left(x^{-1},-i\right) \text { and } \sigma_{\zeta}:(x, i) \mapsto\left(\zeta^{i} \cdot x, i\right), \text { where } \zeta \in \mu_{n} .
$$

Each of these maps descends to an automorphism of $C$, and induces an automorphism of the group $\mathbb{G}_{m} \times \mathbb{Z} / n$. The group of automorphisms $A$ generated by the above maps is in fact the full group of automorphisms of $C$ compatible with the group structure. Thus $A$ is the semi-direct product of $\{\mathrm{id}, \tau\}$ and $\mu_{n}$.

A pair $(C, \sigma)$ consisting of an $n$-gon and level $n$-structure is said to be isomorphic to another pair $\left(C, \sigma^{\prime}\right)$ if there is an element $g \in A$ such that $\sigma^{\prime}=\sigma \circ g$. It is shown in [DR73] that the curve $\bar{M}_{n}$ parameterizes isomorphism classes of generalized elliptic curves with level $n$-structure: that is, while the points of $M_{n}$ parameterize usual isomorphism classes $(E, \sigma)$ of elliptic curves with level $n$-structure, the finite set of points of $\bar{M}_{n} \backslash M_{n}$ parameterize isomorphism classes of Néron $n$-gons (which for clarity we have denoted by a different letter here, namely $C$ ) with level $n$-structure.

Recall there is a natural action of $\mathrm{GL}_{2}(\mathbb{Z} / n)$ on $\bar{M}_{n} \backslash M_{n}$ (cf. (2.2.1)). In particular $\Gamma_{n}^{2} \subset \mathrm{GL}_{2}\left(\mathbb{Z} / n^{2}\right)$ acts on $\bar{M}_{n^{2}} \backslash M_{n^{2}}$. We let $\Gamma_{n}^{2} \backslash\left(\bar{M}_{n^{2}} \backslash M_{n^{2}}\right)$ denote the quotient. Finally note that a level $n^{2}$-structure $\sigma: C\left[n^{2}\right] \rightarrow\left(\mathbb{Z} / n^{2}\right)^{2}$ is completely specified by giving its values on $\zeta_{n^{2}}:=e^{2 \pi i / n^{2}} \in \mu_{n^{2}}$ and the generator 1 of $\mathbb{Z} / n^{2}$. We can now state the following lemma. 
Lemma 2.3.17. The assignment

$$
\left(\begin{array}{l}
a \\
c
\end{array}\right) \mapsto\left(C, \sigma: \zeta_{n^{2}} \mapsto\left(\begin{array}{c}
\bar{a} \\
\bar{c}
\end{array}\right), 1 \mapsto\left(\begin{array}{c}
\bar{b} \\
\bar{d}
\end{array}\right)\right)
$$

where $a$ and $c$ are relatively prime integers, and $\left(\begin{array}{c}\bar{b} \\ \bar{d}\end{array}\right)$ is any pair such that $\left(\begin{array}{c}\bar{a} \\ \bar{c} \\ \bar{b}\end{array}\right) \in$ $\mathrm{SL}_{2}\left(\mathbb{Z} / n^{2}\right)$, induces a bijection

$$
\theta: \Gamma_{2}(n) \backslash \mathbb{P}^{1}(\mathbb{Q}) \stackrel{\sim}{\longrightarrow} \Gamma_{n}^{2} \backslash\left(\bar{M}_{n^{2}} \backslash M_{n^{2}}\right) .
$$

Proof. We first show that there is a natural bijection

$$
\Gamma(n) \backslash \mathbb{P}^{1}(\mathbb{Q}) \stackrel{\sim}{\longrightarrow} \Gamma_{n} \backslash\left(\bar{M}_{n} \backslash M_{n}\right) .
$$

Let $V_{n}$ be the set of elements of $(\mathbb{Z} / n)^{2}$ of exact order $n$. Then the map induced by $\left(\begin{array}{l}a \\ c\end{array}\right) \mapsto\left(\begin{array}{c}\bar{a} \\ \bar{c}\end{array}\right) \in V_{n}$, where $a$ and $c$ are relatively prime integers, induces a bijection

$$
\Gamma(n) \backslash \mathbb{P}^{1}(\mathbb{Q}) \stackrel{\sim}{\longrightarrow} V_{n} / \pm 1 .
$$

On the other hand the map induced by taking $\left(\frac{\bar{a}}{\bar{c}}\right) \in V_{n}$ to the pair $(C, \sigma)$, where $\sigma$ is the level $n$-structure defined by $\zeta_{n}=e^{2 \pi i / n} \mapsto\left(\frac{\bar{a}}{\bar{c}}\right), 1 \mapsto\left(\begin{array}{c}\bar{b} \\ \bar{d}\end{array}\right)$ with $\left(\frac{\bar{b}}{\bar{d}}\right)$ any

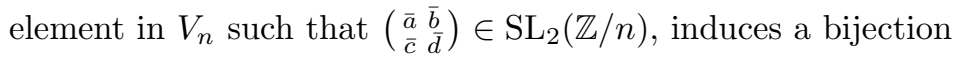

$$
V_{n} / \pm 1 \stackrel{\sim}{\longrightarrow} \Gamma_{n} \backslash\left(\bar{M}_{n} \backslash M_{n}\right) \text {. }
$$

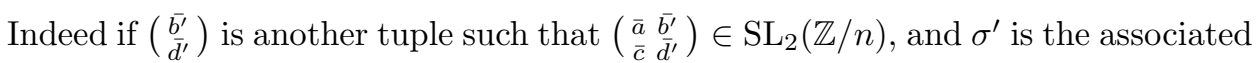
level structure, then taking $l=d b^{\prime}-b d^{\prime}$, a short computation shows that $\sigma^{\prime}=\sigma \circ \sigma_{\zeta_{n}^{l}}$, so the pair $\left(C, \sigma^{\prime}\right)$ is isomorphic to the pair $(C, \sigma)$. Similarly, since $\sigma \circ \tau=-\sigma$, we see that $(C,-\sigma)$ is isomorphic to $(C, \sigma)$ and so the map does indeed factor through the quotient $V_{n} / \pm 1$. Thus (2.3.19) is well defined. To show it is injective suppose that $\left(\begin{array}{c}\bar{a} \\ \bar{c}\end{array}\right)$ and $\left(\frac{\overline{a^{\prime}}}{c^{\prime}}\right)$ are two elements of $V_{n}$, whose associated level structures have matrices

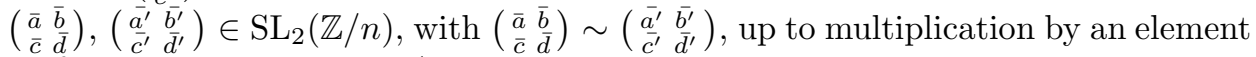
$\gamma$ of $\Gamma_{n}$, where $\sim$ denotes $A$-equivalence. Then, comparing determinants, we must have $\gamma=1$. Moreover, since the effect of elements of $A$ on the first column of the matrices above is at most alteration by a sign we see that $\left(\frac{\bar{a}}{\bar{c}}\right)$ and $\left(\frac{\overline{a^{\prime}}}{c^{\prime}}\right)$ define the same class in $V_{n} / \pm 1$. Finally the surjectivity of (2.3.19) is clear, since an arbitrary level $n$-structure $\sigma$ is always, up to a unique element of $\Gamma_{n}$, of 'determinant equal to 1 '. This proves that (2.3.19) is a bijection, and therefore that (2.3.18) is a bijection. Replacing $n$ with $n^{2}$ in (2.3.18) and taking $\Gamma_{2}(n)$-invariants we get the bijection $\theta$ of the lemma.

We are now ready to define the action of $\alpha$ on the cusps. Since $\alpha_{1 / r}$ normalizes $\Gamma_{2}(n)$, it induces a map $\alpha_{1 / r}: \Gamma_{2}(n) \backslash \mathbb{P}^{1}(\mathbb{Q}) \rightarrow \Gamma_{2}(n) \backslash \mathbb{P}^{1}(\mathbb{Q})$. We now simply define $\alpha: \Gamma_{n}^{2} \backslash\left(\bar{M}_{n^{2}} \backslash M_{n^{2}}\right) \rightarrow \Gamma_{n}^{2} \backslash\left(\bar{M}_{n^{2}} \backslash M_{n^{2}}\right)$ as the avatar of $\alpha_{1 / r}$ with respect to the bijection $\theta$ of the lemma above. More precisely, we define $\alpha$ so that the following diagram commutes:

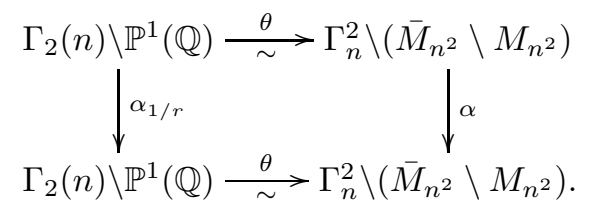

We also define $\alpha$ as a correspondence on $\bar{M}_{n^{2}} \backslash M_{n^{2}}$ so that it lifts the map $\alpha$ on the quotient $\Gamma_{n}^{2} \backslash\left(\bar{M}_{n^{2}} \backslash M_{n^{2}}\right)$. More precisely, if [ ] denotes $\Gamma_{n}^{2}$-class and 
$\alpha([C, \sigma])=\left[C, \sigma^{\prime}\right]$ then define

$$
\begin{aligned}
\alpha: \bar{M}_{n^{2}} \backslash M_{n^{2}} & \rightarrow \bar{M}_{n^{2}} \backslash M_{n^{2}} \\
(C, \sigma) & \mapsto \frac{1}{s^{\prime}} \sum_{\gamma \in \Gamma_{n}^{2}}\left(C, \gamma \circ \sigma^{\prime}\right)
\end{aligned}
$$

where $s^{\prime}$ is the cardinality of $\Gamma_{n}^{2}$.

Set $\overline{\mathcal{H}}:=\mathcal{H} \cup \mathbb{P}^{1}(\mathbb{Q})$. In view of (2.3.13) and (2.3.20) we have now defined a correspondence $\alpha: \bar{M}_{n^{2}} \rightarrow \bar{M}_{n^{2}}$ which, by lemma 2.3.16 and the commutative square above, makes the following diagram commute:

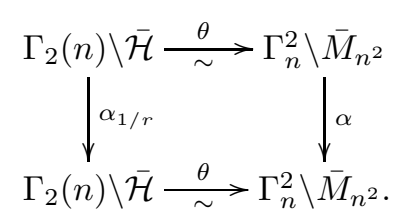

We now extend the definition of $\alpha$ to a correspondence $X_{n^{2}} \rightarrow X_{n^{2}}$ as follows:

$$
\alpha:(e \in E, \sigma) \mapsto \frac{1}{s r^{2} n^{2}} \sum_{t \in E\left[r^{2} n^{2}\right]} \sum_{\Sigma^{\prime}\left(\gamma_{\sigma} \sigma\right)}\left(p(e)+p(t) \in E^{\prime},\left(\gamma_{\sigma} \sigma\right)^{\prime}\right)
$$

where the sum on $t$ is over all $r^{2} n^{2}$ torsion points, and we recall that $p: E \rightarrow E^{\prime}$ is the canonical projection. We further extend $\alpha$ to a correspondence $\bar{X}_{n^{2}} \rightarrow \bar{X}_{n^{2}}$, by defining $\alpha$ on the fibers lying over the cusps as follows:

$$
\alpha:(c \in C, \sigma) \mapsto \begin{cases}(c \in C, \sigma) & \text { if } c \notin C^{\mathrm{reg}}, \\ \frac{1}{s^{\prime} n^{2}} \sum_{t \in C\left[n^{2}\right]} \sum_{\gamma \in \Gamma_{n}^{2}}\left(c+t \in C, \gamma \circ \sigma^{\prime}\right) & \text { if } c \in C^{\mathrm{reg}} .\end{cases}
$$

The self-correspondence $\alpha$ of $\bar{X}_{n^{2}}$ extends in a natural way to a self-correspondence of $\bar{X}_{n^{2}}^{k}$ which we continue to denote by $\alpha$.

Lemma 2.3.21. $\alpha: \bar{X}_{n^{2}}^{k} \rightarrow \bar{X}_{n^{2}}^{k}$ commutes with $\Pi_{\varepsilon}$.

Proof. The correspondence $\alpha$ is easily seen to commute with the actions of $\mu_{2}^{k}$ and $\Sigma_{k}$. Therefore to prove the lemma it suffices to show that

$$
\alpha \circ \sum_{g \in\left(\mathbb{Z} / n^{2}\right)^{2}} g=\left(\sum_{g \in\left(\mathbb{Z} / n^{2}\right)^{2}} g\right) \circ \alpha .
$$


Fix a point $(e \in E, \sigma) \in X_{n^{2}}$. Then:

$$
\begin{aligned}
& \alpha\left(\sum_{g \in\left(\mathbb{Z} / n^{2}\right)^{2}} g(e, \sigma)\right) \\
& =\alpha\left(\sum_{g \in\left(\mathbb{Z} / n^{2}\right)^{2}}\left(e+\sigma^{-1}(g), \sigma\right)\right) \\
& =\frac{1}{s r^{2} n^{2}} \sum_{t \in E\left[r^{2} n^{2}\right]} \sum_{\Sigma^{\prime}\left(\gamma_{\sigma} \sigma\right)} \sum_{g \in\left(\mathbb{Z} / n^{2}\right)^{2}}\left(p(e)+p\left(\sigma^{-1}(g)\right)+p(t),\left(\gamma_{\sigma} \sigma\right)^{\prime}\right) \\
& =\frac{n^{4}}{s r^{2} n^{2}} \sum_{t \in E\left[r^{2} n^{2}\right]} \sum_{\Sigma^{\prime}\left(\gamma_{\sigma} \sigma\right)}\left(p(e)+p(t),\left(\gamma_{\sigma} \sigma\right)^{\prime}\right) \\
& =\frac{1}{s r^{2} n^{2}} \sum_{t \in E\left[r^{2} n^{2}\right]} \sum_{\Sigma^{\prime}\left(\gamma_{\sigma} \sigma\right)} \sum_{g \in\left(\mathbb{Z} / n^{2}\right)^{2}}\left(p(e)+p(t)+\left(\gamma_{\sigma} \sigma\right)^{\prime-1}(g),\left(\gamma_{\sigma} \sigma\right)^{\prime}\right) \\
& =\left(\sum_{g \in\left(\mathbb{Z} / n^{2}\right)^{2}} g\right) \circ \alpha(e, \sigma),
\end{aligned}
$$

where the third (resp. fourth) equality follows from the fact that $p\left(\sigma^{-1}(g)\right) \in$ $p\left(E\left[r^{2} n^{2}\right]\right)$ (resp. $\left.\left(\gamma_{\sigma} \sigma\right)^{\prime-1}(g) \in p\left(E\left[r^{2} n^{2}\right]\right)\right)$. The proof for a point $(c \in C, \sigma)$ where $C$ is a Néron $n^{2}$-gon is similar and is omitted. This proves the lemma.

By the canonical nature of the desingularization of $\bar{X}_{n^{2}}^{k}$ the correspondence $\alpha$ extends to a correspondence

$$
\alpha: \overline{\bar{X}}_{n^{2}}^{k} \rightarrow \overline{\bar{X}}_{n^{2}}^{k}
$$

By the lemma above $\alpha$ induces a morphism of the motive $W_{n^{2}}=\left(\overline{\bar{X}}_{n^{2}}^{k}, \Pi_{\varepsilon}\right)$ which we call $\alpha$ again.

Given a vector space $V$ over $\mathbb{Q}$ with an action of the group $\Gamma_{k}$ let $V(\epsilon)$ denote the image of $V$ under the endomorphism of $V$ induced by the projector $\Pi_{\varepsilon}$. Let $\mathrm{H}_{B}\left(W_{n^{2}}\right):=\mathrm{H}^{*}\left(\bar{X}_{n^{2}}^{k}(\mathbb{C}), \mathbb{Q}\right)(\varepsilon)$ be the Betti realization of the motive $W_{n^{2}}$. Scholl has proved (cf. [Sch90, Theorem 1.2.1])

$$
\text { Sch : } \mathrm{H}_{B}\left(W_{n^{2}}\right) \stackrel{\sim}{\longrightarrow} \mathrm{H}^{1}\left(\bar{M}_{n^{2}}(\mathbb{C}), j_{*} \operatorname{Sym}^{k} R^{1} \pi_{*} \mathbb{Q}\right) .
$$

Let $\mathrm{H}_{B}(\alpha): \mathrm{H}_{B}\left(W_{n^{2}}\right) \rightarrow \mathrm{H}_{B}\left(W_{n^{2}}\right)$ denote the Betti realization of $\alpha$.

Lemma 2.3.22. The correspondence $\alpha: \bar{X}_{n^{2}}^{k} \rightarrow \bar{X}_{n^{2}}^{k}$ quasi-commutes with elements of $\Gamma_{n}^{2}$, that is, given $\gamma \in \Gamma_{n}^{2}$, there exists a $\gamma^{\prime} \in \Gamma_{n}^{2}$ making the following diagram commute:

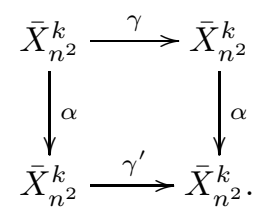

Proof. It suffices to prove the lemma when $k=1$ since the general case is proved similarly. Now we have already shown in the course of proving Lemma 2.3.14 that $\alpha: M_{n^{2}} \rightarrow M_{n^{2}}$ quasi-commutes with elements of $\Gamma_{n}^{2}$. It is a simple check to see 
that this implies that $\alpha: \bar{X}_{n^{2}} \rightarrow \bar{X}_{n^{2}}$ quasi-commutes with elements of $\Gamma_{n}^{2}$. The lemma follows.

By the lemma $\alpha$ induces an endomorphism of $W_{n^{2}}^{\Gamma_{n}^{2}}$. Let us continue to write $\mathrm{H}_{B}(\alpha)$ for the endomorphism of $\mathrm{H}_{B}\left(W_{n^{2}}^{\Gamma_{n}^{2}}\right)=\mathrm{H}_{B}\left(W_{n^{2}}\right)^{\Gamma_{n}^{2}}$ induced by $\alpha$. Moreover the lemma shows that the isomorphism of Scholl above induces the following isomorphism:

$$
\mathrm{H}_{B}\left(W_{n^{2}}^{\Gamma_{n}^{2}}\right) \stackrel{\mathrm{Sch}}{\sim} \mathrm{H}^{1}\left(\bar{M}_{n^{2}}, j_{*} \operatorname{Sym}^{k} R^{1} \pi_{*} \mathbb{Q}\right)^{\Gamma_{n}^{2}}
$$

which we again denote by Sch.

Let $L(k, \mathbb{R})$ denote the space of homogeneous polynomials of degree $k$ in the two variables $X$ and $Y$ with coefficients in $\mathbb{R}$. Then $L(k, \mathbb{R})$ is a $\Gamma_{2}(n)$-module via

$$
\gamma \cdot P\left(\begin{array}{l}
x \\
y
\end{array}\right)=P\left(\gamma^{-1}\left(\begin{array}{l}
x \\
y
\end{array}\right)\right)
$$

for $\gamma \in \Gamma_{2}(n)$ and $P\left(\begin{array}{l}x \\ y\end{array}\right) \in L(k, \mathbb{R})$. Let $\mathrm{H}_{\mathrm{p}}^{1}\left(\Gamma_{2}(n), L(k, \mathbb{R})\right)$ denote the corresponding parabolic cohomology group. Recall that there is an isomorphism which we call the Shimura isomorphism and which we denote by Sh (cf. [Del69]):

$$
\begin{aligned}
\text { Sh }: S & \stackrel{\sim}{\longrightarrow} \mathrm{H}_{\mathrm{p}}^{1}\left(\Gamma_{2}(n), L(k, \mathbb{R})\right) \\
h & \mapsto\left[c_{h}: \gamma \mapsto \int_{z_{0}}^{\gamma z_{0}} \omega(h)\right]
\end{aligned}
$$

where $\omega(h)=\operatorname{Re}\left(2 \pi i h(z)(X-z Y)^{k} d z\right)$ and $z_{0}$ is a base point in $\mathcal{H}$. Changing the base point changes $c_{h}$ by a coboundary and Sh is well defined.

Give $L(k, \mathbb{R})$ the discrete topology and let $\tilde{L}(k, \mathbb{R})$ denote the sheaf on $\Gamma_{2}(n) \backslash \mathcal{H}$ consisting of locally constant sections of the cover

$$
\Gamma_{2}(n) \backslash(\mathcal{H} \times L(k, \mathbb{R})) \rightarrow \Gamma_{2}(n) \backslash \mathcal{H}
$$

Then there is a natural isomorphism

$$
\mathrm{H}^{1}\left(\Gamma_{2}(n), L(k, \mathbb{R})\right) \stackrel{\sim}{\longrightarrow} \mathrm{H}^{1}\left(\Gamma_{2}(n) \backslash \mathcal{H}, \tilde{L}(k, \mathbb{R})\right) .
$$

Recall that the isomorphism $\theta$ of Lemma 2.3.10 identifies $\Gamma_{2}(n) \backslash \mathcal{H}$ with $\Gamma_{n}^{2} \backslash M_{n^{2}}$. Let $\pi_{1}: \mathcal{H} \rightarrow \Gamma_{2}(n) \backslash \mathcal{H}$ and $\pi_{2}: M_{n^{2}} \rightarrow \Gamma_{n}^{2} \backslash M_{n^{2}}$ be the projection maps. We now show that $\theta$ may be used to identify the sheaf $\mathcal{F}=\tilde{L}(k, \mathbb{R})$ on $\Gamma_{2}(n) \backslash \mathcal{H}$ with the sheaf $\mathcal{G}=\operatorname{Sym}^{k} R^{1} \pi_{*} \mathbb{R}$ on $\Gamma_{n}^{2} \backslash M_{n^{2}}$. (More precisely $\mathcal{G}$ is the sub-sheaf of $\Gamma_{n^{-}}^{2}$ invariant sections of $\left.\pi_{2 *} \operatorname{Sym}^{k} R^{1} \pi_{*} \mathbb{R}\right)$. If $U$ is an open sub-set of $\Gamma_{2}(n) \backslash \mathcal{H}$ then

$$
\begin{aligned}
\mathcal{F}(U)=\{f: & \pi_{1}^{-1}(U) \rightarrow L(k, \mathbb{R}) \mid f \text { is locally constant and } \\
& \left.\quad f(\gamma z)=\gamma \cdot f(z), \text { for all } z \in \pi_{1}^{-1}(U) \text { and } \gamma \in \Gamma_{2}(n)\right\} .
\end{aligned}
$$

Here and in the discussion below $f$ is a section of $\mathcal{F}$ and should not be confused with the modular form $f$. Similarly if $V$ is an open sub-set of $\Gamma_{n}^{2} \backslash M_{n^{2}}$ then

$$
\begin{aligned}
\mathcal{G}(V)=\{g: & \pi_{2}^{-1}(V) \rightarrow \coprod_{(E, \sigma)} \operatorname{Sym}^{k} \mathrm{H}^{1}(E, \mathbb{R}) \mid g \text { is locally constant, } \\
& g(E, \sigma) \in \operatorname{Sym}^{k} \mathrm{H}^{1}(E, \mathbb{R}), \text { and } g(E, \gamma \sigma)=g(E, \sigma) \\
& \text { for all } \left.(E, \sigma) \in \pi_{2}^{-1}(V) \text { and } \gamma \in \Gamma_{n}^{2}\right\}
\end{aligned}
$$

Again $g$ is a section of $\mathcal{G}$ and should not be confused with the modular form $g$. Also $\mathrm{H}^{1}(E, \mathbb{R})$ is to be identified with $\mathrm{H}^{1}\left(E^{\prime}, \mathbb{R}\right)$ if $(E, \sigma)$ is isomorphic to $\left(E^{\prime}, \sigma^{\prime}\right)$. 
Now suppose that the open sets $U$ and $V$ correspond under the isomorphism $\theta$ of Lemma 2.3.10. For $z \in \mathcal{H}$, let

$$
\left(E_{z}, \sigma_{z}\right):=\left(\mathbb{C} /(\mathbb{Z}+\mathbb{Z} z), \sigma: 1 / n^{2} \mapsto\left(\begin{array}{l}
1 \\
0
\end{array}\right), z / n^{2} \mapsto\left(\begin{array}{l}
0 \\
1
\end{array}\right)\right)
$$

denote the elliptic curve and level structure defined in Lemma 2.3.10. Consider the dictionary

$$
\begin{aligned}
f(z) & :=g\left(E_{z}, \sigma_{z}\right), \\
g(E, \sigma) & :=f(z) \text { if }(E, \sigma) \sim\left(E_{z}, \gamma \sigma_{z}\right), \text { for } z \in \mathcal{H} \text { and } \gamma \in \Gamma_{n}^{2},
\end{aligned}
$$

between the sections $f \in \mathcal{F}(U)$ and $g \in \mathcal{G}(V)$, where we have identified the ranges of $f$ and $g$ using the explicit isomorphism

$$
\operatorname{Sym}^{k} \mathrm{H}^{1}\left(E_{z}, \mathbb{R}\right) \stackrel{\sim}{\longrightarrow} L(k, \mathbb{R})
$$

defined as follows. Identify $\mathrm{H}^{1}\left(E_{z}, \mathbb{R}\right)=\operatorname{Hom}(\mathbb{Z} \oplus \mathbb{Z} z, \mathbb{R})$ with $L(1, \mathbb{R})$ via the isomorphism $h_{1} \mapsto X, h_{z} \mapsto-Y$ where $h_{1}, h_{z} \in \operatorname{Hom}(\mathbb{Z} \oplus \mathbb{Z} z, \mathbb{R})$ is the basis dual to $1, z$. Then we take $(2.3 .27)$ to be the induced isomorphism obtained by applying $\operatorname{Sym}^{k}$, noting that $\operatorname{Sym}^{k} L(1, \mathbb{R})=L(k, \mathbb{R})$ as a $\Gamma_{2}(n)$-module.

The dictionary (2.3.26) may be used to identify the sheaves $\mathcal{F}$ and $\mathcal{G}$. We first check that it is well-defined. Let $\gamma=\left(\begin{array}{ll}a & b \\ c & d\end{array}\right) \in \Gamma_{2}(n)$. Then multiplication by $c z+d$ induces an isomorphism $E_{\gamma z} \stackrel{\sim}{\longrightarrow} E_{z}$, so that one has an induced map $\operatorname{Sym}^{k} \mathrm{H}^{1}\left(E_{z}, \mathbb{R}\right) \stackrel{\sim}{\longrightarrow} \operatorname{Sym}^{k} \mathrm{H}^{1}\left(E_{\gamma z}, \mathbb{R}\right)$ which we shall also denote by $\gamma$. Now a short computation shows that the diagram

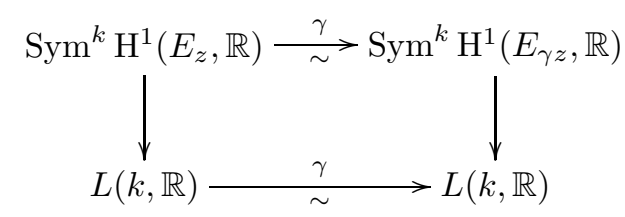

commutes, where the vertical maps are the isomorphism (2.3.27). If $\gamma \in \Gamma_{2}(n)$, then $f(\gamma z)=g\left(E_{\gamma z}, \sigma_{\gamma z}\right)=\gamma \cdot f(z)$, using the facts: $\left(E_{\gamma z}, \sigma_{\gamma z}\right) \sim\left(E_{z}, \sigma_{z}\right)$ up to multiplication by an element of $\Gamma_{n}^{2}$; (2.3.28) above commutes; and $g$ is $\Gamma_{n^{-}}^{2}$ equivariant. Thus $f$ is well defined. As for $g$, a similar check using the relation $f(\gamma z)=\gamma \cdot f(z)$, for $\gamma \in \Gamma_{2}(n)$, and (2.3.28), shows that the definition of $g$ is independent of the $\Gamma_{2}(n)$-orbit of $z$. So $g$ is also well defined. Note also that $g(E, \gamma \sigma)=g(E, \sigma)$ for $\gamma \in \Gamma_{n}^{2}$. Finally since the dictionary $f \leftrightarrow g$ is clearly a bijection, one obtains the desired identification of the sheafs $\mathcal{F}$ and $\mathcal{G}$.

It follows that there is an induced isomorphism on the level of cohomology:

$$
\mathrm{H}^{1}\left(\Gamma_{2}(n) \backslash \mathcal{H}, \tilde{L}(k, \mathbb{R})\right) \stackrel{\sim}{\longrightarrow} \mathrm{H}^{1}\left(M_{n^{2}}(\mathbb{C}), \operatorname{Sym}^{k} R^{1} \pi_{*} \mathbb{R}\right)^{\Gamma_{n}^{2}}
$$

where we have identified $\mathrm{H}^{1}\left(\Gamma_{n}^{2} \backslash M_{n^{2}}, \mathcal{G}\right)$ with $\mathrm{H}^{1}\left(M_{n^{2}}, \operatorname{Sym}^{k} R^{1} \pi_{*} \mathbb{R}\right)^{\Gamma_{n}^{2}}$. Combining this map with the map (2.3.25) we obtain an isomorphism

$$
\left.\mathrm{H}^{1}\left(\Gamma_{2}(n), L(k, \mathbb{R})\right) \stackrel{\sim}{\longrightarrow} \mathrm{H}^{1}\left(M_{n^{2}}(\mathbb{C}), \operatorname{Sym}^{k} R^{1} \pi_{*} \mathbb{R}\right)\right)^{\Gamma_{n}^{2}} .
$$

Since this isomorphism preserves the parabolic parts on both sides, namely

$$
\begin{aligned}
\mathrm{H}_{\mathrm{p}}^{1}\left(\Gamma_{2}(n), L(k, \mathbb{R})\right) & \subset \mathrm{H}^{1}\left(\Gamma_{2}(n), L(k, \mathbb{R})\right), \\
\mathrm{H}^{1}\left(\bar{M}_{n^{2}}(\mathbb{C}), j_{*} \operatorname{Sym}^{k} R^{1} \pi_{*} \mathbb{R}\right)^{\Gamma_{n}^{2}} & \subset \mathrm{H}^{1}\left(M_{n^{2}}, \operatorname{Sym}^{k} R^{1} \pi_{*} \mathbb{R}\right)^{\Gamma_{n}^{2}},
\end{aligned}
$$


we finally get an isomorphism

$$
\left.\mathrm{H}_{\mathrm{p}}^{1}\left(\Gamma_{2}(n), L(k, \mathbb{R})\right) \stackrel{\theta}{\sim} \underset{\sim}{\sim} \mathrm{H}^{1}\left(\bar{M}_{n^{2}}(\mathbb{C}), j_{*} \operatorname{Sym}^{k} R^{1} \pi_{*} \mathbb{R}\right)\right)^{\Gamma_{n}^{2}}
$$

which we shall also denote by $\theta$.

We now define $I$ to be the isomorphism which is the composition of the isomorphisms Sh, $\theta$ and the inverse of (the scalar extension to $\mathbb{R}$ of) Sch (cf. (2.3.24), (2.3.31) and (2.3.23) above):

$$
I=\mathrm{Sch}^{-1} \circ \theta \circ \mathrm{Sh}: S \stackrel{\sim}{\longrightarrow} \mathrm{H}_{B}\left(W_{n^{2}}^{\Gamma_{n}^{2}}\right) \otimes \mathbb{R} .
$$

Recall that $\mathrm{H}_{B}(\alpha)$ is the action induced by $\alpha$ on $\mathrm{H}_{B}\left(W_{n^{2}}^{\Gamma_{n}^{2}}\right)$. On the other hand the action of $\alpha_{1 / r}$ induces an action on $S$ given by slashing by $\alpha_{1 / r}$. Denote this action by $\alpha_{1 / r}^{*}$. As the following proposition shows these two actions are related via the isomorphism $I$.

Lemma 2.3.32. The following diagram commutes

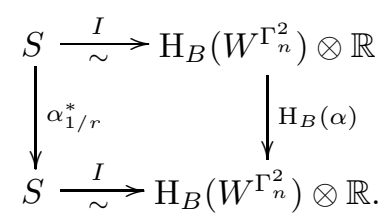

Proof. The proof follows from the commutativity of three squares, each corresponding to one of the three isomorphisms out of which $I$ is built. Each square will show that the isomorphism under consideration identifies the natural action induced by $\alpha$ on the domain and range of this isomorphism.

We start with the Shimura isomorphism. The matrix $\alpha_{1 / r}$ acts in the usual way on the parabolic cohomology group: for a parabolic 1-cocycle $c: \Gamma_{2}(n) \rightarrow L(k, \mathbb{R})$ one has

$$
\left(\alpha_{1 / r} \cdot c\right)(\gamma)=\alpha_{1 / r}^{-1} \cdot c\left(\alpha_{1 / r} \gamma \alpha_{1 / r}^{-1}\right)
$$

A short computation shows that $\alpha_{1 / r}^{-1} \cdot\left(\alpha_{1 / r}^{*} \omega(h)\right)=\omega\left(\left.h\right|_{\alpha_{1 / r}}\right)$ (where $\alpha_{1 / r}^{*}$ temporarily denotes pull-back and not slashing!) so that $\left(\alpha_{1 / r} \cdot c_{h}\right)(\gamma)=$

$$
\alpha_{1 / r}^{-1} \cdot \int_{z_{0}}^{\alpha_{1 / r} \gamma \alpha_{1 / r}^{-1} z_{0}} \omega(h)=\alpha_{1 / r}^{-1} \cdot \int_{\alpha_{1 / r}^{-1} z_{0}}^{\gamma \alpha_{1 / r}^{-1} z_{0}} \alpha_{1 / r}^{*} \omega(h)=\int_{\alpha_{1 / r}^{-1} z_{0}}^{\gamma \alpha_{1 / r}^{-1} z_{0}} \omega\left(\left.h\right|_{\alpha_{1 / r}}\right) .
$$

Since the last term is up to a coboundary just $c_{h_{\left.\right|_{1 / r}}}(\gamma)$ we see that the Shimura isomorphism identifies slashing by $\alpha_{1 / r}$ on $S$, which we have called $\alpha_{1 / r}^{*}$ (this is not pull-back!) with the action of $\alpha_{1 / r}$ on the above parabolic cohomology group. In other words the following diagram commutes:

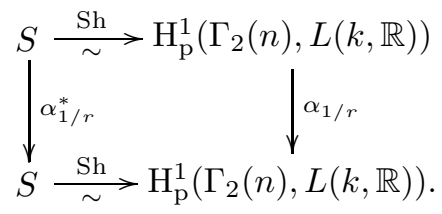

Let us now show that the isomorphism $\theta$ in (2.3.31) identifies the action of $\alpha_{1 / r}$ on the domain of $\theta$ with the action of $\alpha$ on the range of $\theta$. First note that (2.3.25) is equivariant with respect to the natural actions of $\alpha_{1 / r}$ on both sides. We claim 
that the map (2.3.29) identifies the action of $\alpha_{1 / r}$ on its domain with the action of $\alpha$ on its range. To see this it suffices to work at the level of sections. We shall use the notation (sheaves: $\mathcal{F}, \mathcal{G}$; open-sets: $U, V$; sections: $f, g$ ) introduced in the discussion leading up to the definition of the map (2.3.29).

Now $\alpha_{1 / r}$ acts on $f \in \mathcal{F}(U)$ via

$$
\left(\alpha_{1 / r} \cdot f\right)(z):=\alpha_{1 / r}^{-1} \cdot f\left(\alpha_{1 / r}(z)\right) .
$$

On the other hand $\alpha$ acts on $g \in \mathcal{G}(V)$ via

$$
(\alpha \cdot g)(E, \sigma)=p^{*} g(\alpha(E, \sigma))
$$

where $p^{*}: \mathrm{H}^{1}\left(E^{\prime}, \mathbb{R}\right) \rightarrow \mathrm{H}^{1}(E, \mathbb{R})$ is pull-back with respect to the projection $p: E \rightarrow$ $E^{\prime}$. Now suppose that $f \leftrightarrow g$ in the dictionary (2.3.26). We show that $\alpha_{1 / r} \cdot f \leftrightarrow \alpha \cdot g$ in this dictionary. By definition $g(E, \sigma)=f(z)$ where $(E, \sigma) \sim\left(E_{z}, \gamma \sigma_{z}\right)$ for $z \in H$ and $\gamma \in \Gamma_{n}^{2}$. Recall that $\alpha(E, \sigma)=\frac{1}{s} \sum\left(E^{\prime},\left(\gamma_{\sigma} \sigma\right)^{\prime}\right)$ by (2.3.13). The $s$ level structures on $E^{\prime}$ differ from each other by elements of $\Gamma_{n}^{2}$. It follows from the $\Gamma_{n}^{2}$ equivariance of $g$ that $(\alpha \cdot g)(E, \sigma)=p^{*} g\left(E^{\prime}, \sigma^{\prime}\right)$ where $\sigma^{\prime}$ is any one of these level structures. Moreover since $E \sim E_{z}$ we have $E^{\prime} \sim E_{z+1 / r}$, and therefore $p^{*}$ may be considered as a map $p^{*}: \mathrm{H}^{1}\left(E_{z+1 / r}\right) \rightarrow \mathrm{H}^{1}\left(E_{z}\right)$. A short computation now shows that the following diagram commutes

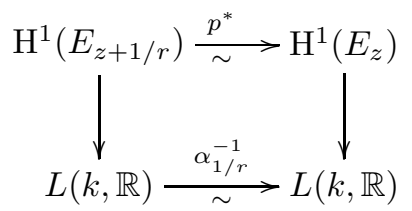

where the vertical maps are the isomorphisms (2.3.27) for $z+1 / r$ and $z$ respectively. Thus $(\alpha \cdot g)(E, \sigma)=p^{*} g\left(E^{\prime}, \sigma^{\prime}\right)=p^{*} g\left(E_{z+1 / r}, \sigma^{\prime}\right)=\alpha_{1 / r}^{-1} \cdot f(z+1 / r)$ where the last equality follows from the commutativity of the square above. On the other hand if $g^{\prime}$ corresponds to $\alpha_{1 / r} \cdot f$ in the dictionary (2.3.26) then $g^{\prime}(E, \sigma)=\alpha_{1 / r}^{-1} \cdot f\left(\alpha_{1 / r}(z)\right)$. It follows that $g^{\prime}=\alpha \cdot g$ as desired. This proves the claim.

It follows from the discussion above that the map (2.3.30) identifies the action of $\alpha_{1 / r}$ on $\mathrm{H}^{1}\left(\Gamma_{2}(n) \backslash \mathcal{H}, \tilde{L}(k, \mathbb{R})\right)$ with the action of $\alpha$ on $\mathrm{H}^{1}\left(M_{n^{2}}, \operatorname{Sym}^{k} R^{1} \pi_{*} \mathbb{R}\right)^{\Gamma_{n}^{2}}$. Let $\theta$ be the isomorphism in (2.3.31). Then since $\alpha_{1 / r}$ and $\alpha$ preserve the respective parabolic parts of the above cohomology groups, we see that the diagram

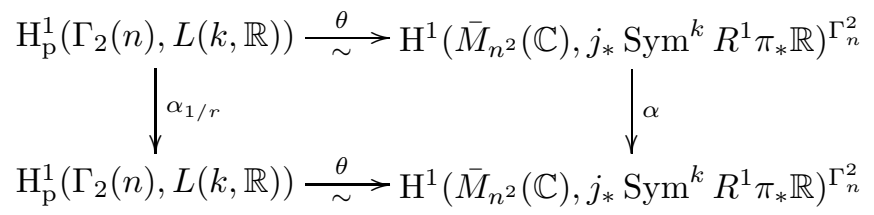

commutes, as desired.

Finally we consider the equivariance of $\alpha$ with respect to the isomorphism of Scholl. By the Leray spectral sequence for the cover $\pi_{k}: X_{n^{2}}^{k} \rightarrow M_{n^{2}}$ and the Künneth formula one has an isomorphism

$$
\mathrm{H}^{*}\left(X_{n^{2}}^{k}, \mathbb{Q}\right)(\varepsilon) \stackrel{\sim}{\longrightarrow} \mathrm{H}^{1}\left(M_{n^{2}}, \operatorname{Sym}^{k} R^{1} \pi_{*} \mathbb{Q}\right)
$$


By the functoriality of the Leray spectral sequence the following diagram commutes:

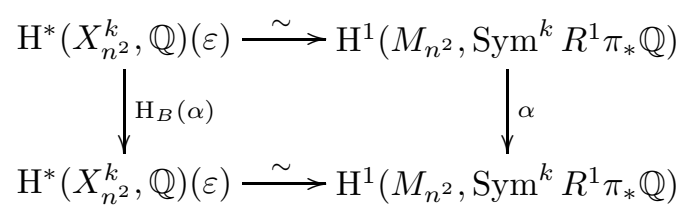

Since

$$
\begin{aligned}
\mathrm{H}_{B}\left(W_{n^{2}}\right) & \subset \mathrm{H}^{*}\left(X_{n^{2}}^{k}, \mathbb{Q}\right)(\varepsilon), \\
\mathrm{H}^{1}\left(\bar{M}_{n^{2}}(\mathbb{C}), j_{*} \operatorname{Sym}^{k} R^{1} \pi_{*} \mathbb{Q}\right) & \subset \mathrm{H}^{1}\left(M_{n^{2}}, \operatorname{Sym}^{k} R^{1} \pi_{*} \mathbb{Q}\right),
\end{aligned}
$$

and $\mathrm{H}_{B}(\alpha)$ and $\alpha$ respectively preserve these smaller spaces the above square induces the following commutative diagram

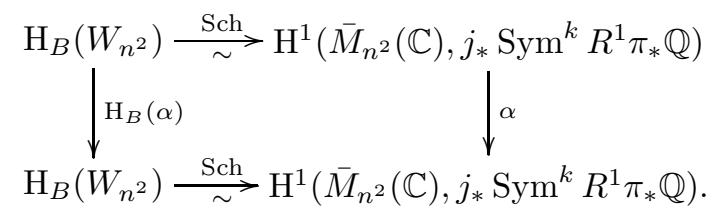

Finally taking $\Gamma_{n}^{2}$ invariants and tensoring with $\mathbb{R}$ shows the diagram

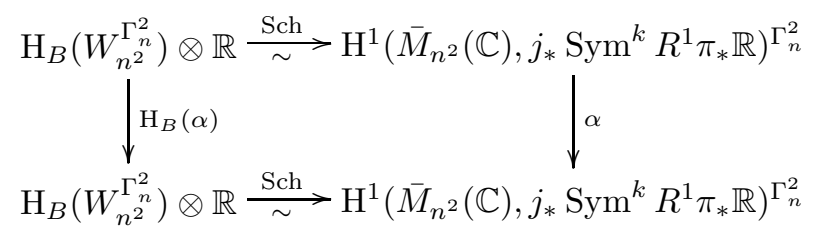

commutes.

The lemma now follows from the commutativity of the diagrams (2.3.33), (2.3.34) and (2.3.35).

Consider the commutative diagram with exact bottom row

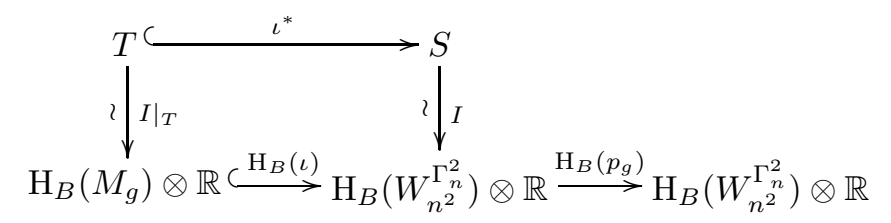

where $p_{g}: W_{n^{2}}^{\Gamma_{n}^{2}} \rightarrow W_{n^{2}}^{\Gamma_{n}^{2}}$ is a projector whose kernel is the sub-object morphism $\iota: M_{g} \rightarrow W_{n^{2}}^{\Gamma_{n}^{2}}$ and $\iota^{*}$ is the inclusion map $T \hookrightarrow S$.

Let $\tilde{\eta}_{\gamma}: M_{g} \rightarrow W_{n^{2}}^{\Gamma_{n}^{2}}$ be defined by

$$
\tilde{\eta}_{\gamma}:=\sum_{u} \alpha^{u} \circ \iota \circ \chi_{\gamma}^{-1}(u)
$$

where $\alpha^{u}$ denotes the $u$-fold composition of $\alpha: W_{n^{2}}^{\Gamma_{n}^{2}} \rightarrow W_{n^{2}}^{\Gamma_{n}^{2}}$. Then by the lemma above $I$ identifies

$$
\mathrm{H}_{B}\left(\tilde{\eta}_{\gamma}\right): \mathrm{H}_{B}\left(M_{g}\right) \otimes \mathbb{R} \rightarrow \mathrm{H}_{B}\left(W_{n^{2}}^{\Gamma_{n}^{2}}\right) \otimes \mathbb{R}
$$


with

$$
\sum_{u} \alpha_{1 / r}^{u} \circ \iota^{*} \circ \chi_{\gamma}^{-1}(u)=\sum_{u} \alpha_{u / r} \circ \iota^{*} \circ \chi_{\gamma}^{-1}(u)=\iota^{*} \circ \eta_{\gamma}^{*} .
$$

Hence $\mathrm{H}_{B}\left(\tilde{\eta}_{\gamma}\right)$ factors through $\mathrm{H}_{B}(\iota)$ and therefore

$$
\mathrm{H}_{B}\left(p_{g} \circ \tilde{\eta}_{\gamma}\right)=\mathrm{H}_{B}\left(p_{g}\right) \circ \mathrm{H}_{B}\left(\tilde{\eta}_{\gamma}\right)=0 .
$$

Since the realization functor $\mathrm{H}_{B}$ is faithful on Grothendieck motives $\tilde{\eta}_{\gamma}$ factors through $\iota$ producing a morphism $\eta_{\gamma}: M_{g} \rightarrow M_{g}$ whose Betti realization $\mathrm{H}_{B}\left(\eta_{\gamma}\right)$ is via $I$ identified with $\eta_{\gamma}^{*}$.

Invoking the faithfulness of the functor $\mathrm{H}_{B}$ once again we see that the assignment $\eta_{\gamma} \mapsto \mathrm{H}_{B}\left(\eta_{\gamma}\right)$ produces an isomorphism from the sub-algebra of $\operatorname{End}\left(M_{g}\right)$ generated (over $E$ ) by the $\eta_{\gamma}$ to the sub-algebra of $\operatorname{End}\left(\mathrm{H}_{B}\left(M_{g}\right) \otimes \mathbb{R}\right.$ ) generated by the $\mathrm{H}_{B}\left(\eta_{\gamma}\right)$. The isomorphism $\left.I\right|_{T}$ then identifies this algebra with the sub-algebra of $\operatorname{End}(T)$ generated by the $\eta_{\gamma}^{*}$. But the latter is isomorphic to $X^{\mathrm{op}} \cong X$ as we have already remarked.

We have shown that there is an embedding $X \hookrightarrow X_{g}=\operatorname{End}\left(M_{g}\right)$. Theorem 2.3.8 now follows from Proposition 2.3.9.

Proof of Proposition 2.3.9. It remains to prove the proposition. As in the proof of the theorem let $k+2$ be the weight of $f$ and $n=N$ be the level. Thus $n \geq 3$ by hypothesis.

Let

$$
\Gamma_{n}^{1}=\left\{\left(\begin{array}{ll}
* & * \\
0 & 1
\end{array}\right) \in \mathrm{GL}_{2}\left(\mathbb{Z} / n^{2}\right)\right\} .
$$

Then as in Lemma 2.3.10 the map

$$
z \mapsto\left(\mathbb{C} /(\mathbb{Z}+\mathbb{Z} z), \sigma: 1 / n^{2} \mapsto\left(\begin{array}{l}
1 \\
0
\end{array}\right), z / n^{2} \mapsto\left(\begin{array}{l}
0 \\
1
\end{array}\right)\right)
$$

induces an isomorphism

$$
\Gamma_{1}(n) \backslash \mathcal{H} \stackrel{\sim}{\longrightarrow} \Gamma_{n}^{1} \backslash M_{n}(\mathbb{C}) .
$$

Now fix a divisor $d$ of $n$. There is a natural map $\tau_{d}: \Gamma_{1}\left(n^{2}\right) \backslash \mathcal{H} \rightarrow \Gamma_{1}(n) \backslash \mathcal{H}$ induced by $z \mapsto d z$. In view of the isomorphism (2.3.36) one might expect that $\tau_{d}$ has a motivic avatar. This is in fact true: we now write this map down and will denote it by $\tau(d)$. Let $(E, \sigma) \in M_{n^{2}}$. Let

$$
C_{d}=\left\langle\left(\begin{array}{c}
n^{2} / d \\
0
\end{array}\right)\right\rangle \subset\left(\mathbb{Z} / n^{2}\right)^{2}
$$

and let $H=\sigma^{-1}\left(C_{d}\right)$. Let $E^{\prime}=E / H$ and let $\sigma^{\prime}$ be the following level $n$-structure on $E^{\prime}$. Write $\bar{\sigma}: E\left[n^{2}\right] / H \stackrel{\sim}{\longrightarrow}\left(\mathbb{Z} / n^{2}\right)^{2} / C_{d}$ for the isomorphism induced by $\sigma$. Then the natural sequence of maps

$$
E^{\prime}[n] \longrightarrow E\left[n^{2}\right] / H \stackrel{\bar{\sigma}}{\sim}\left(\mathbb{Z} / n^{2}\right)^{2} / C_{d}
$$

is an isomorphism onto the $n$-torsion of $\left(\mathbb{Z} / n^{2}\right)^{2} / C_{d}$. Since this $n$-torsion subgroup is also the isomorphic image of $(\mathbb{Z} / n)^{2}$ under the map $(\mathbb{Z} / n)^{2} \hookrightarrow\left(\mathbb{Z} / n^{2}\right)^{2} / C_{d}$ induced by $\left(\begin{array}{l}x \\ y\end{array}\right) \mapsto\left(\begin{array}{c}n x / d \\ n y\end{array}\right)$ the above sequence of maps induces an isomorphism

$$
E^{\prime}[n] \stackrel{\sim}{\longrightarrow}(\mathbb{Z} / n)^{2}
$$

which we shall define to be $\sigma^{\prime}$. Finally we define $\tau(d): M_{n^{2}} \rightarrow M_{n}$ via $\tau(d)(E, \sigma)=$ $\left(E^{\prime}, \sigma^{\prime}\right)$. 
One can check (cf. Lemma 2.3.14) that $\tau(d)$ induces a map $\tau(d): \Gamma_{n^{2}}^{1} \backslash M_{n^{2}} \rightarrow$ $\Gamma_{n}^{1} \backslash M_{n}$ and that (cf. Lemma 2.3.16) the following diagram commutes:

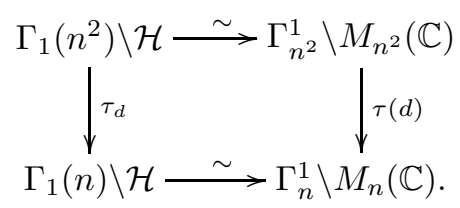

We now extend the definition of $\tau(d)$ to a correspondence $\tau(d): X_{n^{2}} \rightarrow X_{n}$ as follows:

$$
\tau(d):(e \in E, \sigma) \mapsto \frac{1}{n^{2}} \sum_{t \in E\left[n^{2}\right]}\left(p(e)+p(t) \in E^{\prime},(\sigma)^{\prime}\right)
$$

where $p: E \rightarrow E^{\prime}$ is the canonical projection. This correspondence naturally extends to a correspondence $\tau(d): \bar{X}_{n^{2}}^{k} \rightarrow \bar{X}_{n}^{k}$. A short check (cf. Lemma 2.3.21) shows that this last map is equivariant with respect to the projectors $\Pi_{\varepsilon}$ and it quasi-commutes (cf. Lemma 2.3.22) with the respective actions of $\Gamma_{n^{2}}^{1}$ and $\Gamma_{n}^{1}$ on $\bar{X}_{n^{2}}^{k}$ and $\bar{X}_{n}^{k}$. Write $W_{1}(n)$ for $W_{n}^{\Gamma_{n}^{1}}$ and $W_{1}\left(n^{2}\right)$ for $W_{n^{2}}^{\Gamma_{n^{2}}^{1}}$. The above remarks imply that $\tau(d)$ induces a morphism of motives:

$$
\tau(d): W_{1}(n) \rightarrow W_{1}\left(n^{2}\right) .
$$

The arrow is reversed since we are now in the category of motives. Let $\mathrm{H}_{B}(\tau(d))$ : $\mathrm{H}_{B}\left(W_{1}(n)\right) \rightarrow \mathrm{H}_{B}\left(W_{1}\left(n^{2}\right)\right)$ denote the induced map on Betti realizations.

The map $z \mapsto d z$ also induces a map $\tau_{d}^{*}: S_{k}\left(\Gamma_{1}(n)\right) \rightarrow S_{k}\left(\Gamma_{1}\left(n^{2}\right)\right)$ mapping $h(z)$ to $h(d z)$ for $h \in S_{k}\left(\Gamma_{1}(n)\right)$. Then the analogs of the isomorphism $I$, namely:

$$
\begin{aligned}
S_{k}\left(\Gamma_{1}(n)\right) & \stackrel{\sim}{\longrightarrow} \mathrm{H}_{B}\left(W_{1}(n)\right) \otimes \mathbb{R} \\
S_{k}\left(\Gamma_{1}\left(n^{2}\right)\right) & \stackrel{\sim}{\longrightarrow} \mathrm{H}_{B}\left(W_{1}\left(n^{2}\right)\right) \otimes \mathbb{R}
\end{aligned}
$$

identify the action of $\tau_{d}^{*}$ with the action of $\mathrm{H}_{B}(\tau(d))$ (cf. Lemma 2.3.32).

Now let $\tilde{\tau}: \sum_{d \mid n} \mu(d) \tau(d) \circ a_{d}: M_{f} \rightarrow W_{1}\left(n^{2}\right)$. Let $T_{f}$ respectively $T_{g}$ be the subspaces of $S_{k}\left(\Gamma_{1}(n)\right)$ respectively $S_{k}\left(\Gamma_{1}\left(n^{2}\right)\right)$ spanned by $f$ respectively $g$ and their conjugates. Let $\tilde{\tau}^{*}: T_{f} \rightarrow S_{k}\left(\Gamma_{1}\left(n^{2}\right)\right)$ be the map induced by $\tilde{\tau}$ on realizations. Since $\tilde{\tau}^{*}$ maps $T_{f}$ isomorphically to $T_{g}$, arguments similar to those occurring towards the end of the proof of the theorem above show that $\tilde{\tau}$ in fact factors through $\iota: M_{g} \rightarrow W_{1}\left(n^{2}\right)$. That is, there is a map $\tau: M_{f} \rightarrow M_{g}$ such that $\tilde{\tau}=\iota \circ \tau$.

We now claim that $\tau: M_{f} \rightarrow M_{g}$ is an isomorphism. The following argument was suggested to us by D. Blasius. Let $\tau^{t}: M_{g}^{t} \rightarrow M_{f}^{t}$ denote the transpose of $\tau$. Let $w=k+1$ denote the common weight of the pure motives $M_{f}$ and $M_{g}$. Let $a: M_{f} \stackrel{\sim}{\longrightarrow} M_{f}^{t}(-w)$ and $b: M_{g} \stackrel{\sim}{\longrightarrow} M_{g}^{t}(-w)$ be polarizations of $M_{f}$ and $M_{g}$. Let $\rho=a^{-1} \circ \tau^{t}(-w) \circ b: M_{g} \rightarrow M_{f}$. Since $\tau$ is an isomorphism on realizations, $\rho$ is as well. In particular $h=\rho \circ \tau: M_{f} \rightarrow M_{f}$ is an isomorphism on realizations. Let $\mathrm{H}_{B}(h)$ be the map induced by $h$ on $M_{B}=\mathrm{H}_{B}\left(M_{f}\right)$. Let $P(x) \in \mathbb{Q}[x]$ be the minimal polynomial of $\mathrm{H}_{B}(h)$. Suppose the constant term of $P(x)$ is 0 , that is suppose that $P(x)=x P_{1}(x)$ for some polynomial $P_{1}(x) \in \mathbb{Q}[x]$. Then $\mathrm{H}_{B}(h) P_{1}\left(\mathrm{H}_{B}(h)\right)=0$. Since $\mathrm{H}_{B}(h)$ is an isomorphism we have $P_{1}\left(\mathrm{H}_{B}(h)\right)=0$, and therefore as usual, 
$P_{1}(h)=0$. This contradicts the minimality of $P$ so the constant term of $P$ is nonzero. It follows that $h^{-1}$ is a polynomial of degree $\operatorname{deg}(P)-1$ in $h$. The morphism $h^{-1} \circ \rho$ is then the inverse of $\tau$.

It follows from the claim that $X_{f} \cong X_{g}$. This proves the proposition.

2.4. Tate conjecture for $M_{f}$. In [Rib80, Theorem 6.1] Ribet shows how the Tate conjecture for the abelian variety $A_{f}$ can be deduced from Theorem 2.3.6. We now observe that Theorem 2.3.8 can be similarly used to prove the Tate conjecture for the motive $M_{f}$, for $f$ of weight $k>2$, under an injectivity assumption.

Let $\ell$ be a prime and let $M_{\ell}$ denote the $\ell$-adic realization of $M_{f}$. Let $K$ be a number field and set $G_{K}=\operatorname{Gal}(\overline{\mathbb{Q}} / K)$. Write $\operatorname{End}_{K}\left(M_{f}\right)$ for the endomorphisms of $M_{f}$ which are defined over $K$. Then there is a natural map

$$
\operatorname{End}_{K}\left(M_{f}\right) \hookrightarrow \operatorname{End}_{G_{K}}\left(M_{\ell}\right)
$$

which is tautologically injective since morphisms in the category of motives are defined modulo cohomological equivalence. Let

$$
\alpha_{K}: \operatorname{End}_{K}\left(M_{f}\right) \otimes_{\mathbb{Q}} \mathbb{Q}_{\ell} \rightarrow \operatorname{End}_{G_{K}}\left(M_{\ell}\right)
$$

denote the scalar extension of the map (2.4.1) to $\mathbb{Q}_{l}$. Unlike the abelian variety case it is not known that $\alpha_{K}$ is injective. The Tate conjecture for $M_{f}$ says that $\alpha_{K}$ is an isomorphism.

Corollary 2.4.2. Assume that $\alpha_{K}$ is injective for some number field $K$ containing the fields of definitions of the endomorphisms of $M_{f}$. Then

(1) $\alpha_{K}$ is an isomorphism for every number field $K$.

(2) $X_{f}$ is isomorphic to $X$.

Proof. Let $K$ be any number field satisfying the hypothesis of the corollary. We claim that $\alpha_{K}$ is an isomorphism. By assumption $\alpha_{K}$ is injective. It follows from Theorem 2.3.8, noting $\operatorname{End}_{K}\left(M_{f}\right)=\operatorname{End}\left(M_{f}\right)=X_{f}$, that the domain of $\alpha_{K}$ has dimension at least $d:=[E: F][E: \mathbb{Q}]$ over $\mathbb{Q}_{\ell}$. On the other hand the range of $\alpha_{K}$ has dimension $d$ over $\mathbb{Q}_{\ell}$ by [Rib80, Theorem 4.4] (which does not need the assumption $k=2$ imposed there). So $\alpha_{K}$ must be an isomorphism and $X_{f}$ must be isomorphic to $X$. This proves the first statement for number fields $K$ satisfying the hypothesis of the corollary as well as the second statement. Now suppose $K$ is an arbitrary number field. Let $K_{0}$ be a sufficiently large finite Galois extension of $\mathbb{Q}$ containing $K$ as well as the fields of definition of the endomorphisms of $M_{f}$. Then $\alpha_{K_{0}}$ is injective and therefore, by what we have just shown, an isomorphism. It now follows that $\alpha_{L}$ is an isomorphism for all number fields $L$ intermediate between $K_{0}$ and $\mathbb{Q}$ by taking invariants under $\operatorname{Gal}(\overline{\mathbb{Q}} / L)$ on both sides of the $\operatorname{Gal}(\overline{\mathbb{Q}} / \mathbb{Q})$ equivariant isomorphism $\alpha_{K_{0}}$. In particular $\alpha_{K}$ is an isomorphism, proving the first statement in general.

2.5. The Brauer class of $X$. We have the following theorem which was proved by Ribet [Rib81, Theorem 1] when $k=2$.

Theorem 2.5.1. Assume $k \geq 2$. The algebra $X$ is a central simple algebra over $F$ with maximal commutative subfield $E$. Moreover the class of $X$ in $\operatorname{Br}(F)$ is 2-torsion.

Proof. This was proved at the beginning of Section 2.3. The fact that $X$ is 2torsion may also be seen as follows. This argument is similar to the one attributed 
by Ribet to Tunnell [Rib81, Theorem 1] in the case $k=2$. By Wedderburn's theorem $X \cong M_{n}(C)$ for some positive integer $n$ and some central simple division algebra $C$ over $F$. Now let $M_{B}$ denote the Betti realization of $M_{f} . M_{B}$ is vector space of dimension 2 over $E$, and therefore of dimension $2[E: F]$ over $F$. $X$ acts on all the realizations of $M_{f}$, so in particular $X$ acts on $M_{B}$. Thus $C$ acts on $M_{B}$ as well, and the dimension of $M_{B}$ as a $C$-module must be a multiple of $n$. Since the dimension of $C$ over $F$ is $([E: F] / n)^{2}$ we have

$$
\begin{aligned}
2[E: F] & =\operatorname{dim}_{F}\left(M_{B}\right)=\operatorname{dim}_{F}(C) \cdot \operatorname{dim}_{C}\left(M_{B}\right) \\
& =[E: F] \cdot \frac{[E: F]}{n} \cdot \frac{\operatorname{dim}_{C}\left(M_{B}\right)}{n} .
\end{aligned}
$$

In particular $[E: F] / n=1$ or 2 . This proves that $X$ is 2 -torsion in $\operatorname{Br}(F)$.

An immediate consequence of the above theorem is that $X$ is either

- a matrix algebra over $F$, or,

- a matrix algebra over a central simple quaternion division algebra over $F$.

In [Rib80] Ribet remarks that it seems hard to distinguish the above two cases by 'pure thought'. Ribet was concerned with the case $k=2$ but his remark is equally relevant in the higher weight case.

In [Rib81] Ribet gives two different sufficient conditions for $X$ to be a matrix algebra over $F$ when $k=2$. The second of these requires $A_{f}$ to have potentially multiplicative reduction at at least one place of $\mathbb{Q}[$ Rib81, Theorem 3]. A suitable generalization of this result holds when $k \geq 2$ under the additional assumption that $k$ is even. Since our proof of this fact has a local flavour we will defer discussing it to the next section (see Theorem 3.4.6 below).

Ribet's first sufficient condition for $X$ to be a matrix algebra over $F$ requires that all endomorphisms of $A_{f}$ are defined over $\mathbb{R}$ (see [Rib81, Theorem 2]). This has the following generalization to higher weight. We recall some facts. Let $M_{B}$ denote the Betti realization of $M_{f}$. Recall that $M_{B}$ is a vector space of dimension 2 over $E$. Let

$$
F_{\infty}: M_{B} \rightarrow M_{B}
$$

be the $E$-linear involution on $M_{B}$ induced by the action of complex conjugation on $\mathrm{H}^{*}\left(\overline{\bar{X}}_{n^{2}}^{k}(\mathbb{C}), \mathbb{Q}\right)$. We have:

Theorem 2.5.2. Assume that each endomorphism of $X$, thought of as endomorphism of $M_{B}$, commutes with $F_{\infty}$. Then $X$ is a matrix algebra over $F$.

Proof. There is a decomposition of $E \otimes \mathbb{C}$-modules

$$
M_{B} \otimes \mathbb{C}=M_{B}^{0, k-1} \oplus M_{B}^{k-1,0} .
$$

Since $F_{\infty} \otimes 1$ permutes the two spaces occurring on the right hand side of (2.5.3) the eigenspaces of $F_{\infty}$ on $M_{B}$ both have dimension $[E: F]$ over $F$. By hypothesis $X \cong M_{n}(C)$ preserves these eigenspaces. Hence $M_{B}^{+}$, the +-eigenspace of $F_{\infty}$ on $M_{B}$, is an $X$ module, and therefore a $C$-module. As above this implies $\operatorname{dim}_{C}\left(M_{B}^{+}\right)$ is a multiple of $n$ and we have

$$
\begin{aligned}
{[E: F] } & =\operatorname{dim}_{F}\left(M_{B}^{+}\right)=\operatorname{dim}_{F}(C) \cdot \operatorname{dim}_{C}\left(M_{B}^{+}\right) \\
& =[E: F] \cdot \frac{[E: F]}{n} \cdot \frac{\operatorname{dim}_{C}\left(M_{B}^{+}\right)}{n} .
\end{aligned}
$$

This forces $[E: F] / n=1$ and $C=F$ proving the theorem. 
Corollary 2.5.4. If $\chi_{\gamma}(-1)=1$ for all $\gamma \in \Gamma$ then $X$ is a matrix algebra over $F$.

Proof. The construction of Ribet [Rib81, pages 268-269] works in all weights showing that if $\chi_{\gamma}(-1)=1$ for all $\gamma \in \Gamma$ then all the endomorphisms in $X$ are defined over the real numbers. Thus the hypothesis of Theorem 2.5.2 is satisfied.

Remark 2.5.5. When $k$ is odd there is always an endomorphism in $X$ which does not commute with $F_{\infty}$. This can be seen for instance by Theorem 3.1.1 below. Thus Theorem 2.5.2 is essentially a theorem for even weight forms.

\section{Local Behaviour of $X$}

We now turn our attention to the local behaviour of the algebra $X$. Let $v$ be a place of $F$ which is either finite or infinite. Let $X_{v}=X \otimes_{F} F_{v}$. The standard exact sequence

$$
0 \rightarrow \operatorname{Br}(F) \rightarrow \bigoplus_{v} \operatorname{Br}\left(F_{v}\right) \rightarrow \mathbb{Q} / \mathbb{Z} \rightarrow 0 .
$$

shows that the Brauer class of $X$ is completely determined by the Brauer classes of the $X_{v}$. By Theorem 2.5.1 the class of $X_{v}$ in $\operatorname{Br}\left(F_{v}\right)$ is 2-torsion, so that a priori $X_{v}$ is a matrix algebra over $F_{v}$ or a matrix algebra over a division quaternion algebra over $F_{v}$.

3.1. Infinite places. As we have already remarked in the proof of Theorem 2.3.8 the number field $F$ is totally real. Thus if $v$ is an infinite place of $F$ it must be real. The following theorem due to Momose investigates the structure of $X_{v}$ for such places.

Theorem 3.1.1. Let $v$ be a (real) infinite place of $F$. If $k$ is even then $X_{v}$ is a matrix algebra over $\mathbb{R}$. If $k$ is odd then $X_{v}$ is a matrix algebra over the quaternion division algebra $\mathbb{H}$ of Hamiltonians.

Proof. This follows easily from [Mom81, Theorem 3.1 ii)]. Indeed since $X$ acts faithfully on $M_{B}$ we may consider $X$ as a sub-algebra of $\operatorname{End}\left(M_{B}\right)$. Let $Z$ be the centralizer of $X$ in $\operatorname{End}_{F}\left(M_{B}\right)$. Then $Z$ is a central simple algebra over $F$ and $[X: F][Z: F]=\left[\operatorname{End}_{F}\left(M_{B}\right): F\right]$ (so $Z$ has $F$-dimension 4 ). In particular the natural multiplication map $X \otimes_{F} Z \rightarrow \operatorname{End}_{F}\left(M_{B}\right)$ is an isomorphism (it is injective since the domain is simple, and surjective because of dimension reasons). It follows that the Brauer class of $X$ is the inverse of the Brauer class of $Z$. But Momose has shown [Mom81, Theorem 3.1 ii)] that $Z$ is totally indefinite if and only if $k$ is even. The theorem follows.

3.2. Modular crystals. In the next two sections we will investigate the structure of $X_{v}$ at the finite places $v$ of $F$. In this section we prepare the ground by studying the crystalline realization of $M_{f}$ at $p$ where $p$ is the residue characteristic of $v$.

It is convenient to recall some general terminology. Let $K$ be an extension of $\mathbb{Q}_{p}$ with residue field $\mathbb{F}$. Let $K_{0}$ denote the maximal unramified extension of $\mathbb{Q}_{p}$ in $K$. Recall that a filtered $(\phi, N)$-module $D$ over $K$ is a finite dimensional $K_{0}$-vector space $D$ endowed with

- a $\sigma$-linear Frobenius map $\phi: D \rightarrow D$,

- a linear map $N: D \rightarrow D$ such that $N \phi=p \phi N$, and,

- a decreasing filtration $\mathrm{Fil}^{i}\left(D_{K}\right)$ on $D_{K}=D \otimes K$ such that $\mathrm{Fil}^{i}\left(D_{K}\right)=D_{K}$ for $i<<0$ and $\mathrm{Fil}^{i}\left(D_{K}\right)=0$ for $i>>0$. 
We say that $D$ has coefficients in $E$ if $D$ is a free $E \otimes K_{0}$-module such that the action of $E$ commutes with all the structures.

Given $f$ and a prime $w \mid p$ of $E$ there is a way to attach a filtered $(\phi, N)$-module over $K$ for some extension $K$ of $\mathbb{Q}_{p}$ as follows.

Let $\rho_{f}: \operatorname{Gal}(\overline{\mathbb{Q}} / \mathbb{Q}) \rightarrow \mathrm{GL}_{2}\left(E_{w}\right)$ denote the $w$-adic Galois representation attached to $f$. It is known that $\left.\rho_{f}\right|_{G_{p}}$ is potentially semi-stable. Fix an extension $K$ of $\mathbb{Q}_{p}$ such that $\rho_{f}$ becomes semi-stable when restricted to $G_{K}$. Fontaine has constructed a functor $D_{\text {st }}: V \mapsto\left(B_{\mathrm{st}} \otimes_{\mathbb{Q}_{p}} V\right)^{G_{K}}$ from the category of semi-stable representations of $G_{K}$ to the category of filtered $(\phi, N)$-modules over $K$. Let $D=D_{\mathrm{st}}\left(\left.\rho_{f}\right|_{G_{K}}\right)$. This is the filtered $(\phi, N)$-module attached to $f$ (and $p$ and $K$ ). It is a free module over $E_{w} \otimes_{\mathbb{Q}_{p}} K_{0}$ of rank 2. When $K / \mathbb{Q}_{p}$ is Galois then $D_{K}$ comes equipped with an action of its Galois group $\operatorname{Gal}\left(K / \mathbb{Q}_{p}\right)$. Write $D^{*}$ for the filtered $(\phi, N)$-module corresponding to the representation which is dual to $\left.\rho_{f}\right|_{G_{K}}$.

We now write down the filtered module $D_{w}^{*}$ attached to $f$ for various primes $w \mid p$ of $E$ in as many cases as are known following [Bre01]. We shall denote the basis of the filtered modules below of rank 2 over $E_{w}^{\prime} \otimes K_{0}$ by $e_{1}, e_{2}$ where $E_{w}^{\prime}$ is a finite extension of $E_{w}$.

Let us start with the case $p \nmid N$. In this case it turns out that $K=K_{0}=\mathbb{Q}_{p}$. Let $\mu_{1}, \mu_{2} \in E_{w}^{\prime}$ be defined by

$$
\begin{aligned}
\mu_{1} \mu_{2} & =\epsilon(p) \\
p^{k-1} \mu_{1}+\mu_{2} & =a_{p} .
\end{aligned}
$$

If $w\left(a_{p}\right)=0$ then $D_{w}^{*}$ is one of the following two filtered $(\phi, N)$-modules:

$$
D_{\text {split }}:\left\{\begin{array}{l}
\phi\left(e_{1}\right)=p^{k-1} \mu_{1} e_{1} \\
\phi\left(e_{2}\right)=\mu_{2} e_{2} \\
\operatorname{Fil}^{k-1}(D)=E_{w}^{\prime} \cdot e_{1} \\
N=0
\end{array} \quad D_{\text {non-split }}:\left\{\begin{array}{l}
\phi\left(e_{1}\right)=p^{k-1}\left(\mu_{1} e_{1}+e_{2}\right) \\
\phi\left(e_{2}\right)=\mu_{2} e_{2} \\
\mathrm{Fil}^{k-1}(D)=E_{w}^{\prime} \cdot e_{1} \\
N=0 .
\end{array}\right.\right.
$$

It is known that when $f$ is a CM-form then $D_{w}^{*}=D_{\text {split }}$. When $f$ does not have $\mathrm{CM}$ then it is expected but not known that $D_{w}^{*}=D_{\text {non-split. }}$. On the other hand if $w\left(a_{p}\right)>0$ then $D_{w}^{*}$ is

$$
D_{\text {non-ordinary }}:\left\{\begin{array}{l}
\phi\left(e_{1}\right)=p^{k-1} \epsilon(p) e_{2} \\
\phi\left(e_{2}\right)=-e_{1}+a_{p} e_{2} \\
\operatorname{Fil}^{k-1}(D)=E_{w}^{\prime} \cdot e_{1} \\
N=0 .
\end{array}\right.
$$

Now let us assume that $p \mid N$. Let $N_{p}$ be the exact power of $p$ that divides $N$. Let $C$ denote the conductor of $\epsilon$ and let $C_{p}$ denote the exact power of $p$ that divides $C_{p}$. Note $N_{p} \geq C_{p}$. It is useful to consider three cases.

(1) $N_{p}=C_{p}$ : in which case $\left|a_{p}\right|=p^{(k-1) / 2}$ (Ramified principal series),

(2) $N_{p}=1$ and $C_{p}=0$ : in which case $a_{p}^{2}=\epsilon(p) p^{k-2}$ (Steinberg),

(3) $N_{p} \neq C_{p}$ and $N_{p} \geq 2$ in which case $a_{p}=0$ (Other).

In the first case the local component at $p$ of the automorphic representation is in the ramified principal series whereas in the second case it is (the twist by an unramified character of $\mathbb{Q}_{p}$ of) the Steinberg representation. The third case includes the cases when this representation is supercuspidal (or extraordinary when $p=2$ ). 
We discuss each of these cases in turn. Assume that we are in the first case. Then it is known that we may take $K$ to be the totally ramified (but tamely ramified) extension of $\mathbb{Q}_{p}$ given by adjoining a primitive $p^{\text {th }}$ root of $1: K=\mathbb{Q}_{p}\left(\mu_{p}\right)=\mathbb{Q}_{p}(x)$ where $x^{p-1}=-p$. In particular we see that $K_{0}=\mathbb{Q}_{p}$ again. Define $\epsilon^{\prime}$ to be the prime-to- $p$ part of $\epsilon$. Let $\omega:(\mathbb{Z} / p)^{\times} \rightarrow \mu_{p-1}$ denote the Teichmüller character. Define the integer $i$ by $1 \leq i \leq p-2$ and $\epsilon=\omega^{i} \cdot \epsilon^{\prime}$. Now assume that $N_{p}=C_{p}=1$. Then $D_{w}^{*}$ is given by

$$
D_{\text {ram-prin-series }}:\left\{\begin{array}{l}
\phi\left(e_{1}\right)=a_{p} e_{1} \\
\phi\left(e_{2}\right)=\epsilon^{\prime}(p) \bar{a}_{p} e_{2}=\left(\epsilon^{\prime}(p) p^{k-1} / a_{p}\right) e_{2} \\
\operatorname{Fil}^{k-1}\left(D_{K}\right)=\left(K \otimes E_{w}\right) \cdot\left(e_{1}+x^{-i} e_{2}\right) \\
N=0 \\
g\left(e_{1}\right)=e_{1} \\
g\left(e_{2}\right)=\omega(g)^{i} e_{2}
\end{array}\right.
$$

where $g \in \operatorname{Gal}\left(K / \mathbb{Q}_{p}\right)$. It is likely that $D_{w}^{*}$ has the same shape even when $N_{p}=$ $C_{p}>1$ except that the filtration is probably more complicated to write down.

Now assume that we are in the second case (the Steinberg case). Then it is known that we may take $K=K_{0}=\mathbb{Q}_{p}$. Let $\mathcal{L} \in E_{w}$ denote the so called $\mathcal{L}$-invariant of $f$. Then $D_{w}^{*}$ is given by

$$
D_{\text {Steinberg }}:\left\{\begin{array}{l}
\phi\left(e_{1}\right)=p a_{p} e_{1} \\
\phi\left(e_{2}\right)=a_{p} e_{2} \\
\operatorname{Fil}^{k-1}(D)=E_{w}^{\prime} \cdot\left(e_{1}-\mathcal{L} e_{2}\right) \\
N\left(e_{1}\right)=e_{2} \\
N\left(e_{2}\right)=0 .
\end{array}\right.
$$

It would be interesting to write down what $D_{w}^{*}$ is in the third case. As already mentioned, in [Bre01] Breuil has asked for a complete general classification of the filtered modules associated to cusp forms.

Now assume that $p$ is arbitrary again. Let $M_{\text {crys }}=\oplus_{w \mid p} D_{w}$ where $w$ varies over all the places of $E$ denote the crystalline realization of the motive $M_{f}$. It is a free $E \otimes \mathbb{Q}_{p}$-module of rank 2 .

3.3. Good places. We now investigate the structure of $X_{v}$ when $v$ is a finite place of $F$, say of residue characteristic $p$. In this section we shall assume that $p \nmid N$ and shall say that $v$ is a 'good' place of $F$.

Following Ribet we shall say that $v$ is 'ordinary for $f$ ' if $p \nmid N$ and $v\left(a_{p}^{2} \epsilon(p)^{-1}\right)=$ 0 (equivalently $w\left(a_{p}\right)=0$ for one, equivalently all, places $w$ of $E$ lying over $v$ ). The following result shows that $X_{v}$ is a matrix algebra over $F$ for such $v$. It was proved by Ribet [Rib81, Theorem 6] in the case $k=2$.

Theorem 3.3.1. If $p$ is a prime not dividing $N$ and $v$ is a place of $F$ over $p$ such that $v\left(a_{p}^{2} \epsilon(p)^{-1}\right)=0$ then $X_{v}$ is a matrix algebra over $F_{v}$.

Proof. Ribet's proof in the case $k=2$ uses the $p$-adic Tate module of $\tilde{A}_{f}$, the abelian variety over $\mathbb{F}_{p}$ which is the reduction of the abelian variety $A_{f}$ at $p$. A proof when $k=2$ may be given by studying the crystal attached to $\tilde{A}_{f}$ instead. This proof generalizes to arbitrary weight $k \geq 2$ as follows. 
Let $M_{\text {crys }}$ be the crystalline realization of $M_{f}$. It is a free $E \otimes \mathbb{Q}_{p}$-module of rank two equipped with a crystalline Frobenius endomorphism $\phi$. Hence it is a crystal over $\mathbb{Q}_{p}$ of $\operatorname{rank} 2[E: \mathbb{Q}]$. As can be seen from the previous section the characteristic polynomial of $\phi\left(\right.$ over $\left.E \otimes \mathbb{Q}_{p}\right)$ is

$$
H(x):=x^{2}-a_{p} x+\epsilon(p) p^{k-1} .
$$

Let $M_{\text {crys }, v}:=M_{\text {crys }} \otimes_{F \otimes \mathbb{Q}_{p}} F_{v}$. It is a sub-crystal of $M_{\text {crys }}$ of dimension $2[E$ : $F]$ over $F_{v}$ and hence dimension $2 d$ over $\mathbb{Q}_{p}$ where $d:=[E: F]\left[F_{v}: \mathbb{Q}_{p}\right]$. The characteristic polynomial of $\phi$ over $F_{v}$ is $\operatorname{Norm}_{E \mid F}(H(x))$; over $\mathbb{Q}_{p}$ it is therefore

$$
H_{v}(x):=\operatorname{Norm}_{F_{v} \mid \mathbb{Q}_{p}}\left(\operatorname{Norm}_{E \mid F}(H(x))\right)=\prod_{w \mid v} \operatorname{Norm}_{E_{w} \mid \mathbb{Q}_{p}}(H(x))
$$

where $w$ runs through the places of $E$ lying over $v$.

If $w \mid v$ then $w\left(a_{p}\right)=0$ by the hypothesis on $v$; hence $H(x)$ has two roots in $E_{w}$, one of zero $w$-adic valuation and the other non-zero. Therefore exactly half (counted with multiplicities) of the roots of $\operatorname{Norm}_{E_{w} \mid \mathbb{Q}_{p}}(H(x))$ are w-adic units. Letting $w$ vary among all places of $E$ lying over $v$ we have that exactly $d$ of the Newton slopes of $M_{v}$ are 0 .

Let $\bar{M}_{\text {crys }, v}:=M_{\text {crys }, v} \otimes_{\mathbb{Q}_{p}} \mathbb{Q}_{p}^{\text {ur }}$ where $\mathbb{Q}_{p}^{\text {ur }}$ is the maximal unramified extension of $\mathbb{Q}_{p}$. Let $C_{0}$ be the 1-dimensional crystal $\left(\mathbb{Q}_{p}^{\text {ur }}, \sigma\right)$ with $\sigma$ the Teichmüller lift of Frobenius. By the classification theory of crystals (see [Dem72]) $\bar{M}_{\text {crys }, v}$ decomposes as $\bar{M}_{\text {crys }, v} \cong C_{0}{ }^{d} \times C^{\prime}$ where $C^{\prime}$ is a crystal with no zero Newton slopes. Let $V:=\operatorname{Hom}\left(C_{0}, \bar{M}_{\text {crys }, v}\right)$. Because $\operatorname{Hom}\left(C_{0}, C^{\prime}\right)=0$ (see [Dem72]) we have $V \cong$ $\operatorname{Hom}\left(C_{0}, C_{0}\right)^{d} \cong \mathbb{Q}_{p}{ }^{d}$.

The functor $M_{\text {crys }}$ is covariant, giving a left action of $X_{v}$ on $\mathrm{H}_{\text {crys }}^{1}\left(M_{f} \otimes \overline{\mathbb{Q}}\right)$. On the other hand $\mathrm{H}_{\text {crys }}^{1}\left(M_{f} \otimes \overline{\mathbb{Q}}\right) \cong M_{\text {crys }} \otimes_{\mathbb{Q}_{p}} \mathbb{Q}_{p}^{\text {ur }}$ so that $X_{v}$ acts on $M_{\text {crys }} \otimes_{\mathbb{Q}_{p}} \mathbb{Q}_{p}^{\text {ur }}$ and hence on

$$
M_{\text {crys }} \otimes_{\mathbb{Q}_{p}} \mathbb{Q}_{p}^{\text {ur }} \otimes_{F \otimes \mathbb{Q}_{p}} F_{v} \cong \bar{M}_{\text {crys }, v} .
$$

Since $\operatorname{Hom}\left(C_{0}, \cdot\right)$ is also covariant we obtain a left $X_{v}$-module structure on $V$. But $\operatorname{dim}_{F_{v}} V=d /\left[F_{v}: \mathbb{Q}_{p}\right]=[E: F]$ which implies that $X_{v}$ is a matrix algebra.

We shall say that a place $v$ of $F$ of residue characteristic $p$ with $p$ prime to $N$ is 'singular for $f$ ' if $0<v\left(a_{p}^{2} \epsilon(p)^{-1}\right)<k-1$. The following theorem describes the structure of $X_{v}$ at a singular place $v$ under an additional hypothesis. This theorem is new even for $k=2$.

Theorem 3.3.2. Say that $p \nmid N$ and that $v$ is a place of $F$ lying over $p$ such that

$$
0<v\left(a_{p}^{2} \epsilon^{-1}(p)\right)<k-1
$$

Let $w$ be a place of $E$ lying over $v$. If, as a fraction in lowest terms, the denominator of $w\left(a_{p}\right)$ is odd then $X_{v}$ is a matrix algebra over $F_{v}$.

Proof. The hypothesis on $v$ implies that for each $w \mid v H(x)$ has two roots in $E_{w}$, one of $w$-adic valuation $\alpha<(k-1) / 2$ and the other of $w$-adic valuation $\beta>(k-1) / 2$. $\left(\alpha, \beta \in \mathbb{Q}\right.$.) Since $w\left(a_{p}\right)=w(\alpha)=k-1-w(\beta)$ is the same for every $w \mid v$, the Newton polygon for $H(x)$ is the same for every such $w$. Therefore $d$ of the Newton slopes of $M_{\text {crys }, v}$ are $\alpha$ and the other $d$ are $\beta$. Hence $\bar{M}_{\text {crys }, v}$ decomposes as $\bar{M}_{\text {crys }, v} \cong C_{\alpha}^{n_{\alpha}} \times C_{\beta}^{n_{\beta}}$ where $C_{\alpha}, C_{\beta}$ are simple crystals of slopes $\alpha, \beta$. We have $2 d=\operatorname{dim}_{\mathbb{Q}_{p}} M_{\text {crys }, v}=\operatorname{dim}_{\mathbb{Q}_{p}^{\text {ur }}} C_{\alpha}^{n_{\alpha}}+\operatorname{dim}_{\mathbb{Q}_{p}^{\text {ur }}} C_{\beta}^{n_{\beta}}=2 s n_{\alpha}$ where $\alpha=r / s$ is expressed 
in lowest terms. This is because $C_{\alpha}$ and $C_{\beta}$ have the same dimension $s$ over $\mathbb{Q}_{p}^{\text {ur }}$ and occur with the same multiplicity $\left(n_{\alpha}=n_{\beta}\right)$ in $\bar{M}_{\text {crys }, v}$.

Let $V:=\operatorname{Hom}\left(C_{\alpha}, \bar{M}_{\text {crys }, v}\right)$. Then $V \cong \operatorname{Hom}\left(C_{\alpha}, C_{\alpha}\right)^{n_{\alpha}}$ and

$$
\operatorname{dim}_{F_{v}} V=\frac{s^{2} n_{\alpha}}{\left[F_{v}: \mathbb{Q}_{p}\right]}=\frac{s d}{\left[F_{v}: \mathbb{Q}_{p}\right]}=s[E: F] .
$$

As we have seen $V$ has a natural structure of a left $X_{v}$-module, and by hypothesis $s$ is odd. Hence $X_{v}$ must be a matrix algebra.

Corollary 3.3.3. Let $p$ and $v$ satisfy the conditions of the theorem. If $p$ does not ramify in $E$ then $X_{v}$ is a matrix algebra over $F_{v}$.

Proof. The hypothesis on $p$ implies that $w\left(a_{p}\right)$ is an integer.

We shall say that a place $v$ of $F$ of residue characteristic $p$ with $p$ prime to $N$ is 'supersingular for $f$ ' if $v\left(a_{p}^{2} \epsilon(p)^{-1}\right) \geq k-1$. Notice that the above theorems do not treat the supersingular places. To get a better feeling of why this is so one need only look at the Newton polygon of $H_{v}(x)$, the characteristic polynomial of the crystalline Frobenius $\phi$ considered as an operator on the $\mathbb{Q}_{p}$-crystal $M_{\text {crys }, v}$.

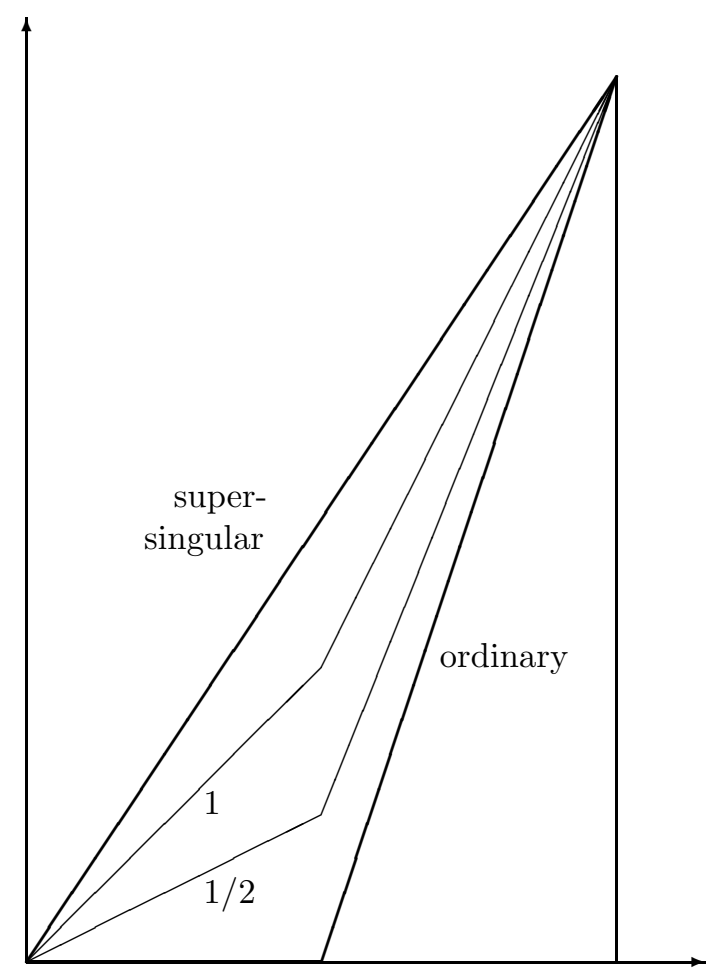

Figure 1: Newton polygons of $H_{v}(x)$.

Figure 1 depicts various possibilities for the Newton polygons of $H_{v}(x)$ (the scale is for the case $k=4)$. The lowest Newton polygon occurs when $v$ is ordinary, and corresponds to the slopes $(0, k-1)$. The top most Newton polygon occurs when $v$ is supersingular and corresponds to the slope $(k-1) / 2$. The intermediate Newton 
polygons occur when $v$ is singular and correspond to the slopes $(\alpha, k-1-\alpha)$ where $0<\alpha<(k-1) / 2$. The ordinary and singular Newton polygons are characterized by the fact that they have two distinct slopes. This can be used to advantage in studying $X_{v}$ in these cases as was done in Theorems 3.3.1 and 3.3.2. Note that Theorem 3.3.2 only treats those singular cases for which the denominator of $\alpha$ is odd. Thus for the two singular Newton polygons in Figure $1, X_{v}$ is a matrix algebra for $\alpha=1$, but may be ramified when $\alpha=1 / 2$.

3.4. Bad places. We shall say that a place $v$ of $F$ is 'bad' if the residue characteristic $p$ of $v$ divides $N$. In this section we investigate the structure of $X_{v}$ for bad $v$. We divide our discussion into the three natural cases outlined in Section 3.2. We start with the case $N_{p}=C_{p}$. We have the following theorem when $k=2$.

Theorem 3.4.1. Suppose that $p \mid N$ and that $N_{p}=C_{p}$. Assume that $k=2$. Let $v$ be a place of $F$ over $p$. Suppose that $w\left(a_{p}\right)=0$ or $w\left(\bar{a}_{p}\right)=0$ for each place $w$ of $E$ lying over $v$. Then $X_{v}$ is a matrix algebra over $F_{v}$.

Proof. The hypothesis on $p$ implies that $A_{f}$ has potentially good reduction at $p$. In fact if one takes $K$ to be the completion of the cyclotomic field $\mathbb{Q}\left(\mu_{p^{n}}\right)$, for sufficiently large $n$, at the unique prime $\mathfrak{p}$ of $K$ lying over $p$, then $B=A_{f} \times \mathbb{Q}_{p} K$ has good reduction at $\mathfrak{p}$. Let $\tilde{B}$ denote the reduction of $B$ at $\mathfrak{p}$. It is an abelian variety over $\mathbb{F}_{p}$ since the residue degree $f(\mathfrak{p} \mid p)=1$.

Let $\epsilon^{\prime}$ denote the prime to $p$ part of $\epsilon$. The characteristic polynomial of Frob acting on the $\ell$-adic Tate module of $\tilde{B}$ for $\ell \neq p$ is

$$
\operatorname{Norm}_{E \mid \mathbb{Q}}\left(\left(x-a_{p}\right)\left(x-\epsilon^{\prime}(p) \bar{a}_{p}\right)\right) .
$$

This can be seen for instance by studying the local factor at $p$ of the $L$-function of $L(s, f)$ attached to $f$.

Let $V$ denote the $p$-adic Tate module attached to $\tilde{B}$. Let $w$ be a place of $E$ lying over $v$ and let $V_{w}$ denote the $w$ eigenspace of $V$. Then the dimension of $V_{w}$ over $E_{w}$ is the number of $w$-adic unit roots of the polynomial

$$
\left(x-a_{p}\right)\left(x-\epsilon^{\prime}(p) \bar{a}_{p}\right)
$$

which by hypothesis is 1 . Let $V_{v}$ denote the $v$-eigenspace of $V$. This has dimension $[E: F]$ over $F_{v}$. Since $X_{v}$ acts on $V_{v}$ we see that $X_{v}$ must be a matrix algebra over $F_{v}$.

We now extend the above theorem to $k \geq 2$ subject to the restriction that $N_{p}=C_{p}=1$.

Theorem 3.4.2. Suppose $N_{p}=C_{p}=1$ and $k \geq 2$. Assume that $w\left(a_{p}\right)=0$ or $w\left(\bar{a}_{p}\right)=0$ for each place $w$ of $E$ lying over $v$. Then $X_{v}$ is a matrix algebra over $F_{v}$.

Proof. As already mentioned $\rho_{f}$ has semi-stable (in fact good) reduction over $G_{K}$ where $K$ is the completion of $\mathbb{Q}\left(\mu_{p}\right)$ at the unique prime lying above $p$. Let $D=$ $\oplus_{w \mid p} D_{w}$ denote the associated filtered $(\phi, N)$-module. Here $D_{w}$, the $w$-eigenspace of $D$, is the filtered $(\phi, N)$ module attached to $f$ and $w \mid p$ as in Section 3.2. A study of the crystal $D$ shows that the characteristic polynomial of $\phi$ is

$$
\operatorname{Norm}_{E \mid \mathbb{Q}}\left(\left(x-a_{p}\right)\left(x-\epsilon^{\prime}(p) \bar{a}_{p}\right)\right) .
$$


Fix $w \mid p$. When $w\left(a_{p}\right)<\frac{k-1}{2}$ we see that $D_{w}$ has Newton slopes $w\left(a_{p}\right)$ and $w\left(\bar{a}_{p}\right)$ each occurring with multiplicity $\left[E_{w}: \mathbb{Q}_{p}\right]$. After the vanishing hypothesis in the statement of the theorem we conclude that $D_{v}=\oplus_{w \mid v} D_{w}$ is an ordinary crystal of dimension $2[E: F]\left[F_{v}: \mathbb{Q}_{p}\right]$ over $\mathbb{Q}_{p}$. Now an argument similar to that used in the proof of Theorem 3.3.1 shows that $X_{v}$ is a matrix algebra over $F_{v}$.

Remark 3.4.3. We have required $N_{p}=C_{p}=1$ above, and below, simply because the reference we have used to write down the crystal attached to $f$, namely Breuil's CRM lecture notes [Bre01], requires this. It is quite possible that the theorem is true simply if $N_{p}=C_{p}$, as is the case when $k=2$ (cf. Theorem 3.4.1).

An analog of Theorem 3.3.2 is also true when $v$ is a bad prime. We have:

Theorem 3.4.4. Say that $p \mid N$ with $N_{p}=C_{p}=1$ and say that $v$ is a place of $F$ lying over $p$. Let $\alpha \in \mathbb{Q}$ be such that

$$
0<\alpha<(k-1) / 2
$$

and $\alpha$ has odd denominator. If for each place $w$ of $E$ lying over $v$ either $w\left(a_{p}\right)=\alpha$ or $\bar{w}\left(a_{p}\right)=\alpha$ then $X_{v}$ is a matrix algebra over $F_{v}$.

Proof. The proof is similar to the proof of Theorem 3.3.2 and is omitted.

We now turn our attention to the case when $N_{p}=1$ and $C_{p}=0$ so that $f$ is 'Steinberg at $p$ '. The following result was proved by Ribet [Rib81, Theorem 3] when $k=2$.

Theorem 3.4.5. Suppose that $N_{p}=1$ and $C_{p}=0$. If $k=2$ then $X_{v}$ is a matrix algebra over $F_{v}$. In fact $X$ is a matrix algebra over $F$.

We have the following generalization of Theorem 3.4.5 when $k$ is even.

Theorem 3.4.6. Let $N_{p}=1$ and $C_{p}=0$. Assume that $k \geq 2$ is even. Then $X_{v}$ is a matrix algebra over $F_{v}$. In fact $X$ is a matrix algebra over $F$.

Proof. Assume momentarily that $k=2$. In this case as we have already remarked the theorem is due to Ribet. Ribet's proof is global in nature and proceeds as follows. Let $\tilde{A}_{f}$ denote the reduction of the abelian variety $A_{f}$ at $p$. Deligne-Rapoport have shown that when $f$ is Steinberg at $p$ then $A_{f}$ has purely multiplicative reduction at $p$, that is, the connected component of $\tilde{A}_{f}$ is a torus $T$ over $\mathbb{F}_{p}$. Ribet observes that this torus has dimension $g=[E: \mathbb{Q}]$ over $\mathbb{F}_{p}$ and has a natural action of $X$. This provides a homomorphism of $X$ into $\mathrm{M}_{g}(\mathbb{Q})$. Ribet concludes that $X$ must be a matrix algebra over $F$ for the usual dimension reasons.

This global argument may have a generalization to higher weight but it would involve making sense of the reduction of the motive $M_{f}$ at $p$. Instead we give a proof that is of a local nature.

We first show that $X_{v}$ is trivial for each place $v$ of $F$ dividing $p$. Let $\rho_{f}$ denote the $p$-adic representation attached to $f$. Then $\rho_{f}$ already has semi-stable reduction over $\mathbb{Q}_{p}$. For a place $w \mid p$ of $E$ let $D_{w}$ be the associated filtered $(\phi, N)$-module as in Section 3.2. Let $D_{v}=\oplus_{w \mid v} D_{w}$ denote the associated filtered $(\phi, N)$-module. The slopes of $\phi$ on $D_{w}$ are $k / 2$ and $k / 2-1$. When $k$ is even these are distinct integers and the usual arguments apply to show that $X_{v}$ is then necessarily a matrix algebra over $F_{v}$. 
We now turn to the other finite places of $F$. Let $\ell$ be a prime different from $p$. Let

$$
\rho_{f, \ell}: \operatorname{Gal}(\overline{\mathbb{Q}} / \mathbb{Q}) \rightarrow \mathrm{GL}_{2}\left(E \otimes \mathbb{Q}_{\ell}\right)
$$

denote that $\ell$-adic representation attached to $f$. It is a theorem of Langlands (cf. [Hid00, Theorem $3.263(\mathrm{~b})]$ ) that

$$
\left.\rho_{f, \ell}\right|_{G_{p}} \sim\left(\begin{array}{cc}
\eta \nu & * \\
0 & \eta
\end{array}\right)
$$

where $\nu$ is the $\ell$-adic cyclotomic character $G_{p} \rightarrow \mathbb{Z}_{\ell}^{\times}$and $\eta$ is an unramified character which takes Frob $_{p}$ to $a_{p}$. Let $M_{\ell}$ denote the $\ell$-adic realization of $M_{f}$. Let $\lambda$ be a place of $F$ lying over $\ell$ and let $M_{\lambda}$ denote the $F_{\lambda}$-eigenspace of $M_{\ell}$. Since $\rho_{f, \ell}$ is realized on $M_{\ell}$ and since $F$ commutes with the Galois action on $M_{\ell}$ we obtain an exact sequence of $F_{\lambda}$-modules

$$
0 \rightarrow V_{1} \rightarrow M_{\lambda} \rightarrow V_{2} \rightarrow 0
$$

where the $V_{i}$ have dimension $[E: F]$ over $F_{\lambda}$. Choose a number field $K$ such that all endomorphisms in $X$ are defined over $K$ and set $H=G_{p} \cap \operatorname{Gal}(\overline{\mathbb{Q}} / K)$. Then the action of $X$ on $M_{\ell}$ commutes with the action of $H$. In particular the action of $X_{\lambda}=X \otimes_{F} F_{\lambda}$ on $M_{\lambda}$ commutes with the action of $H$. But $H$ acts by distinct characters on the $V_{i}$ by (3.4.7). This shows that $X_{\lambda}$ preserves the spaces $V_{i}$ and therefore for the usual dimension reasons $X_{\lambda}$ must be a matrix algebra over $F_{\lambda}$.

We have shown that $X$ is trivial at all the finite places of $F$. By Theorem 3.1.1 $X$ is trivial at infinity as well and thus $X$ is a matrix algebra over $F$.

The higher weight analog of Ribet's Theorem 3.4.5 is false if $k>2$ and $k$ is odd (see Example 4.3.4 at the end of the paper). However something interesting can still be said in this case. We have:

Theorem 3.4.8. Suppose that $N_{p}=1$ and $C_{p}=0$. If $k \geq 3$ is odd then $X$ is unramified everywhere except possibly at the primes of $F$ lying above $p$ and $\infty$.

Proof. This follows from the fact that the $\ell$-adic part of the proof of Theorem 3.4.6 did not use the fact that $k$ is even.

Corollary 3.4.9. Suppose that $k \geq 3$ is odd and that $f$ is Steinberg at two distinct primes in the level. Then $[F: \mathbb{Q}]$ is even and $X$ is ramified exactly at the infinite places of $F$.

As a final comment let us point out that we have no results in the third case when $N_{p} \geq 2$ and $N_{p} \neq C_{p}$ (except when such a case arises from the previous cases by twisting, in which case one may reduce to the previous cases).

\section{The Case of Quadratic Twisting}

Ribet has remarked [Rib81, page 271] that the cocycle $c$ masks quite effectively whether the Brauer class of $X$ is cohomologically trivial or non-trivial. In this section we compute $c$ explicitly in terms of symbols under the additional assumption that

$$
\chi_{\gamma} \text { is a quadratic character for all non-trivial } \gamma \in \Gamma \text {. }
$$

This formula allows us to give a simple criterion for the structure of $X_{v}$ at each good place $v \mid p$ in terms of the parity of a certain integer $m_{v}$ which is essentially the 
$v$-adic valuation of $a_{p}$ if $a_{p} \neq 0$. If $a_{p}=0$ then a similar result holds if we replace $a_{p}$ by any non-zero Fourier coefficient $a_{p^{\dagger}}$ where $p^{\dagger}$ is a prime satisfying $p p^{\dagger}=1$ $\bmod N$. Before we begin we prove the following lemma.

Lemma 4.0.11. The cusp form $f$ satisfies (4.0.10) if and only if its nebentypus $\epsilon$ is real.

Proof. Let $Q \subset \Gamma$ denote the subgroup of all $\gamma \in \Gamma$ such that $\chi_{\gamma}^{2}=1$. The relation $\chi_{\gamma}^{2}=\epsilon^{\gamma-1}$ shows that $Q=\operatorname{Gal}(E / F(\epsilon))$. Thus $Q=\Gamma$ if and only if $F(\epsilon)=F$ and this is the same as requiring $\epsilon$ to be real since $F$ is totally real.

Let $\mathcal{Q}$ denote the class of primitive non-CM forms $f$ satisfying (4.0.10). By the lemma $\mathcal{Q}$, the class of cusp forms we consider in this section, is exactly the set of primitive non-CM forms with trivial or quadratic nebentypus character.

4.1. Symbols. Let $D$ denote an arbitrary fundamental quadratic discriminant. For a prime $p$ dividing $D$ let $p^{*}$ denote the corresponding prime fundamental discriminant. Thus $q^{*}=(-1)^{(q-1) / 2} \cdot q$ if $q$ is odd and

$$
2^{*}=\left\{\begin{aligned}
1 & \text { if } D \equiv 1 \bmod 4 \\
-4 & \text { if } D \equiv 0 \bmod 4 \text { and } d \equiv 3 \bmod 4 \\
8 & \text { if } D \equiv 0 \bmod 4 \text { and } d / 2 \equiv 1 \bmod 4 \\
-8 & \text { if } D \equiv 0 \bmod 4 \text { and } d / 2 \equiv 3 \bmod 4
\end{aligned}\right.
$$

with $d=D / 4$ when $D \equiv 0 \bmod 4$. It is a fact that every fundamental discriminant $D$ can be uniquely written as a product of prime fundamental discriminants. If $\chi_{D}$ is the corresponding primitive quadratic character of conductor $|D|$ then an analogous decomposition holds for $\chi_{D}$.

Now suppose that $f$ has an extra twist by $\left(\gamma, \chi_{\gamma}\right)$. After (4.0.10) we have $\chi_{\gamma}=$ $\chi_{D}$ for a fundamental discriminant $D=D_{\gamma}$.

Let $q$ be an odd prime. Consider the homomorphism of $\Gamma$ into $\mathbb{Z} / 2$ which maps $\gamma$ to 1 if $q^{*}$ is a factor of $D_{\gamma}$ and maps $\gamma$ to 0 otherwise. Let $\Gamma(q)$ be the kernel of this homomorphism.

Now assume that $q=2$. Let $2_{\gamma}^{*}$ denote the $2^{*}$-part of $D_{\gamma}$ for $\gamma \in \Gamma$. Note that $2_{\gamma}^{*}$ can be uniquely specified by specifying an element in $\mathbb{Z} / 2 \times \mathbb{Z} / 2$ as follows. The two generators $(1,0)$ and $(0,1)$ correspond to the cases $2_{\gamma}^{*}=8$ and $2_{\gamma}^{*}=-8$. The element $(1,1)$ corresponds to $2_{\gamma}^{*}=-4$ and the trivial element corresponds to $2_{\gamma}^{*}=1$. With these conventions the map $\phi_{2}: \Gamma \rightarrow \mathbb{Z} / 2 \times \mathbb{Z} / 2$ defined by $\gamma \mapsto 2_{\gamma}^{*}$ is a homomorphism. Let $\Gamma(2)$ denote the kernel of the homomorphism from $\Gamma$ to $\mathbb{Z} / 2$ obtained by projecting $\phi_{2}$ to the first factor. Similarly let $\Gamma(-2)$ denote the kernel of the homomorphism obtained by projecting $\phi_{2}$ to the second factor.

Note that the subgroups $\Gamma(q)$ for $q$ odd and $\Gamma( \pm 2)$ are all of index one or two in $\Gamma$. Further if $\Gamma(q)$, respectively one of $\Gamma( \pm 2)$, is of index 2 , then necessarily $q \mid N$, respectively $2 \mid N$.

Let $F(q)$ denote the fixed field of $\Gamma(q)$ if $q$ is odd and let $F( \pm 2)$ denote the fixed fields of $\Gamma( \pm 2)$. These fields are at most quadratic extensions of $F$ and can therefore be generated over $F$ by adjoining square roots of elements in $F$, say, $z_{q} \in F$, respectively $z_{ \pm 2} \in F$. 
More explicitly, when $q$ is odd, one may take $z_{q}=a_{n_{q}}^{2}$ where $n_{q}$ is a positive integer satisfying $a_{n_{q}} \neq 0$ and

$$
\chi_{\gamma}\left(n_{q}\right)=\left\{\begin{aligned}
-1 & \text { if } q^{*} \text { occurs in } D_{\gamma} \\
1 & \text { otherwise }
\end{aligned}\right.
$$

for all $\gamma \in \Gamma$. Similarly we may choose $z_{ \pm 2}=a_{n_{ \pm 2}}^{2}$ where $n_{ \pm 2}$ is a positive integer satisfying $a_{n_{ \pm 2}} \neq 0$ and

$$
\chi_{\gamma}\left(n_{ \pm 2}\right)=\left\{\begin{aligned}
-1 & \text { if } 2_{\gamma}^{*}= \pm 8 \text { or }-4 \\
1 & \text { otherwise }
\end{aligned}\right.
$$

for all $\gamma \in \Gamma$.

We are now almost ready to state our result. As motivation and to introduce some more notation recall the following (very special case of a) result of MerkuryevSuslin. Let $K_{2}(F)$ denote the second $K$-group of $F$. Then

$$
\frac{K_{2}(F)}{2 K_{2}(F)}=\operatorname{Br}(F)[2] \text {. }
$$

In particular every 2-torsion element in the Brauer group of $F$ is a product of symbols of the form $(a, b)$ for $a, b \in F$. Here $(a, b)$ is the central simple quaternion algebra over $F$ generated by $1, U, V, U V$, subject to the relations

$$
U^{2}=a, V^{2}=b \text { and } U V=-V U \text {. }
$$

We can now state the following theorem.

Theorem 4.1.3. Let $f \in \mathcal{Q}$. Let $S(N)$ be the set containing all the odd primes dividing $N$ as well as the formal symbols \pm 2 . Then

$$
X=\bigotimes_{q \in S(N)}\left(z_{q}, q^{*}\right)
$$

up to Brauer equivalence, where $q^{*}=(-1)^{\frac{q-1}{2}} q$ if $q$ is odd and $q^{*}= \pm 2$ if $q= \pm 2$.

Proof. The proof is by comparing cocycles on both sides.

Let $D$ denote a fundamental quadratic discriminant. There is a well known formula for the Gauss sum of $\chi_{D}$ given by

$$
G\left(\chi_{D}\right)=\sqrt{\chi_{D}(-1)|D|} .
$$

Thus for $\gamma, \delta$ in $\Gamma$ we have

$$
c(\gamma, \delta)=\frac{G\left(\chi_{\gamma}\right) G\left(\chi_{\delta}\right)}{G\left(\chi_{\gamma \delta}\right)}=\frac{\operatorname{gcd}\left(D_{\gamma}, D_{\delta}\right)}{2^{a}}
$$

where we take the gcd to be negative exactly when both $D_{\gamma}$ and $D_{\delta}$ are negative and where $a=0$ or 1 as follows:

$$
a= \begin{cases}0 & \text { if either } 2_{\gamma}^{*}=1 \text { or } 2_{\delta}^{*}=1 \text { or if } 2_{\gamma}^{*}=2_{\delta}^{*}, \\ 1 & \text { otherwise. }\end{cases}
$$

On the other hand suppose that $q$ is an odd prime dividing $N$. Let $c_{q}$ denote the cocycle on the Galois group $\operatorname{Gal}(F(q) / F)$ corresponding to the symbol $\left(z_{q},\left|q^{*}\right|\right)$. When $F(q)=F$ the class of $c_{q}=1$. When $[F(q): F]=2$ let $\sigma_{q}$ be a generator for $\operatorname{Gal}(F(q) / F)$. Then the cocycle table of $c_{q}$ is 


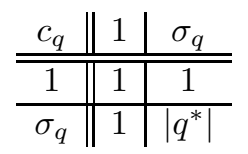

Via inflation

$$
\text { Inf : } \operatorname{Br}(F(q) / F) \hookrightarrow \operatorname{Br}(E / F)
$$

we may consider $c_{q} \in \mathrm{H}^{2}\left(\Gamma, E^{\times}\right)$. We obtain

$$
c_{q}(\gamma, \delta)= \begin{cases}\left|q^{*}\right| & \text { if } p \mid \operatorname{gcd}\left(D_{\gamma}, D_{\delta}\right), \\ 1 & \text { otherwise }\end{cases}
$$

for $\gamma, \delta \in \Gamma$.

Now suppose $q=2$. Let $c_{ \pm 2}$ denote the cocycle on $\operatorname{Gal}(F( \pm 2) / F)$ corresponding to the symbol $\left(z_{ \pm 2}, 2\right)$. When $F( \pm 2)=F$ the class of $c_{ \pm 2}=1$. When $[F( \pm 2)$ : $F]=2$ let $\sigma_{ \pm 2}$ be a generator for $\operatorname{Gal}(F( \pm 2) / F)$. Then the cocycle table of $c_{ \pm 2}$ is

\begin{tabular}{c||c|c}
$c_{ \pm 2}$ & 1 & $\sigma_{ \pm 2}$ \\
\hline \hline 1 & 1 & 1 \\
\hline$\sigma_{ \pm 2}$ & 1 & 2
\end{tabular}

Via inflation

$$
\text { Inf : } \operatorname{Br}(F( \pm 2) / F) \hookrightarrow \operatorname{Br}(E / F)
$$

we may consider $c_{ \pm 2} \in \mathrm{H}^{2}\left(\Gamma, E^{\times}\right)$. As such

$$
c_{ \pm 2}(\gamma, \delta)= \begin{cases}2 & \text { if } 2_{\gamma}^{*}= \pm 8 \text { or }-4, \text { and }, 2_{\delta}^{*}= \pm 8 \text { or }-4 \\ 1 & \text { otherwise }\end{cases}
$$

for $\gamma, \delta \in \Gamma$.

Continuing with $q=2$, consider the homomorphism $s: \mathbb{Z} / 2 \times \mathbb{Z} / 2 \rightarrow \mathbb{Z} / 2$ defined by $s(x, y)=x+y$. Let $\Gamma(-4)$ denote the kernel of the homomorphism $\Gamma \rightarrow \mathbb{Z} / 2$ obtained by composing $s$ with $\phi_{2}: \Gamma \rightarrow \mathbb{Z} / 2 \times \mathbb{Z} / 2$. Then $\Gamma(-4)$ is a subgroup of index 1 or 2 in $\Gamma$. Define $c_{-4}$ to be trivial if $\Gamma(-4)=\Gamma$. Otherwise let $F(-4)$ denote the fixed field of $\Gamma(-4)$, say $F(-4)=F\left(\sqrt{z_{-4}}\right)$ for some $z_{-4} \in F$, and let $c_{-4}$ denote the cocycle corresponding to the symbol $\left(z_{-4}, 4\right)$. Let $\sigma_{-4}$ be a generator for $\operatorname{Gal}(F(-4) / F)$. Then the cocycle table of $c_{-4}$ is

\begin{tabular}{c||c|c}
$c_{-4}$ & 1 & $\sigma_{-4}$ \\
\hline \hline 1 & 1 & 1 \\
\hline$\sigma_{-4}$ & 1 & 4
\end{tabular}

Via inflation

$$
\text { Inf : } \operatorname{Br}(F(-4) / F) \hookrightarrow \operatorname{Br}(E / F)
$$

we may consider $c_{-4} \in \mathrm{H}^{2}\left(\Gamma, E^{\times}\right)$. As such

$$
c_{-4}(\gamma, \delta)= \begin{cases}4 & \text { if } 2_{\gamma}^{*}, 2_{\delta}^{*} \in\{ \pm 8\} \\ 1 & \text { otherwise }\end{cases}
$$

for $\gamma, \delta \in \Gamma$.

Finally let $\Gamma(\infty)$ denote the subgroup of $\gamma \in \Gamma$ such that $D_{\gamma}$ is positive. Again $\Gamma(\infty)=\Gamma$ or $\Gamma(\infty)$ is an index two subgroup of $\Gamma$. In the former case we set $c_{\infty}$ to be the trivial cocycle on $\Gamma$. In the latter case we define $c_{\infty}$ as follows. Let $F(\infty)$ 
denote the fixed field of $\Gamma(\infty)$. Then $F(\infty)$ is a quadratic extension of $F$. In fact $F(\infty)=F\left(\sqrt{z_{\infty}}\right)$ where

$$
z_{\infty}=z_{-2} \times \prod_{q^{*}<0} z_{q}
$$

where the product on $q$ is over odd primes $q$.

This is because if $\gamma \in \Gamma(\infty)$ then $D_{\gamma}$ is divisible either by an even number of $q^{*}$ with $q^{*}<0$ and $p$ odd, or, by an odd number of $q^{*}$ with $q^{*}<0, p$ odd, and $2_{\gamma}^{*}=-8$ or -4 . In either case $\sqrt{z_{\infty}}=\sqrt{z_{-2}} \times \prod_{q^{*}<0} \sqrt{z_{q}}$ is fixed by $\gamma$. Now let $\sigma_{\infty}$ denote a generator of $\operatorname{Gal}(F(\infty) / F)$. Let $c_{\infty}$ denote the cocycle on $\operatorname{Gal}(F(\infty) / F)$ corresponding to the symbol $\left(z_{\infty},-1\right)$. Its cocycle table is given by

$$
\begin{array}{c||c|c}
c_{\infty} & 1 & \sigma_{\infty} \\
\hline \hline 1 & 1 & 1 \\
\hline \sigma_{\infty} & 1 & -1
\end{array}
$$

We note that (4.1.4) and a standard bilinearity property of symbols imply that

$$
\left(z_{\infty},-1\right)=\left(z_{-2},-1\right) \otimes \prod_{q^{*}<0}\left(z_{q},-1\right)
$$

in the Brauer group. As usual we may think of $c_{\infty}$ as a cocycle on $\Gamma$ by inflation. As such $c_{\infty}(\gamma, \delta)=1$ except when both $D_{\gamma}$ and $D_{\delta}$ are negative, in which case $c_{\infty}(\gamma, \delta)=-1$.

With the above definitions one may now compare cocycles on both sides. One may check that

$$
c=c_{\infty} \cdot c_{2} \cdot c_{-2} \cdot c_{-4} \cdot \prod_{q \mid N} c_{q}
$$

as cocycles on $\Gamma$. Thus

$$
\begin{aligned}
X & =\left(z_{\infty},-1\right) \otimes\left(z_{2}, 2\right) \otimes\left(z_{-2}, 2\right) \otimes\left(z_{-4}, 4\right) \otimes \bigotimes_{\substack{q \mid N \\
q \text { odd }}}\left(z_{q},\left|q^{*}\right|\right) \\
& =\left(z_{2}, 2\right) \otimes\left(z_{-2},-2\right) \otimes\left(z_{-4}, 4\right) \otimes \bigotimes_{\substack{q \mid N \\
q \text { odd }}}\left(z_{q}, q^{*}\right) \\
& =\left(z_{2}, 2\right) \otimes\left(z_{-2},-2\right) \otimes \bigotimes_{\substack{q \mid N \\
q \text { odd }}}\left(z_{q}, q^{*}\right) .
\end{aligned}
$$

where the second equality follows from (4.1.5) and the last equality follows from fact that $(a, 4)=1$ for any $a \in F^{\times}$. This proves the theorem.

Remark 4.1.6. After the above theorem was proved it was pointed out to us that a similar result was recently proved by Quer in [Que98]. Quer's result is for $k=2$, but does not assume the condition (4.0.10).

We now wish to evaluate the symbols that appear in the expression for $X$ in Theorem 4.1.3 above. First let us recall some general facts about symbols.

Let $F$ be an arbitrary number field. Let $v$ denote a place of $F$ which is either finite or infinite. Let $F_{v}$ denote the completion of $F$ at $v$. It is well known that

$$
\operatorname{Br}\left(F_{v}\right) \cong \mathbb{Q} / \mathbb{Z}
$$


if $v$ is finite (and is $\mathbb{Z} / 2$ if $v$ is infinite and real and is trivial if $v$ is infinite and complex).

Now let $a$ and $b$ be non-zero elements of $F$. Then the symbol $(a, b)$ determines an element in $\operatorname{Br}(F)[2]$. For each finite place $v$ of $F$, let $(a, b)_{v}$ denote the induced element of $\operatorname{Br}\left(F_{v}\right)[2]$. By (4.1.7) the symbol $(a, b)_{v}$ is completely specified by a sign +1 or -1 . This sign can be computed in terms of the $v$-adic valuations of $a$ and $b$. There are two cases, the tame case: $v \nmid 2$, and the wild case: $v \mid 2$.

First assume that $v$ is prime to 2. Fix a uniformizer $\pi_{v}$ of the ring of integers of $F_{v}$. Write

$$
\begin{aligned}
a & =\pi_{v}^{v(a)} \cdot a^{\prime} \\
b & =\pi_{v}^{v(b)} \cdot b^{\prime}
\end{aligned}
$$

where we consider $v$ here to be normalized such that $v\left(\pi_{v}\right)=1$. In this section $v$ will refer to a valuation which is normalized in this way unless explicitly stated otherwise. Then one has

$$
(a, b)_{v}=(-1)^{\frac{N v-1}{2}} v(a) v(b) \cdot\left(\frac{b^{\prime}}{v}\right)^{v(a)} \cdot\left(\frac{a^{\prime}}{v}\right)^{v(b)} .
$$

Here the symbol $\left(\frac{c}{v}\right)$ takes the values \pm 1 and is 1 exactly when the image of $c$ is a square in the residue field at $v$.

Now assume that $v \mid 2$. We shall only treat the case $F=\mathbb{Q}$ so that $v=2$. For a unit $u \in \mathbb{Q}_{2}^{\times}$let $\varepsilon(u)$ denote the residue of $(u-1) / 2$ in $\mathbb{Z} / 2$ and let $\omega(u)$ denote the residue of $\left(u^{2}-1\right) / 8$ in $\mathbb{Z} / 2$. Then for units $u, v$ in $\mathbb{Q}_{2}^{\times}$we have

$$
\begin{aligned}
& (u, v)_{2}=(-1)^{\varepsilon(u) \varepsilon(v)}, \\
& (2, u)_{2}=(-1)^{\omega(u)} .
\end{aligned}
$$

Note that these formulas completely determine $(a, b)_{2}$ for $a, b \in \mathbb{Q}_{2}^{\times}$.

Now let us return to our situation. Let $F$ be the number field generated by the numbers $a_{p}^{2}$ for $p \nmid N$ (we can drop the $\epsilon(p)^{-1}$ term since it is just \pm 1 ). The exact sequence (3.0.6) shows that the Brauer class of $X$ is completely determined by the Brauer classes of the $X_{v}$, which are in turn completely determined by specifying a $\operatorname{sign}$, one for each $v$. For notational convenience we write $X_{v} \sim a$ for an integer $a$ if the sign of the Brauer class of $X_{v}$ is the same as $(-1)^{a}$.

Recall that $X_{v} \sim k$ if $v$ is infinite. Moreover for a good place $v$ of $F$ we have $X_{v} \sim 0$ if $v$ is ordinary for $f$ (cf. Theorem 3.3.1) or $v$ is singular and the denominator of the valuation of $a_{p}$ is odd (cf. Theorem 3.3.2). The following theorem generalizes these results (for the cusp forms considered in this section). To state it we introduce a positive integer $m_{v}$ for each good place $v$ of $F$ of residue characteristic $p$ with $a_{p} \neq 0$ :

$$
m_{v}:=\left[F_{v}: \mathbb{Q}_{p}\right] \cdot v\left(a_{p}^{2}\right)
$$

In the definition of $m_{v}$ we take the valuation $v$ which is normalized such that $v(p)=1$. Then we have:

Theorem 4.1.11. Let $p$ be a prime such that $p \nmid N$ and $a_{p} \neq 0$. Let $v$ be a place of $F$ lying over $p$. If $p \neq 2$ we have

$$
X_{v} \sim \begin{cases}0 & \text { if }\left(\frac{q^{*}}{p}\right)=1 \text { for all } q \in S(N), \\ m_{v} & \text { otherwise. }\end{cases}
$$


If $p=2$ then the same conclusion holds if $F=\mathbb{Q}$.

Proof. By Theorem 4.1.3 we have

$$
X_{v}=\bigotimes_{q \in S(N)}\left(z_{q}, q^{*}\right)_{v} .
$$

Since $v$ is good, that is since $v$ is prime to $N$, we have $v\left(q^{*}\right)=0$. First assume that $p \neq 2$. Then $v$ is prime to 2 so that by (4.1.8) we have $\left(z_{q}, q^{*}\right)_{v}=\left(\frac{q^{*}}{v}\right)^{v\left(z_{q}\right)}$. But $\left(\frac{q^{*}}{v}\right)=\left(\frac{q^{*}}{p}\right)^{f_{v}}$ since every element of $\mathbb{F}_{p}$ has a square root over a quadratic extension of $\mathbb{F}_{p}$. We conclude that

$$
\left(z_{q}, q^{*}\right)_{v}=\left(\frac{q^{*}}{p}\right)^{f_{v} \cdot v\left(z_{q}\right)} .
$$

Thus if $\left(\frac{q^{*}}{p}\right)=1$ for all $q \in S(N)$ we have $X_{v}=1$, as desired.

Suppose on the other hand that the subset $S^{-}$of elements of $S(N)$ such that $\left(\frac{q^{*}}{p}\right)=-1$ is non-empty. Write the primes in $S^{-}$as $q_{1}, q_{2}, \ldots q_{m}$ with $m \geq 1$. As usual we allow one or both of \pm 2 to be in this list. Define distinct primes $r_{j}$ for $j=0,1, \ldots, r_{m-1}$ as follows: set $r_{0}=p$ and define $r_{j}$ for $j=1, \ldots, m-1$ recursively by

$$
\begin{aligned}
& \left(\frac{q_{i}^{*}}{r_{j}}\right)=(-1)^{\delta_{i j}} \cdot\left(\frac{q_{i}^{*}}{r_{j-1}}\right) \text { for all } i=1, \ldots, m, \text { and, } \\
& \left(\frac{q^{*}}{r_{j}}\right)=1 \text { if } q \in S(N) \backslash S^{-} .
\end{aligned}
$$

We may and do assume that each $a_{r_{j}} \neq 0$. This can be done for $j=0$ since $a_{p} \neq 0$ by hypothesis. For the other $r_{j}$ 's we simply note that if $a_{r_{j}}=0$ for all $r_{j}$ defined by the congruence conditions (4.1.13) and (4.1.14) then the set of primes $p$ for which $a_{p}=0$ would have a positive density contradicting a result of Serre which says that for non-CM forms the density of such primes is 0 [Ser81, Theorem 15].

Recall that for a prime $q \in S(N), z_{q}=a_{n_{q}}^{2} \in F$ where $n_{q}$ is a positive integer determined by the congruence conditions (4.1.1) and (4.1.2) and the condition $a_{n_{q}} \neq$ 0 . It is only the primes $q \in S^{-}$that contribute to the sign of $X_{v}$ since if $q \notin S^{-}$ then $\left(\frac{q^{*}}{v}\right)=\left(\frac{q^{*}}{p}\right)=1$. If $q \in S^{-}$then $q=q_{i}$ for some $i$ with $1 \leq i \leq m$. Set

$$
n_{q_{i}}= \begin{cases}r_{i-1} \cdot r_{i} & \text { if } 1 \leq i \leq m-1, \\ r_{m-1} & \text { if } i=m\end{cases}
$$

Clearly $a_{n_{q_{i}}} \neq 0$ since on distinct primes the Fourier coefficients are multiplicative, and the $r_{j}$ were chosen so that each $a_{r_{j}} \neq 0$. Furthermore the $n_{q_{i}}$ satisfy the congruence conditions (4.1.1) and (4.1.2). Indeed suppose that $q_{i}^{*}$ occurs in $D_{\gamma}$ (when $q_{i}= \pm 2$ we mean as usual that $2_{\gamma}^{*}= \pm 8$ or -4 ). Then if $i<m$

$$
\chi_{\gamma}\left(n_{q_{i}}\right)=\chi_{\gamma}\left(r_{i-1}\right) \chi_{\gamma}\left(r_{i}\right)=-1
$$

since, by (4.1.13) and (4.1.14), $\chi_{\gamma}\left(r_{i-1}\right)$ and $\chi_{\gamma}\left(r_{i}\right)$ differ by a sign. Similarly $\chi_{\gamma}\left(r_{i-1}\right)=\chi_{\gamma}\left(r_{i}\right)$ if $q_{i}^{*}$ does not occur in $D_{\gamma}$ so that $\chi_{\gamma}\left(n_{q_{i}}\right)=1$ in this case. If 
$i=m$ then

$$
\chi_{\gamma}\left(n_{q_{m}}\right)=\chi_{\gamma}\left(r_{m-1}\right)=\prod_{q_{i}^{*} \text { in } D_{\gamma}}\left(\frac{q_{i}^{*}}{r_{m-1}}\right) .
$$

But (4.1.13) shows that

$$
\left(\frac{q_{i}^{*}}{r_{m-1}}\right)=\left\{\begin{array}{rlrl}
-\left(\frac{q_{i}^{*}}{p}\right) & =1 & & \text { if } i \leq m-1, \\
\left(\frac{q_{i}^{*}}{p}\right)=-1 & & \text { if } i=m .
\end{array}\right.
$$

So $\chi_{\gamma}\left(n_{q_{m}}\right)=-1$ if and only if $q_{m}$ occurs in $D_{\gamma}$ as desired.

Now if $q_{i} \in S^{-}$we have $z_{q_{i}}=a_{n_{q_{i}}}^{2}$ and $\left(\frac{q_{i}^{*}}{p}\right)=-1$ so that by (4.1.12) we have

$$
\left(z_{q_{i}}, q_{i}^{*}\right)_{v} \sim f_{v} \cdot v\left(a_{n_{q_{i}}}^{2}\right) .
$$

Substituting for $n_{q_{i}}$ from (4.1.15) above and multiplying over all $i$ in $\{1, \ldots, m\}$, there is a telescoping effect, the result of which is

$$
X_{v} \sim f_{v} \cdot v\left(a_{p}^{2}\right)
$$

If we take the $v(p)=1$ normalization for $v$ then the right hand side becomes $m_{v}$, proving the theorem in the case $p \neq 2$.

Now assume that $p=2$ and that $F=\mathbb{Q}$. Write $v_{2}\left(z_{q}\right)$ for the power of 2 that divides $z_{q}$ and define $z_{q}^{\prime}$ by $z_{q}=2^{v_{2}\left(z_{q}\right)} \cdot z_{q}^{\prime}$. We have

$$
\left(z_{q}, q^{*}\right)_{2}=\left(2, q^{*}\right)_{2}^{v_{2}\left(z_{q}\right)} \cdot\left(z_{q}^{\prime}, q^{*}\right)_{2} \text {. }
$$

One can easily check that $\left(2, q^{*}\right)_{2}$ is equal to $(-1)^{\omega\left(q^{*}\right)}$ by $(4.1 .10)$ which may again be easily checked to be the same as $\left(\frac{q^{*}}{2}\right)$ using the fact 2 splits in $\mathbb{Q}\left(\sqrt{q^{*}}\right)$ if and only if $q^{*} \equiv 1 \bmod 8$. On the other hand $\left(z_{q}^{\prime}, q^{*}\right)_{2}=1$ by $(4.1 .9)$ since $\varepsilon\left(q^{*}\right) \equiv 0$ $\bmod 2$. Thus

$$
\left(z_{q}, q^{*}\right)_{2}=\left(\frac{q^{*}}{2}\right)^{v_{2}\left(z_{q}\right)} .
$$

Now the argument proceeds as in the case $p \neq 2$ proving the theorem in this case as well.

Remark 4.1.16. The assumption that $F=\mathbb{Q}$ when $p=2$ could probably be removed if one had formulas for wild symbols other than in the case $F=\mathbb{Q}$.

4.2. The case $a_{p}=0$. We now treat the case $a_{p}=0$ (and $v$ is good). Clearly the structure of $X_{v}$ in this case is not determined by the parity of $m_{v}$ since $m_{v}=$ $\infty$. However as we now show the structure of $X_{v}$ is still determined by a Fourier coefficient at a prime $p^{\dagger}$, closely related to $p$.

In fact we take $p^{\dagger}$ to be any prime such that $p p^{\dagger} \equiv 1 \bmod N$ and such that $a_{p^{\dagger}} \neq 0$. Serre's result, quoted above, implies that one can always find such a $p^{\dagger}$. Set

$$
m_{v}^{\dagger}:=\left[F_{v}: \mathbb{Q}_{p}\right] \cdot v\left(a_{p^{\dagger}}^{2}\right) .
$$

Theorem 4.1.11 now has the following avatar when $a_{p}=0$. 
Proposition 4.2.1. Let $v$ be a place of $F$ of residue characteristic p prime to $N$ and say $a_{p}=0$. Let $m_{v}^{\dagger}$ be as above. If $p \neq 2$ we have

$$
X_{v} \sim \begin{cases}0 & \text { if }\left(\frac{q^{*}}{p^{\dagger}}\right)=1 \text { for all } q \in S(N), \\ m_{v}^{\dagger} & \text { otherwise. }\end{cases}
$$

If $p=2$ then the same conclusion holds if $F=\mathbb{Q}$.

Proof. Since $p p^{\dagger} \equiv 1 \bmod N$ we have

$$
\left(\frac{q^{*}}{p}\right)=\left(\frac{q^{*}}{p^{\dagger}}\right)
$$

so that the proof of Theorem 4.1.11 goes through replacing $p$ with $p^{\dagger}$.

Let us record the following easy consequences of the above results which are valid for the cusp forms considered in this section, namely those satisfying (4.0.10). The first result is a stronger version of Corollary 3.3.3 (away from $p=2$ ).

Corollary 4.2.2. Let $v$ be a place of $F$ of residue characteristic $p$ with $p \nmid 2 N$. If $p$ is unramified in $E$ then $X_{v}$ is a matrix algebra over $F_{v}$.

Proof. This is immediate from Theorem 4.1.11 and Proposition 4.2.1 since in this case the integer $m_{v}$ or $m_{v}^{\dagger}$ is necessarily even.

Corollary 4.2.3. If $X$ is ramified at $v$ then $v$ must divide either the discriminant of the field $E$, or $2 N$, or $\infty$.

4.3. Numerical examples. We now give some examples to illustrate the theorems proved in this paper. These computations were done using the $\mathrm{C}++$ program Endohecke, written by the authors, by suitably modifying and extending parts of W. Stein's modular forms package Hecke. The algorithm is based on the explicit formula for $X$ in Theorem 4.1.3. In fact Endohecke only computes the image of $X$ under the norm map from $\operatorname{Br}(F)$ to $\operatorname{Br}(\mathbb{Q})$. In particular it computes $X$ completely only when $F=\mathbb{Q}$.

In all the examples below $f$ is a non-CM primitive form. In the first four examples $F=\mathbb{Q}$ and we give the complete structure of $X$ as an element of the Brauer group of $\mathbb{Q}$. The fifth and last example discusses a case where $F \neq \mathbb{Q}$.

Example 4.3.1. Let $f \in S_{2}(512,1)$ be the unique primitive form of orbit size 4 . This example was considered in [Ste00]. It turns out that $f$ has extra twists by $\chi_{1}, \chi_{ \pm 8}$ and $\chi_{-4}$ and $X$ is ramified at 2 and 3 . No theorem of ours predicts the ramification at the bad place 2 , but at the good place 3 the ramification is predicted by Theorem 4.1 .11 since $a_{3}^{2}=6$ has odd 3 -adic valuation.

Example 4.3.2. Let $f \in S_{2}\left(57, \chi_{57}\right)$ be the unique primitive form of orbit size 4 . Then $f$ has extra twists by $\chi_{0}, \chi_{-3}, \chi_{-19}$ and $\chi_{57}$ and $X$ is ramified at 2 and 5 . This is as predicted by Theorem 4.1.11 since $a_{2}^{2}=2$ and $a_{5}^{2}=-5$. Note that at the bad places, namely 3 and $19, A$ has potentially good ordinary reduction in the sense of Theorem 3.4.1, so that $X$ is split at these places.

Example 4.3.3. Let $f \in S_{2}\left(469, \chi_{469}\right)$ be the unique primitive form of orbit size 4 . Then $f$ has extra twists by $\chi_{1}, \chi_{-7}, \chi_{-67}$ and $\chi_{469}$ and $X$ is ramified at 2 and 5 . The ramification at 2 is predicted by Theorem 4.1 .11 since $a_{2}^{2}=-2$. On the other hand $a_{5}=0$ so we must turn to Proposition 4.2.1. We may choose $5^{\dagger}=563$. Since $a_{563}^{2}=810=2 \cdot 3^{4} \cdot 5$ has 5 -adic valuation 1 , we see that $X$ is ramified at 5 . 
Example 4.3.4. Let $f \in S_{3}\left(38, \chi_{-19}\right)$ be the unique primitive form of orbit size 2. Then $f$ has extra twists by $\chi_{1}, \chi_{-19}$ and $X$ is ramified at 2 and $\infty$. The ramification at 2 shows that the higher weight analog of Theorem 4.3.4 is false in odd weight. The ramification at $\infty$ is predicted by Momose's Theorem 3.1.1. To illustrate Theorem 3.3.1 consider $p=5$. Since $a_{5}^{2}=-8$, we see that $f$ is ordinary at 5 and therefore $X$ is split at 5 .

Example 4.3.5. Let $f \in S_{4}\left(57, \chi_{57}\right)$ be the unique primitive form of orbit size 12 . Then $f$ has extra twists by $\chi_{1}, \chi_{-3}, \chi_{-19}$ and $\chi_{57}$. Thus $[F: \mathbb{Q}]=3$. Our program shows that $X$ is ramified at an odd number of primes lying over 3 and 101 and an even number of primes (possibly zero) lying above 2 .

We illustrate Theorems 3.3.2 and 3.4.4. For the first take $p=5$. Then 5 is unramified in $F$ and $5=v_{1} v_{2}$ with residue degrees $f_{1}=1, f_{2}=2$ respectively. Let $w_{1}$ be a place of $E$ lying above $v_{1}$. Then $w_{1}\left(a_{5}\right)=1$ which has odd denominator and $2=v_{1}\left(a_{5}^{2}\right)<k-1=3$. Thus Theorem 3.3.2 guarantees that $X$ is split at $v_{1}$.

On the other hand consider the bad prime $p=19$. It turns out 19 is unramified in $F$ and that $19=v_{1} v_{2} v_{3}$ in $F$ with $f_{1}=f_{2}=f_{3}=1$. Moreover for each place $w$ of $E$ lying over, say, $v_{1}$, one checks, by replacing $w$ by $\bar{w}$ if necessary, that $w\left(a_{19}\right)=1$, so that these numbers are equal, have odd denominator, and are smaller than $(k-1) / 2=3 / 2$. Thus $X$ splits at $v_{1}$ by Theorem 3.4.4.

Acknowledgements. We wish to thank Professors K. Ribet and A. Scholl for some useful email correspondence.

\section{REFERENCES}

[AL78] A. O. L. Atkin and Wen Ch'ing Winnie Li. Twists of newforms and pseudo-eigenvalues of $W$-operators. Invent. Math., 48(3):221-243, 1978.

[BR93] D. Blasius and J. Rogawski. Motives for Hilbert modular forms. Invent. Math., 114:5587, 1993.

[Bre01] C. Breuil. Lectures on p-adic Hodge theory, deformations and local Langlands, Volume 20, Advanced course lecture notes. Centre de Recerca Matemàtica, Barcelona.

[Del69] P. Deligne. Formes Modulaires et représentations $\ell$-adiques. In Seminaire Bourbaki, 1968/1969, exposé 355, Lecture Notes in Math. 179, pages 139-172. Springer-Verlag, 1971.

[DR73] P. Deligne and M. Rapoport. Les schémas de modules de courbes elliptique. In Modular functions of one variable, II (Proc. Internat. Summer School, Univ. Antwerp, Antwerp, 1972), volume 349 of Lecture Notes in Math., pages 143-316. Springer, Berlin, 1973.

[Dem72] M. Demazure. Lectures on p-divisible groups, volume 302 of Lecture Notes in Mathematics. Springer-Verlag, 1972.

[Fal83] G. Faltings. Endlichkeitssätze für abelsche Varietäten über Zahlkörpen. Invent. Math., 73:349-366, 1983

[FM83] J.-M. Fontaine and B. Mazur. Geometric Galois representations. In Elliptic curves, modular forms, $8 \mathcal{F}$ Fermat's last theorem. Proceedings ot the conference on elliptic curves and modular forms held at the Chinese University of Hong Kong, December 18-21, 1993. International Press. Ser. Number Theory. 1, 41-78 (1995).

[Hid00] H. Hida. Modular Forms and Galois Cohomology. Cambridge University Press, Cambridge, 2000.

[Jan92] U. Jannsen. Motives, numerical equivalence, and semi-simplicity. Invent. Math., 107(3):447-452, 1992.

[Mom81] F. Momose. On the $l$-adic representations attached to modular forms. J. Fac. Sci. Univ. Tokyo Sect. IA Math., 28:89-109, 1981.

[Que98] J. Quer. La classe de Brauer de l'algèbre d'endomorphismes d'une variété abélienne modulaire. C. R. Acad. Sci. Paris Sér. I Math., 327(3):227-230, 1998.

[Rib80] K. Ribet. Twists of modular forms and endomorphisms of abelian varieties. Math. Ann., 253(1):43-62, 1980. 
[Rib81] K. Ribet. Endomorphism algebras of abelian varieties attached to newforms of weight 2. In Seminar on Number Theory, Paris 1979-80, Progr. Math., 12, pages 263-276. Birkhäuser, Boston, Mass., 1981.

[Rib92] K. Ribet. Abelian varieties over $\mathbb{Q}$ and modular forms. Proc. KAIST Math. Workshop, pages 53-79, 1992.

[Sch90] A. Scholl. Motives for modular forms. Invent. Math., 100:419-430, 1990.

[Ser81] J.-P. Serre. Quelques applications du théorème de densité de Chebotarev. Inst. Hautes Études Sci. Publ. Math., 54:323-401, 1981.

[Shi71] G. Shimura. On elliptic curves with complex multiplication as factors of the Jacobians of modular function fields. Ngoya Math. J., 43:199-208, 1971.

[Shi73] G. Shimura. On the factors of the Jacobian variety of a modular function field. J. Math. Soc. Japan, 25:523-544, 1973.

[Ste00] W. Stein. The first newform such that $\mathbb{Q}\left(a_{n}\right) \neq \mathbb{Q}\left(a_{1}, a_{2}, \ldots\right)$ for all $n$. Preprint, 2000.

[Vol01] M. Volkov. Les représentations $\ell$-adiques associées aux courbes elliptiques sur $\mathbb{Q}_{p} . J$. Reine Angew. Math., 535:65-101, 2001.

School of Mathematics, Tata institute of Fundamental Research, Homi Bhabha RoAd, Mumbai 400 005, India.

E-mail address: abrown@math.tifr.res.in, eghate@math.tifr.res.in 